\title{
VARIEDADES SUB-RIEMANNIANAS DE CONTATO DE DIMENSÃO 3
}

\author{
Marcos Monteiro Diniz
}

\author{
DISSERTAÇÃO APRESENTADA \\ $\mathrm{AO}$ \\ INSTITUTO DE MATEMÁTICA E ESTATÍSTICA \\ DA \\ UNIVERSIDADE DE SÃO PAULO \\ PARA \\ OBTENÇÃO DO GRAU \\ $\mathrm{DE}$ \\ MESTRE EM MATEMÁTICA \\ Área de Concentração: Geometria \\ Orientador: Prof. Dr. José Antônio Verderesi
}

Durante a elaboração deste trabalho, o autor recebeu apoio financeiro da UFPa e CAPES

- São Paulo, setembro de 1996 - 


\section{VARIEDADES SUB-RIEMANNIANAS DE CONTATO DE DIMENSÃO 3}

Este trabalho corresponde à redação final da dissertação devidamente corrigida e apresentada por Marcos Monteiro Diniz e aprovada pela Comissão Julgadora

São Paulo, 23 de setembro de 1996

Banca Examinadora:

- Prof. Dr. José Antônio Verderesi (Orientador) - IME-USP

- Prof. Dr. Cláudio Gorodski - IME-USP

- Prof. Dr. Renato Pedrosa - IMECC-UNICAMP 


\section{RESUMO}

Nesse trabalho, introduzimos a noção de variedades sub-Riemannianas de contato e estudamos o caso de dimensão 3.

Os conceitos de fibrado de círculo, conexão e transporte paralelo são dados e as equações de estrutura, definidas. Provamos a existência e unicidade da "forma de conexão sub-Riemanniana" e apresentamos as Identidades de Bianchi. Seguem então alguns cálculos relacionados aos exemplos clássicos $H^{3}, S^{3}$ e $Q^{3}$.

O teorema de existência e unicidade é apresentado em termos da conexão adaptada $\nabla$ em $M$ e a relação entre $\nabla$ e o transporte paralelo é rapidamente discutido.

Finalmente, definimos variedades homogêneas e provamos o principal resultado - os teoremas de classificação para variedades sub-Riemannianas de contato, homogêneas, simplesmente conexas, de dimensão 3 - fornecendo uma lista dos modelos. 


\section{ABSTRACT}

In this work we introduce the notion of contact sub-Riemannian manifold and study the three-dimensional case.

The concepts of circle bundle, connection and parallel transport are given and the structure equations are defined. We prove the existence and unicity of the sub-Riemannian connection form and present the Bianchi's Identities. It follows some computatations related to the classical examples $H^{3}, S^{3}$ and $Q^{3}$.

The theorem of existence and unicity is presented in terms of the adapted connection $\nabla$ on $M$ and the relation between $\nabla$ and the parallel transport is discussed.

Finally, we define homogeneous manifolds and prove the main result - the classification theorems for homogeneous, simple-connected, contact sub-Riemannian manifold of dimension 3 - giving a list of the models. 
SOMA DE MALOGROS,

NOVES FORA TUDO

Com despedício de cores, selo o fim dos meus amores.

Amor pede ser começo

de si mesmo a cada instante.

Fico no fim que mereço.

Sei que perdi: me apostei inteiro. Mas aprendi que não dependo (e ninguém) só de mim para me dar. É repartido que posso vir um dia a merecer a flama ardendo serena, que resolve a diferença entre viver e morrer.

Sei que perdi. Mas ganhei. 


\section{AGRADECIMENTOS}

Gostaria de agradecer primeiramente ao Prof. José Antônio Verderesi, pela paciência e perfeita orientação dada a mim durante esses dois anos e pouco.

Aos professores do grupo de Geometria Sub-Riemanniana, pela oportunidade que a mim propiciaram de participar dos seminários e de obter um pouco mais de conhecimento acerca da geometria, obrigado.

À Vivi, Bea e Belinha, três amores meus, cujos sorrisos e os carinhos foram (e só eu sei o quanto!) essenciais para mim nesses últimos anos. A elas, todos os meus beijos de gratidão.

Aos meus tios, Daniel e Nazaré, e ao meu amigo/irmão Stéfano pelo carinho e ajuda que me proporcionaram durante o período mais difícil dessa caminhada. Muito obrigado.

Ao Prof. Carlos Knudsen, pela amizade e orientação (inclusive pela indicação do meu orientador) e ao Prof. João Batista do Nascimento, pela amizade e por todas as ajudas e conversas que muito me auxiliaram a esclarecer vários pontos, obrigado.

Ao Zé, companheiro de bola e amigo de vida, cuja compreensão e sensibilidade me surpreenderam e cuja obtenção da amizade é o que mais me dá a certeza de que "ganhei" aqui. Valeu, Zé!

Finalmente a todos os colegas e amigos do IME, com quem pude dividir todos os momentos e compartilhar um pouco de mim mesmo nesses dois anos e meio. Muito obrigado. Muchas gracias. 
À Ana Diniz, que me deu os primeiros exemplos de amor à cultura e à ciência. 


\section{Sumário}

Introdução

1 Variedades sub-Riemannianas de contato 3

1.1 Variedade sub-Riemanniana, forma de contato associada, campo fundamental 3

1.2 Exemplos . . . . . . . . . . . . . . . . . . . 7

2 Conexão sub-Riemanniana $\quad 16$

2.1 Fibrado de círculo, conexão, forma de conexão . . . . . . . . . . . . 17

2.2 Transporte paralelo . . . . . . . . . . . . . . . . . . . . 20

2.3 Equações de estrutura, forma da conexão sub-Riemanniana . . . . . . . . . 23

2.4 As identidades de Bianchi . . . . . . . . . . . . . . . . . . . . . . . . . . . . . . . . 33

2.5 Exemplos . . . . . . . . . . . . . . . . . . 33

$\begin{array}{lll}3 & \text { Derivada covariante } & 38\end{array}$

3.1 Derivada covariante sub-Riemanniana . . . . . . . . . . . . 38

3.2 Relação entre derivada covariante e transporte paralelo . . . . . . . . . . . 46

4 Classificação das variedades homogêneas $\quad \mathbf{5 0}$

4.1 Isometria sub-Riemanniana, variedade homogênea . . . . . . . . . . . . . 50

4.2 Grupos de isometrias de $H^{3}, S^{3}$ e $Q^{3} \ldots \ldots \ldots \ldots \ldots \ldots$

4.3 Teoremas de classificação . . . . . . . . . . . . . . . . . 55

4.4 Classificação das variedades sub-Riemannianas de contato homogêneas de dimensão $3 \ldots \ldots \ldots$. . . . . . . . . . . . . . . . 73

Referências $\quad 82$ 


\section{Introdução}

Uma variedade sub-Riemanniana de contato nada mais é que uma variedade diferenciável, com uma distribuição de contato munida de uma métrica.

Este trabalho tem por objetivo apresentar os conceitos fundamentais da geometria das variedades sub-Riemannianas de contato de dimensão 3 e a classificação das variedades sub-Riemannianas homogêneas, dando ao teorema de classificação, uma demonstração essencialmente geométrica, distinta portanto da fornecida em [5], a qual se dá em termos de álgebra de Lie.

No primeiro capítulo, definimos variedade sub-Riemanniana de contato e forma de contato associada. Demonstramos a existência e unicidade do campo fundamental e, por fim, determinamos a forma de contato e o campo fundamental dos três exemplos clássicos apresentados: o espaço de Heisenberg $H^{3}$, a esfera $S^{3}$ e a quádrica $Q^{3}$.

No capítulo 2, associamos à variedade um $S^{1}$-fibrado canônico e apresentamos a definição de conexão nesse fibrado e de transporte paralelo de vetores tangentes à variedade. Apresentamos então as equações de estrutura, as identidades de Bianchi e demonstramos a existência de uma única conexão, canonicamente associada à variedade, de torsão mínima quando "vista da distribuição", que é essencialmente a definida por S.S.Chern e R.S.Hamilton em [4].

Já no terceiro capítulo, provamos a equivalência entre fornecer uma conexão e dar uma derivada covariante com certas propriedades, o que nos possibilitou reenunciar o teorema de existência e unicidade da conexão sub-Riemanniana em termos da derivada covarian- 
te. Ainda nesse capítulo, foi brevemente discutida a relação entre derivada covariante e transporte paralelo.

No quarto e último capítulo, definimos variedades homogêneas, introduzimos o conceito de isometria entre variedades sub-Riemannianas e demonstramos o resultado principal do trabalho, os teoremas de classificação (4.2 e 4.3) para variedades sub-Riemannianas homogêneas, de contato, simplesmente conexas, de dimensão 3. Terminamos então esse capítulo, fornecendo uma lista dos modelos para essas variedades, obtida a partir da análise de seus invariantes geométricos. 


\section{Capítulo 1}

\section{Variedades sub-Riemannianas de contato}

Introduz-se neste capítulo o conceito de variedades sub-Riemannianas de contato, de forma de contato associada. É demonstrada também a existência e unicidade do campo fundamental. Por fim, seguem três exemplos clássicos de variedades sub-Riemannianas de contato de dimensão 3 , o espaço de Heisenberg $H^{3}$, a esfera $S^{3}$ e a quádrica $Q^{3}$.

\subsection{Variedade sub-Riemanniana, forma de contato as- sociada, campo fundamental.}

Definição 1.1 Seja M uma variedade diferenciável. Uma 1-forma diferencial $\theta$ é dita forma de contato se $\left.\mathrm{d} \theta\right|_{\mathcal{D}_{m !}}$ é não-degenerada, $\forall m \in M$, onde $\mathcal{D}=\operatorname{ker} \theta$, isto é, se $X \in \mathcal{D}_{m}$, existe $Y \in \mathcal{D}_{m}$ tal que $\mathrm{d} \theta(X, Y) \neq 0$.

Observe inicialmente que o fato de $\left.\mathrm{d} \theta\right|_{\mathcal{D}}$ ser forma bilinear anti-simétrica implica que $\operatorname{dim} \mathcal{D}$ seja par e que portanto $\operatorname{dim} M$ seja ímpar. De fato, se tomarmos um produto interno em $\mathcal{D}_{m}$, a matriz $A$, que representa $\left.\mathrm{d} \theta\right|_{\mathcal{D}_{m}}$ em relação a uma base qualquer, é anti-simétrica e então, $\operatorname{det} A=\operatorname{det}\left(-A^{t}\right)=(-1)^{n} \operatorname{det} A$. Como $\operatorname{det} A \neq 0,\left.\operatorname{pois} \mathrm{d} \theta\right|_{\mathcal{D}_{m}}$ é não-degenerada, segue que $n$ é necessariamente par. 
Note também que o fato de $\left.\mathrm{d} \theta\right|_{\mathcal{D}}$ ser não-degenerada equivale a que $\left(\left.\mathrm{d} \theta\right|_{\mathcal{D}}\right)^{n}$ ser nãodegenerada, onde $\operatorname{dim} \mathcal{D}=2 n$. De fato, não é difícil verificar que, se $\left(\theta^{i}\right), i=1, \ldots, 2 n$, é base de $\mathcal{D}_{m}^{*}$ e d $\left.\theta\right|_{\mathcal{D}_{m}}=\sum a_{i j} \theta^{i} \wedge \theta^{j}$, o fato $\left.\operatorname{de} \mathrm{d} \theta\right|_{\mathcal{D}_{m}}$ ser não-degenerada equivale a dizer que $\operatorname{det} A \neq 0$, onde $A$ é a matriz antisimétrica dada por $A=\left(a_{i j}\right)$. Mas, utilizando algumas propriedades de determinantes, obtemos que

$$
\left(\left.\mathrm{d} \theta\right|_{\mathcal{D}}\right)^{n}=2^{n} n !(\operatorname{det} A)^{\frac{1}{2}} \theta^{1} \wedge \cdots \wedge \theta^{2 n}
$$

Portanto, $\left.\mathrm{d} \theta\right|_{\mathcal{D}}$ é não-degenerada se e somente se $\left(\left.\mathrm{d} \theta\right|_{\mathcal{D}}\right)^{n}$ não-degenerada.

Definição 1.2 Seja $M$ uma variedade diferenciável e $\mathcal{D}$ uma distribuição, igualmente diferenciável, de codimensão 1. Fixado $m \in M$, considere a aplicação bilinear e antisimétrica, induzida pelo colchete de campos $[,]_{\mathcal{D}_{m}}: \mathcal{D}_{m} \times \mathcal{D}_{m} \rightarrow T_{m} M / \mathcal{D}_{m}$, dada por

$$
[X, Y]_{\mathcal{D}_{m}}=[\widetilde{X}, \tilde{Y}]_{(m)} \bmod \mathcal{D}_{m}
$$

onde $\widetilde{X}$ e $\tilde{Y}$ são campos definidos em uma vizinhança de $m$, contidos em $\mathcal{D}$, tais que $\widetilde{X}(m)=X$ e $\tilde{Y}(m)=Y$.

Um simples cálculo nos mostra que a aplicação acima está bem definida, isto é, que o resultado independe das extensões $\widetilde{X}$ e $\tilde{Y}$ tomadas. Uma tal distribuição então é dita uma distribuição de contato $s e[,]_{\mathcal{D}_{m}}$ é não-degenerada para todo $m \in M$.

Note que se $\theta$ é uma forma de contato, $\mathcal{D}=\operatorname{ker} \theta$ será uma distribuição de contato. De fato, se $X, Y \in \mathcal{D}_{m}$ e $v \in T_{m} M$ é tal que $\theta(v)=1$,

$$
\begin{aligned}
{[X, Y]_{\mathcal{D}_{m}} } & =[\widetilde{X}, \tilde{Y}]_{m} \bmod \mathcal{D}_{m} \\
& =\theta\left([\widetilde{X}, \tilde{Y}]_{m}\right) v \bmod \mathcal{D}_{m} \\
& =\left[\widetilde{X}(\theta(\tilde{Y}))_{(m)}-\tilde{Y}(\theta(\widetilde{X}))_{(m)}-\mathrm{d} \theta(\widetilde{X}, \tilde{Y})_{(m)}\right] v \bmod \mathcal{D}_{m} \\
& =-\mathrm{d} \theta(X, Y) v \bmod \mathcal{D}_{m},
\end{aligned}
$$

desde que $\theta(\widetilde{X}) \equiv \theta(\widetilde{Y})=0$. Portanto, o fato $\operatorname{de} \mathrm{d} \theta$ ser não-degenerada implica que $[,]_{\mathcal{D}_{m}}$ é não-degenerada. 
Reciprocamente, se $\mathcal{D}$ for uma distribuição de contato, transversalmente orientável (isto é, se admitir um recobrimento por cartas $\left\{\left(U_{\alpha}, \varphi_{\alpha}, \theta^{\alpha}\right)\right\}_{\alpha \in I}$, onde $\operatorname{ker} \theta_{m}^{\alpha}=\mathcal{D}_{m}$, $\forall m \in U_{\alpha}$, tal que $\theta^{i}=f_{i j} \theta^{j} \operatorname{com} f_{i j}>0$ em $U_{i} \cap U_{j}, \forall i, j \in I$ tais que $U_{i} \cap U_{j} \neq \emptyset$ ), então podemos construir, via partição da unidade, uma 1 -forma $\theta$ tal que $\operatorname{ker} \theta=\mathcal{D}$. Pelo visto acima, o fato de $\mathcal{D}$ ser de contato implicará que $\theta$ seja de contato.

Note também que a exigência de orientabilidade transversal de $\mathcal{D}$ não chega a constituir grande restrição, pois, se $\mathcal{D}$ não for transversalmente orientável, podemos obter um recobrimento duplo $\left(M^{\prime}, \pi\right)$ para $M$, onde $\mathcal{D}^{\prime}$, dada por $\mathcal{D}_{p}^{\prime}=\left(\mathrm{d} \pi_{p}\right)^{-1}\left(\mathcal{D}_{\pi(p)}\right)$, será uma distribuição de contato, transversalmente orientável. A construção é análoga à feita para orientação de variedades ou de folheações (ver [3] p.34 e [1] p.35, respectivamente). A partir desse ponto, assumiremos sempre nossa distribuição transversalmente orientável.

Talvez seja interessante ressaltar a certa oposição entre as definições de distribuição de contato e distribuição involutiva (de codimensão 1). Lembremos que $\mathcal{D}$ (de codimensão 1) ser involutiva significa que a operação induzida pelo colchete $[,]_{\mathcal{D}_{m}}$ é identicamente nula para todo $m \in M$. Se denotarmos por $L_{m}$ o conjunto das aplicações lineares de $\mathcal{D}_{m}$ em $T_{m} M / \mathcal{D}_{m}$ e por $\varphi_{m}$ a aplicação $\varphi_{m}: \mathcal{D}_{m} \rightarrow L_{m}$, dada por $\varphi_{m}(X)(Y):=[X, Y]_{\mathcal{D}_{m}}$, então $\mathcal{D}$ ser involutiva significa $\varphi_{m}=0, \forall m \in M$, e $\mathcal{D}$ ser de contato, que $\varphi_{m}(X) \not \equiv 0$, $\forall m \in M, \forall X \in \mathcal{D}_{m}-\{0\}$.

Definição 1.3 Uma variedade sub-Riemanniana de contato é uma variedade diferenciável $M$, junto a uma distribuição de contato $\mathcal{D}$ em $M$ e uma métrica diferenciável $\langle$, definida em $\mathcal{D}$.

Recordemos que uma métrica $\langle$,$\rangle em \mathcal{D}$ é uma correspondência que, a cada ponto $m \in M$ associa um produto interno $\langle,\rangle_{m}$ em $\mathcal{D}_{m}$. A diferenciabilidade de $\langle$,$\rangle é no sentido$ de que, dados dois campos em $\mathcal{D}, X$ e $Y$, diferenciáveis, a aplicação $\varphi(m)=\left\langle X_{m}, Y_{m}\right\rangle_{m}$, definida na interseção dos domínios de $X$ e $Y$, é diferenciável.

Observe que a rigor precisaríamos denotar uma variedade sub-Riemanniana por uma terna $(M, \mathcal{D},\langle\rangle$,$) , mas, por uma questão de simplificação do texto, denotaremos simples-$ 
mente por $M$ uma tal variedade, sempre que tal notação não acarrete dúvidas.

Note agora que, dada uma variedade sub-Riemanniana de contato $(M, \mathcal{D},\langle\rangle$,$) , exis-$ tem infinitas formas de contato $\theta$, tais que $\operatorname{ker} \theta=\mathcal{D}$. De fato, se $\theta$ é forma de contato e $f: M \rightarrow \mathbb{R}$ uma função diferenciável que não se anula em nenhum ponto de $M$, então $\theta^{\prime}=f \theta$ é também forma de contato com $\operatorname{ker} \theta^{\prime}=\mathcal{D}$. O fato de $\mathcal{D}$ possuir uma métrica no entanto nos permite "normalizar" a forma de contato, da seguinte maneira:

Definição 1.4 Dada uma variedade sub-Riemanniana de contato $M$, chamamos de forma de contato associada a $M$ à (única) 1-forma $\theta$ tal que

(i) $\operatorname{ker} \theta=\mathcal{D}$;

(ii) $\left(\left.\mathrm{d} \theta\right|_{\mathcal{D}}\right)^{n}=2^{n} n ! \mathrm{d} V$,

onde $\mathrm{d} V$ denota a forma volume em $\mathcal{D}$.

A partir de então, será indiferente nos referirmos a uma variedade sub-Riemanniana de contato por $(M, \mathcal{D},\langle\rangle$,$) ou (M, \theta,\langle\rangle$,$) .$

Uma outra conseqüência importante da estrutura de contato nos é garantida pela seguinte

Proposição 1.1 Dada uma variedade sub-Riemanniana de contato $(M, \mathcal{D}, g)$ com forma de contato associada $\theta$, existe um único campo $\xi$ em $M$ tal que

(i) $\theta(\xi)=1$;

(ii) $\iota_{\xi} \mathrm{d} \theta=0$

Prova: Existência local - dado $x \in M$, tome, em um aberto suficientemente pequeno $U \ni x$, um campo $X$ tal que $\theta(X)=1$ (isso é sempre possível de ser feito). Considere 
então a 1-forma em $U$, dada por

$$
\tau(v)=\mathrm{d} \theta(X, v) .
$$

Ora, então em cada ponto $m \in U$, a restrição de $\tau_{m}$ a $\mathcal{D}_{m}$ é elemento de $\mathcal{D}_{m}^{*}$. Mas, considerando agora a aplicação $\varphi_{m}: \mathcal{D}_{m} \rightarrow \mathcal{D}_{m}^{*}$, dada por

$$
\varphi_{m}(v)(t)=\mathrm{d} \theta_{m}(v, t), \quad v, t \in \mathcal{D}_{m},
$$

observe que o fato de $\left.\mathrm{d} \theta\right|_{\mathcal{D}}$ ser não-degenerada implica que $\varphi_{m}$ é isomorfismo. Logo, em cada $m \in U$, existe um único $\bar{v}_{m} \in \mathcal{D}_{m}$ tal que $\varphi\left(\bar{v}_{m}\right)=\tau_{m}$, ou seja, que

$$
\begin{aligned}
& \mathrm{d} \theta_{m}\left(\bar{v}_{m}, t\right)=\mathrm{d} \theta_{m}\left(X_{m}, t\right), \forall t \in \mathcal{D}_{m} \\
& \text { ou } \quad \mathrm{d} \theta_{m}\left(X_{m}-\bar{v}_{m}, t\right)=0 .
\end{aligned}
$$

Note que a diferenciabilidade do campo $\bar{v}$ é decorrência da diferenciabilidade das formas $\theta$ e $\mathrm{d} \theta$. Basta então tomarmos $\xi=X-\bar{v}$, para termos

(i) $\theta(\xi)=\theta(X)-\theta(\bar{v})=1-0=1$;

(ii) $\iota_{\xi}(\mathrm{d} \theta)=0$.

Unicidade: Suponha que existam dois campos em um aberto $U, \xi$ e $\xi^{\prime}$, que satisfaçam (i) e (ii). Então teremos que $\xi-\xi^{\prime} \in \mathcal{D}$. De fato, teremos que $\theta\left(\xi-\xi^{\prime}\right)=\theta(\xi)-\theta\left(\xi^{\prime}\right)=$ $1-1=0$. Também teremos que $\mathrm{d} \theta\left(\xi-\xi^{\prime}, t\right)=0, \forall t \in \mathcal{D}_{m}$. Então, como $\left.\mathrm{d} \theta\right|_{\mathcal{D}}$ é não-degenerada, teremos necessariamente que $\xi-\xi^{\prime}=0$, ou seja, $\xi=\xi^{\prime}$.

Existência do campo global: Fica garantida pela existência e unicidade local.

\subsection{Exemplos}

(1) Espaço de Heisenberg $H^{3}$ 
Considere em $\mathbb{R}^{3}$ a distribuição $\mathcal{D}$ (ver figura adiante) gerada pelos campos

$$
\begin{aligned}
& e_{1}=\frac{\partial}{\partial x}-y \frac{\partial}{\partial z} \\
& e_{2}=\frac{\partial}{\partial y}+x \frac{\partial}{\partial z}
\end{aligned}
$$

munida da métrica que torna $e_{1}, e_{2}$ ortonormais.

Observe inicialmente que $\mathcal{D}$ é de contato, de fato,

$$
\left[e_{1}, e_{2}\right]=2 \frac{\partial}{\partial z} \notin \mathcal{D} \text {. }
$$

Note também que as restrições das 1 -formas $\{\mathrm{d} x, \mathrm{~d} y\}$ a $\mathcal{D}$ formam a base dual a $\left\{e_{1}, e_{2}\right\}$ e portanto a métrica $g$ pode ser dada por

$$
g=\mathrm{d} x^{2}+\mathrm{d} y^{2}
$$

e a forma volume (ou elemento de área, como queira) por

$$
\mathrm{d} V=\mathrm{d} x \wedge \mathrm{d} y .
$$

Para obtermos então a forma de contato $\theta$ associada, basta então que exijamos que

$$
\theta\left(e_{1}\right)=0, \quad \theta\left(e_{2}\right)=0,\left.\quad \mathrm{~d} \theta\right|_{\mathcal{D}}=2 \mathrm{~d} V .
$$

Se tomarmos então $\theta=f \mathrm{~d} x+g \mathrm{~d} y+h \mathrm{~d} z$, as duas primeiras condições nos levarão rapidamente a

$$
\left\{\begin{array}{l}
f=y h \\
g=-x h
\end{array},\right.
$$

de onde concluímos, necessariamente, que

$$
\theta=h(y \mathrm{~d} x-x \mathrm{~d} y+\mathrm{d} z)
$$

e daí que

$$
\mathrm{d} \theta=\mathrm{d} h \wedge(y \mathrm{~d} x-x \mathrm{~d} y+\mathrm{d} z)+h(-2 \mathrm{~d} x \wedge \mathrm{d} y)
$$

e como $y \mathrm{~d} x-x \mathrm{~d} y+\mathrm{d} z$ se anula em $\mathcal{D}$ (pois é múltiplo de $\theta$ ), temos que

$$
\left.\mathrm{d} \theta\right|_{\mathcal{D}}=-2 h \mathrm{~d} x \wedge \mathrm{d} y
$$




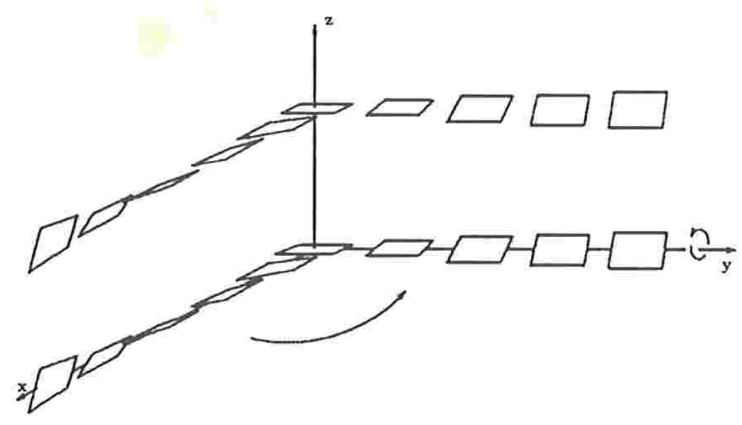

ESPAÇO DE HEISENBERG

$\mathrm{e}$, da condição $\left.\mathrm{d} \theta\right|_{\mathcal{D}}=2 \mathrm{~d} V=2 \mathrm{~d} x \wedge \mathrm{d} y$, temos que $h=-1 \mathrm{e}$

$$
\theta=-y \mathrm{~d} x+x \mathrm{~d} y-\mathrm{d} z
$$

Finalmente, para obtermos o campo $\xi=a \frac{\partial}{\partial x}+b \frac{\partial}{\partial y}+c \frac{\partial}{\partial z}$, $\operatorname{como} \theta(\xi)=1$, temos que

$$
-a y+b x-c=1
$$

e do fato de que $\mathrm{d} \theta\left(\xi, e_{1}\right)=\mathrm{d} \theta\left(\xi, e_{2}\right)=0$, temos

$$
\begin{aligned}
& \mathrm{d} \theta\left(\xi, e_{1}\right)=2 \mathrm{~d} x \wedge \mathrm{d} y\left(\xi, e_{1}\right)=-2 b=0, \text { portanto, } b=0 \\
& \mathrm{~d} \theta\left(\xi, e_{2}\right)=2 \mathrm{~d} x \wedge \mathrm{d} y\left(\xi, e_{2}\right)=2 a=0, \text { portanto, } a=0 .
\end{aligned}
$$

Logo,

$$
\xi=-\frac{\partial}{\partial z}
$$

(2) Esfera $S^{3}$

Considere $\mathbb{C}^{2} \cong \mathbb{R}^{4}$ com o produto hermitiano assim definido:

$$
\left\langle\left(z_{1}, z_{2}\right),\left(w_{1}, w_{2}\right)\right\rangle=z_{1} \bar{w}_{1}+z_{2} \bar{w}_{2}
$$

onde $z_{i}, w_{i} \in \mathbb{C}$. Seja, então,

$$
S^{3}=\left\{\left(z_{1}, z_{2}\right) \in \mathbb{C}^{2}:\left\langle\left(z_{1}, z_{2}\right),\left(z_{1}, z_{2}\right)\right\rangle=1\right\} .
$$


Note que, se fizermos $z_{1}=x+i y$ e $z_{2}=w+i t$, a condição que define $S^{3}$ acima se reduz exatamente a $x^{2}+y^{2}+w^{2}+t^{2}=1$.

Fazendo $z=\left(z_{1}, z_{2}\right) \in \mathbb{C}^{2}$ e $\bar{z}=\left(\bar{z}_{1}, \bar{z}_{2}\right)$, a título de simplificar a notação, teremos

$$
S^{3}=\left\{z \in \mathbb{C}^{2}:\|z\|^{2}=1\right\} .
$$

Precisamos identificar agora o espaço tangente a $S^{3}$ em um ponto $z$ : seja então $\gamma:(-\varepsilon, \varepsilon) \rightarrow S^{3}$ tal que $\gamma(0)=z$, do fato que $\|\gamma(t)\|^{2}=1$, temos que

$$
\begin{aligned}
& \langle\gamma(0), \dot{\gamma}(0)\rangle+\langle\dot{\gamma}(0), \gamma(0)\rangle=0 \\
& \langle\gamma(0), \dot{\gamma}(0)\rangle+\overline{\langle\gamma(0), \dot{\gamma}(0)\rangle}=0 \\
& \operatorname{Re}(\langle\gamma(0), \dot{\gamma}(0)\rangle)=0 .
\end{aligned}
$$

Daqui concluímos então que se $v \in T_{z} S^{3}$, necessariamente, $(z, v):=\operatorname{Re}(\langle z, v\rangle)=0$. A recíproca é também verdadeira: dado um vetor $v$ tal que $(z, v)=0, \exists \gamma:(-\varepsilon, \varepsilon) \rightarrow$ $S^{3}$ tal que $\gamma(0)=z$ e $\dot{\gamma}(0)=v$. De fato, considere a curva $\alpha(t)=z+t v$ e tome então

$$
\begin{aligned}
\gamma(t) & =\frac{\alpha(t)}{\|\alpha(t)\|} \\
\dot{\gamma}(t) & =\frac{1}{\|\alpha(t)\|^{2}}\left\{\|\alpha(t)\| \dot{\alpha}(t)+\frac{\mathrm{d}}{\mathrm{d} t}[\|\alpha(t)\|] \alpha(t)\right\}
\end{aligned}
$$

e como $\|\alpha(0)\|=\|z\|=1, \dot{\alpha}(0)=v$ e $\frac{\mathrm{d}}{\mathrm{d} t}[\|\alpha(t)\|]_{t=0}=0$, pois

$$
\frac{\mathrm{d}}{\mathrm{d} t}\left[\|\alpha(t)\|^{2}\right]=2 \operatorname{Re}(\langle\alpha(t), \dot{\alpha}(t)\rangle)=2(\alpha(t), \dot{\alpha}(t))
$$

e em $t=0$

$$
\frac{\mathrm{d}}{\mathrm{d} t}\left[\|\alpha(t)\|^{2}\right]_{t=0}=2(\alpha(0), \dot{\alpha}(0))=2(z, v)=0,
$$

temos que $\dot{\gamma}(0)=v$. Portanto, concluímos que

$$
T_{z} S^{3}=\left\{v \in \mathbb{C}^{2}:(z, v)=0\right\} .
$$

Considere agora a seguinte distribuição $\mathcal{D}$ em $T S^{3}$ :

$$
\mathcal{D}_{z}=\left\{v \in \mathbb{C}^{2}:\langle z, v\rangle=0\right\}
$$


com a métrica induzida da métrica euclidiana do $\mathbb{C}^{2}$ :

$$
g=\langle\mathrm{d} z, \overline{\mathrm{d} z}\rangle=\mathrm{d} z_{1} \overline{\mathrm{d} z_{1}}+\mathrm{d} z_{2} \overline{\mathrm{d} z_{2}}=\mathrm{d} x_{1}^{2}+\mathrm{d} x_{2}^{2}+\mathrm{d} x_{3}^{2}+\mathrm{d} x_{4}^{2} .
$$

Note que, evidentemente, $\mathcal{D}_{z_{0}} \subset T_{z_{0}} S^{3}$. Além disso, observe que, se $z=\left(z_{1}, z_{2}\right) \mathrm{e}$ tomarmos $z^{\prime}=\left(-\bar{z}_{2}, \bar{z}_{1}\right),\left\{z, z^{\prime}\right\}$ é base unitária de $\mathbb{C}^{2}$ e, além disso, é fácil verificar que

$$
\mathcal{D}_{z}=\left\{\lambda z^{\prime}: \lambda \in \mathbb{C}\right\}
$$

Se escrevermos então $z$ em coordenadas canônicas reais, i.e.,

$$
z=\left(z_{1}, z_{2}\right)=\left(x_{1}+i x_{2}, x_{3}+i x_{4}\right)=\left(x_{1}, x_{2}, x_{3}, x_{4}\right)
$$

observe que, se

$$
\begin{aligned}
z & =\left(x_{1}, x_{2}, x_{3}, x_{4}\right) \\
e_{1}:=z^{\prime} & =\left(-x_{3}, x_{4}, x_{1},-x_{2}\right) \\
e_{2}:=i z^{\prime} & =\left(-x_{4},-x_{3}, x_{2}, x_{1}\right) \\
e_{3}:=i z & =\left(-x_{2}, x_{1},-x_{4}, x_{3}\right)
\end{aligned}
$$

então $\left\{z, e_{1}, e_{2}, e_{3}\right\}$ é base ortonormal de $\mathbb{R}^{4}$, além disso, $\left\{e_{1}, e_{2}\right\}$ é base ortonormal de $\mathcal{D}_{z}$ e como

$$
\left\langle z, e_{3}\right\rangle=\langle z, i z\rangle=\tau\langle z, z\rangle=-i \neq 0
$$

segue que $e_{3} \notin \mathcal{D}_{z}$, mas como $\left(z, e_{3}\right)=\operatorname{Re}\left(\left\langle z, e_{3}\right\rangle\right)=0$, temos que $e_{3} \in T_{3} S^{3}$.

Tomando então a base dual de $\left\{z, e_{1}, e_{2}, e_{3}\right\}$, obtemos, ordenadamente,

$$
\begin{aligned}
\Phi & =x_{1} \mathrm{~d} x_{1}+x_{2} \mathrm{~d} x_{2}+x_{3} \mathrm{~d} x_{3}+x_{4} \mathrm{~d} x_{4}, \\
\theta^{1} & =-x_{3} \mathrm{~d} x_{1}+x_{4} \mathrm{~d} x_{2}+x_{1} \mathrm{~d} x_{3}-x_{2} \mathrm{~d} x_{4}, \\
\theta^{2} & =-x_{4} \mathrm{~d} x_{1}-x_{3} \mathrm{~d} x_{2}+x_{2} \mathrm{~d} x_{3}+x_{1} \mathrm{~d} x_{4}, \\
\theta^{3} & =-x_{2} \mathrm{~d} x_{1}+x_{1} \mathrm{~d} x_{2}-x_{4} \mathrm{~d} x_{3}+x_{3} \mathrm{~d} x_{4} .
\end{aligned}
$$

Note então que as restrições de $\theta^{1}$ e $\theta^{2}$ a $\mathcal{D}$ nos dão a base dual de $\left\{e_{1}, e_{2}\right\}$ e a restrição de $\theta^{3}$ a $S^{3}$ é a forma de contato associada a $\mathcal{D}$, de fato,

(i) $\theta^{3}(\mathcal{D})=0 \mathrm{e}$ 
(ii) $\mathrm{d} \theta\left(e_{1}, e_{2}\right)=2\left(\mathrm{~d} x_{1} \wedge \mathrm{d} x_{2}+\mathrm{d} x_{3} \wedge \mathrm{d} x_{4}\right)\left(e_{1}, e_{2}\right)=2\left(x_{3}^{2}+x_{4}^{2}+x_{1}^{2}+x_{2}^{2}\right)=2=$ $2 \mathrm{~d} V\left(e_{1}, e_{2}\right)$

e, além disso, $e_{3}=\xi$ é o campo fundamental, pois

(i) $\theta^{3}\left(e_{3}\right)=1$,

(ii) $\mathrm{d} \theta^{3}\left(e_{3}, e_{i}\right)=0, \quad i=1,2$.

\section{Observações}

Em $S^{3}$ considere a seguinte relação:

$$
z \sim w \Leftrightarrow \exists \lambda \in S^{1} \text { tal que } z=\lambda w, z, w \in S^{3} \subset \mathbb{C}^{2},
$$

onde o produto de $\lambda$ por $w=\left(w_{1}, w_{2}\right)$ é dado por $\lambda w=\left(\lambda w_{1}, \lambda w_{2}\right)$. É trivial notar que tal relação é de equivalência e que $z \in S^{3} \Rightarrow \lambda z \in S^{3}, \forall \lambda \in S^{1}$.

Definimos então $\mathbb{C} P^{1}=\left\{[z] ; z \in S^{3}\right\}=$ conjunto das classes de equivalência de $S^{3}$ em relação a $\sim$.

Considere agora a projeção $\pi: S^{3} \rightarrow \mathbb{C} P^{1}$. Queremos observar aqui somente o fato de que $\left.\mathrm{d} \pi_{z}\right|_{\mathcal{D}_{z}}: \mathcal{D}_{z} \rightarrow T_{[z]} \mathbb{C} P^{1}$ é um isomorfismo linear. De fato,

$$
\mathrm{d} \pi_{z}: T_{z} S^{3} \rightarrow T_{[z]} \mathbb{C} P^{1}
$$

é evidentemente sobrejetora e além disso, note que

$$
e_{3}=i z=\left.\frac{\mathrm{d}}{\mathrm{d} t}\left(e^{i t} z\right)\right|_{t=0}
$$

e portanto

$$
\begin{aligned}
\mathrm{d} \pi_{z}\left(e_{3}\right) & =\left.\mathrm{d} \pi_{z}\left(\frac{\mathrm{d}}{\mathrm{d} t} e^{i t} z\right)\right|_{t=0}=\frac{\mathrm{d}}{\mathrm{d} t}\left[\pi\left(e^{i t} z\right)\right]_{t=0} \\
& =\left.\frac{\mathrm{d}}{\mathrm{d} t}([z])\right|_{t=0}=0
\end{aligned}
$$

Portanto, como $T_{z} S^{3}=\left[e_{3 z}\right] \oplus \mathcal{D}_{z}$, temos que $\left.\mathrm{d} \pi_{z}\right|_{\mathcal{D}_{z}}: \mathcal{D}_{z} \rightarrow T_{[z]} \mathbb{C} P^{1}$ é isomorfismo linear.

É fácil verificar que

$$
\pi^{-1}([z])=\left\{e^{i \theta} z \mid \theta \in \mathbb{R}\right\}=S^{1}
$$


e portanto as fibras são grupos abelianos e o fibrado $\left(S^{3}, \pi, C P^{1}\right)$ de $\mathbb{C} P^{1}$ é fibrado principal, chamado fibrado de Hopf.

Queremos salientar aqui o fato de que a fibração em questão pode ser vista naturalmente como uma fibração sobre $S^{2}$ ao invés de $\mathbb{C} P^{1}$. Isso se dá pelo fato de $\mathbb{C} P^{1} \mathrm{e}$ $S^{2}$ serem difeomorfos. Tal difeomorfismo não é difícil de construir, bastando tomar o sistema de coordenadas não-homogêneo de $\mathbb{C} P^{1}$,

$$
\begin{aligned}
C: \mathbb{C} P^{1} & \rightarrow \mathbb{C} \cup\{\infty\} \\
C\left(\left[z_{1}, z_{2}\right]\right) & = \begin{cases}\frac{z_{1}}{z_{2}} & , \text { se } z_{2} \neq 0 \\
\infty & , \text { se } z_{2}=0\end{cases}
\end{aligned}
$$

e compor com a inversa da projeção estereográfica do $S^{2}$.

Note também que a aplicação $R_{\theta}: S^{3} \rightarrow S^{3}$ dada por $R_{\theta}(z)=e^{i \theta} z$ é isometria, pois $\mathrm{d} R_{\theta}(v)=e^{i \theta} v \mathrm{e}$

$$
\left.\left\langle\mathrm{d} R_{\theta}(v), \mathrm{d} R_{\theta}(w)\right\rangle\right)=\left\langle e^{i \theta} v, e^{i \theta} w\right\rangle=e^{i \theta} \overline{e^{i \theta}}\langle v, w\rangle=\langle v, w\rangle .
$$

Podemos então construir uma métrica $\langle$,$\rangle para \mathbb{C} P^{1}$, "projetando" a métrica de $\mathcal{D}_{z}:$ se $w, t \in T_{[z]} \mathbb{C} P^{1}$ e $u, v \in \mathcal{D}_{z}$ tal que $\mathrm{d} \pi_{z}(u)=w$ e $\mathrm{d} \pi_{z}(v)=t$, definimos

$$
\ll w, t \gg=\langle u, v\rangle \text {. }
$$

Essa métrica é chamada de métrica de Fubini-Study.

(3) Quádrica $Q^{3}$

Considere agora o seguinte produto em $\mathbb{C}^{2}$

$$
\left\langle\left(z_{1}, z_{2}\right),\left(w_{1}, w_{2}\right)\right\rangle=z_{1} \bar{w}_{1}-z_{2} \bar{w}_{2} .
$$

Definimos $Q^{3}$ como sendo

$$
Q^{3}=\left\{z \in \mathbb{C}^{2}:\langle z, z\rangle=1\right\}
$$

ou, em coordenadas reais,

$$
Q^{3}=\left\{\left(x_{1}, x_{2}, x_{3}, x_{4}\right) \in \mathbb{R}^{4}: x_{1}^{2}+x_{2}^{2}-x_{3}^{2}-x_{4}^{2}=1\right\}
$$


De forma análoga ao feito para $S^{3}$, mostramos que

$$
T_{z} Q^{3}=\left\{v \in \mathbb{C}^{2}:(z, v)=0\right\}
$$

onde $(z, v)=\operatorname{Re}(\langle z, v\rangle)$, definimos a distribuição

$$
\mathcal{D}_{z}=\left\{v \in \mathbb{C}^{2} \mid\langle z, v\rangle=0\right\}
$$

e a métrica em $\mathcal{D}_{z}$ por

$$
\begin{aligned}
g:=-\langle\mathrm{d} z, \mathrm{~d} z\rangle & =\mathrm{d} z_{2} \overline{\mathrm{d}} z_{2}-\mathrm{d} z_{1} \overline{\mathrm{d}} z_{1} \\
& =\mathrm{d} x_{3}^{2}+\mathrm{d} x_{4}^{2}-\mathrm{d} x_{1}^{2}-\mathrm{d} x_{2}^{2}
\end{aligned}
$$

É claro que $\mathcal{D}_{z} \subset T_{z} Q^{3}, \forall z \in H^{3}$. Observe agora que $z=\left(z_{1}, z_{2}\right)$ e $z^{\prime}=\left(\bar{z}_{2}, \bar{z}_{1}\right)$ formam uma base unitária de $\mathbb{C}^{2}$. Considere então os vetores

$$
\begin{aligned}
z & =\left(x_{1}, x_{2}, x_{3}, x_{4}\right) \\
e_{3}=-i z & =\left(x_{2},-x_{1}, x_{4},-x_{3}\right) \\
e_{1}=z^{\prime} & =\left(x_{3},-x_{4}, x_{1},-x_{2}\right) \\
e_{2}=i z^{\prime} & =\left(x_{4}, x_{3}, x_{2}, x_{1}\right)
\end{aligned}
$$

Repare que $\left\{z, e_{1}, e_{2}, e_{3}\right\}$ é base de $\mathbb{R}^{4}$, que $\left\{e_{1}, e_{2}\right\}$ é base ortonormal de $\mathcal{D}$, que a métrica $g$ está bem definida (pois $g\left(e_{1}\right)=g\left(e_{2}\right)=1$ ) e que $\left\{e_{1}, e_{2}, e_{3}\right\}$ é base de $T Q^{3}$. Dualizando então obtemos

$$
\begin{aligned}
\Phi & =x_{1} \mathrm{~d} x_{1}+x_{2} \mathrm{~d} x_{2}-x_{3} \mathrm{~d} x_{3}-x_{4} \mathrm{~d} x_{4}, \\
\theta^{1} & =-x_{3} \mathrm{~d} x_{1}+x_{4} \mathrm{~d} x_{2}+x_{1} \mathrm{~d} x_{3}-x_{2} \mathrm{~d} x_{4}, \\
\theta^{2} & =-x_{4} \mathrm{~d} x_{1}-x_{3} \mathrm{~d} x_{2}+x_{2} \mathrm{~d} x_{3}+x_{1} \mathrm{~d} x_{4}, \\
\theta^{3} & =x_{2} \mathrm{~d} x_{1}-x_{1} \mathrm{~d} x_{2}-x_{4} \mathrm{~d} x_{3}+x_{3} \mathrm{~d} x_{4} .
\end{aligned}
$$

Da mesma forma as restrições de $\theta^{1}$ e $\theta^{2}$ a $\mathcal{D}$ nos dão a base dual de $\left\{e_{1}, e_{2}\right\}$ e $\theta^{3}$ é a forma de contato associada a $\mathcal{D}$, pois

(i) $\theta^{3}(\mathcal{D})=0$

(ii) $\mathrm{d} \theta^{3}\left(e_{1}, e_{2}\right)=2\left(-\mathrm{d} x_{1} \wedge \mathrm{d} x_{2}+\mathrm{d} x_{3} \wedge \mathrm{d} x_{4}\right)\left(e_{1}, e_{2}\right)=2=2 \mathrm{~d} V\left(e_{1}, e_{2}\right)$. 
e finalmente é claro que $e_{3}$ é o campo fundamental $\xi$, pois

(i) $\theta^{3}\left(e_{3}\right)=1$,

(ii) $\mathrm{d} \theta^{3}\left(e_{3}, e_{i}\right)=0, i=1,2$.

Observe que, utilizando a mesma relação $\sim$ definida no exemplo 1.2 e procedendo de forma idêntica, podemos mostrar que $\mathrm{d} \pi_{z}: \mathcal{D}_{z} \rightarrow T_{[z]} Q^{3} / \sim$ é isomorfismo linear. Além disso, vale ressaltar aqui o fato de que $Q^{3} / \sim$ é difeomorfo a $D^{2}=\{z \in \mathbb{C}$ : $\|z\|<1\}$ e o difeomorfismo é dado de forma parecida ao caso do exemplo 1.2 , utilizando as coordenadas não homogêneas de $Q^{3} / \sim$ :

$$
\begin{aligned}
C: Q^{3} / \sim & \rightarrow D^{2} \\
{\left[\left(z_{1}, z_{2}\right)\right] } & \mapsto \frac{z_{2}}{z_{1}}
\end{aligned}
$$

(4) Grupo de Lie $G$

Seja $G$ um grupo de Lie de dimensão 3, com álgebra de Lie $\varrho \simeq T_{e} G$ e sejam $X, Y \in \varrho$ tais que $[X, Y] \notin \mathcal{D}$, onde $\mathcal{D}$ denota a distribuição gerada por $\{X, Y\}$. Considere então o produto interno $<,>$ em $\mathcal{D}_{e}$ que torna $X_{e}$ e $Y_{e}$ ortonormais e "espalhemos" esse produto de modo a obtermos em $\mathcal{D}$ uma métrica invariante (à esquerda) pela ação do grupo: se $v_{1}, v_{2} \in \mathcal{D}_{g}$, basta-nos definir

$$
<v_{1}, v_{2}>=<\mathrm{d} L_{g^{-1}}\left(v_{1}\right), \mathrm{d} L_{g^{-1}}\left(v_{2}\right)>
$$

É fácil ver que $(G, \mathcal{D},<,>)$ é variedade sub-Riemanniana de contato e que, se $\left(\theta^{1}, \theta^{2}, \theta^{3}\right)$ for a base dual a $(X, Y,[X, Y])$, então a forma de contato associada será

$$
\theta=-2 \theta^{3}
$$

e que o campo fundamental será

$$
\xi=-\frac{1}{2}\left\{[X, Y]-\theta^{3}([[X, Y], Y]) \cdot X+\theta^{3}([[X, Y], X]) \cdot Y\right\}
$$




\section{Capítulo 2}

\section{Conexão sub-Riemanniana}

A partir desse capítulo, nosso estudo ficará restrito às variedades sub-Riemannianas de contato de dimensão 3.

Neste capítulo, define-se, na primeira seção, o fibrado de círculo $B(M)$ de uma variedade $M$ e é introduzida a noção de conexão nesse fibrado. Na seção 2.2 , é definido o transporte paralelo de um vetor ao longo de uma curva, necessário para se ter uma interpretação geométrica da derivada covariante, definida no Capítulo 3.

Na seção 2.3, são apresentadas as equações de estrutura, o teorema de existência e unicidade da conexão sub-Riemanniana e são definidas a curvatura e a torsão (subRiemannianas) de $M$, enquanto que, na seção 2.4 , são apresentadas as identidades de Bianchi relativas à conexão sub-Riemanniana.

Por fim, na seção 2.5, são determinadas as formas de conexão sub-Riemanniana, as respectivas equações de estrutura, curvaturas e torsão dos exemplos definidos na seção $1.2, H^{3}, S^{3}$ e $Q^{3}$. 


\subsection{Fibrado de círculo, conexão, forma de conexão}

Considere $(M, \mathcal{D},\langle\rangle$,$) uma variedade sub-Riemanniana de contato de dimensão 3$ com forma de contato associada $\theta$. Definimos

$$
B(M)=\{v \in \mathcal{D}:\langle v, v\rangle=1\}
$$

É simples ver que $B(M)$ é variedade diferenciável. Basta observar que 1 é valor regular de $f: \mathcal{D} \rightarrow \mathbb{R}$, onde $f(v)=\langle v, v\rangle$. De fato, se $v \in B(M)$ e $\alpha:(-\varepsilon, \varepsilon) \rightarrow \mathcal{D}$ é a curva dada por $\alpha(t)=(1+t) v$ então

$$
\mathrm{d} f_{v}(\dot{\alpha}(0))=\left.\frac{\mathrm{d}}{\mathrm{d} t} f \circ \alpha\right|_{t=0}=\left.\frac{\mathrm{d}}{\mathrm{d} t}(1+t)^{2}\right|_{t=0}=2 \neq 0
$$

e o Teorema da Função Implícita se aplica (ver [11], p.31). Observe que, ainda do mesmo teorema, segue que $\operatorname{dim} B(M)=4$. (Note que utilizamos o fato de $\mathcal{D}$ ser variedade diferenciável de dimensão 5.)

Supondo então $\mathcal{D}$ transversalmente orientável e $M$ também orientável (caso contrário podemos passar ao recobrimento duplo orientável), é simples notar que podemos fixar uma orientação para $\mathcal{D}$. Basta, por exemplo, que definamos $\left(e_{1}, e_{2}\right)$, base ortonormal de $\mathcal{D}_{m}$, como positiva se, e só se, $\left(e_{1}, e_{2}, \xi\right)$ for base positiva de $T_{m} M$ em relação a uma orientação pré-fixada para $M$. Fixando então uma tal orientação para $\mathcal{D}$, podemos definir agora uma ação de $S^{1}$ sobre $B(M)$ :

$$
\begin{aligned}
S^{1} \times B(M) & \rightarrow B(M) \\
\left(R_{\theta}, v\right) & \mapsto R_{\theta} v
\end{aligned}
$$

onde $R_{\theta}=e^{i \theta} \in S^{1}$ e $R_{\theta} v$ é o vetor de $\mathcal{D}$ obtido a partir da rotação de $v$ de um ângulo $\theta$ no sentido da orientação de $\mathcal{D}$. É evidente que tal aplicação é diferenciável e que $R_{\alpha}\left(R_{\theta} v\right)=\left(R_{\alpha} R_{\theta}\right) v$, que nos mostra ser, de fato, uma ação de $S^{1}$ sobre $B(M)$.

Observe que, se denotarmos por $\pi$ a projeção natural de $B(M)$ sobre $M, \pi^{-1}(m) \cong S^{1}$ e $S^{1}$ age transitivamente em cada fibra $\pi^{-1}(m)$, isto é, dado $v_{1}$ e $v_{2} \in \pi^{-1}(m)$, existe $R_{\theta} \in S^{1}$ tal que $R_{\theta} v_{1}=v_{2}$. 
Definição 2.1 Seja $\mathcal{H}$ uma distribuição diferenciável em $T B(M)$. H será dita uma conexão em $B(M)$ se as duas propriedades abaixo são satisfeitas:

(a) $\mathrm{d} R_{\theta}\left(\mathcal{H}_{p}\right)=\mathcal{H}_{R_{\theta} p}, \quad \forall R_{\theta} \in S^{1}, \forall p \in B(M)$;

(b) $\mathcal{H}$ é horizontal, isto é, para todo $p \in B(M)$,

$$
T_{p} B(M)=\mathcal{H}_{p} \oplus \mathrm{d} \pi_{p}^{-1}(0)
$$

onde $\mathrm{d} \pi_{p}^{-1}(0)=\left\{v \in T_{p} B(M): \mathrm{d} \pi(v)=0\right\}$.

Observe que o fato de $\pi: B(M) \rightarrow M$ ser submersão, garante-nos que $\mathrm{d} \pi_{p}^{-1}(0)$ é subespaço unidimensional de $T_{p} B(M)$, qualquer que seja $p \in B(M)$.

Afirmamos agora que existe um campo diferenciável $V$, definido em $B(M)$, que gera $\mathrm{d} \pi^{-1}(0)$ :

$$
V_{(p)}=\left.\frac{\mathrm{d}}{\mathrm{d} t}\left(R_{t} p\right)\right|_{t=0}
$$

$V(p)$ é evidentemente não nulo e

$$
\mathrm{d} \pi_{p}\left(V_{p}\right)=\mathrm{d} \pi\left[\left.\left(\frac{\mathrm{d}}{\mathrm{d} t} R_{t} p\right)\right|_{t=0}\right]=\left.\frac{\mathrm{d}}{\mathrm{d} t}\left(\pi\left(R_{t} p\right)\right)\right|_{t=0}=\left.\frac{\mathrm{d}}{\mathrm{d} t}(\pi(p))\right|_{t=0}=0
$$

Definição 2.2 Uma forma de conexão em $B(M)$ é uma 1-forma diferenciável $\omega$ tal que

(i) $R_{\theta}^{*} \omega=\omega$;

(ii) $\omega(V)=1$.

Proposição 2.1 Se $\omega$ é uma forma de conexão em $B(M)$, então $\operatorname{ker} \omega$ é conexão em $B(M)$. Por outro lado, se $\mathcal{H}$ é uma conexão em $B(M)$, existe uma única forma de conexão $\omega$ tal que $\mathcal{H}=\operatorname{ker} \omega$. 
Prova: Suponha $\omega$ forma de conexão, então $\mathcal{H}=$ ker $\omega$ é distribuição diferenciável em $B(M)$ de codimensão 1 . Além disso, se $v \in \mathcal{H} \cap \mathrm{d} \pi^{-1}(0)$, então $v=\lambda V$ e $\omega(v)=0$, mas então $\lambda=\lambda \omega(V)=\omega(v)=0$ e portanto $v=0$ e $T B(M)=\mathcal{H} \oplus \mathrm{d} \pi^{-1}(0)$. Note ainda que

$$
\omega_{R_{\theta} p}\left(\mathrm{~d} R_{\theta}\left(\mathcal{H}_{p}\right)\right)=\left(R_{\theta}^{*} \omega\right)_{p}\left(\mathcal{H}_{p}\right)=\omega_{p}\left(\mathcal{H}_{p}\right)=0
$$

Então, como $R_{\theta}$ é o difeomorfismo (basta notar que $R_{\theta} \circ R_{-\theta}=I_{d}$ ), segue que $\mathrm{d} R_{\theta}\left(\mathcal{H}_{p}\right)=$ $\mathcal{H}_{R_{\theta}}$ e portanto $\mathcal{H}_{p}$ satisfaz (a) e (b).

Suponha agora que $\mathcal{H}$ seja uma conexão em $B(M)$. Definamos então $\omega$ da seguinte forma: se $v \in T_{p} B(M)=\mathcal{H}_{p} \oplus \mathrm{d} \pi_{p}^{-1}(0)$, então $v$ se escreve de forma única como

$$
v=h+\lambda V_{p} \text {, onde } h \in \mathcal{H}_{p} \quad \text { e } \quad \lambda \in \mathbb{R} .
$$

Defina então $\omega(v)=\lambda$. É claro que $\omega$ é diferenciável, pois, se $\left(X_{1}, X_{2}, X_{3}\right)$ são campos diferenciáveis que geram $\mathcal{H}$ em um aberto $U$ de $B(M)$ e $\left(\theta^{1}, \theta^{2}, \theta^{3}, \theta^{4}\right)$ é a base dual a $\left(X_{1}, X_{2}, X_{3}, V\right)$, é evidente que $\omega$ coincide com $\theta^{4}$ em $U$ e é portanto diferenciável. É claro então que $\mathcal{H}=\operatorname{ker} \omega$. Note ainda que $\omega(V)=1$ e que

$$
\left(R_{\theta}^{*} \omega\right)_{p}\left(h+\lambda V_{p}\right)=\omega_{R_{\theta} p}\left(\mathrm{~d} R_{\theta}(h)+\lambda \mathrm{d} R_{\theta}\left(V_{p}\right)\right)
$$

Agora, como $\mathrm{d} R_{\theta}\left(\mathcal{H}_{p}\right)=\mathcal{H}_{R_{\theta} p}$, segue que $\mathrm{d} R_{\theta}(h)=h^{\prime} \in \mathcal{H}_{R_{\theta} p}$. Além disso,

$$
\mathrm{d} R_{\theta}\left(V_{p}\right)=\mathrm{d} R_{\theta}\left[\left.\frac{\mathrm{d}}{\mathrm{d} t}\left(R_{t} p\right)\right|_{t=0}\right]=\frac{\mathrm{d}}{\mathrm{d} t}\left[\left(R_{\theta} R_{t}\right) p\right]_{t=0}=\frac{\mathrm{d}}{\mathrm{d} t}\left[R_{t}\left(R_{\theta} p\right)\right]_{t=0}=V_{R_{\theta} p}
$$

e portanto

$$
\left(R_{\theta}^{*} \omega\right)_{p}\left(h+\lambda V_{p}\right)=\omega_{R_{\theta} p}\left(h^{\prime}+\lambda V_{R_{\theta} p}\right)=\lambda, \quad \forall h \in \mathcal{H}_{p}, \forall \lambda \in \mathbb{R}
$$

Portanto, $R_{\theta}^{*} \omega=\omega$ e então $\omega$ é forma de conexão.

A unicidade é simples: se $\omega$ e $\omega^{\prime}$ são duas formas de conexão tais que $\operatorname{ker} \omega=\operatorname{ker} \omega^{\prime}=$ $\mathcal{H}$, então necessariamente $\omega=f \omega^{\prime}$, onde $f: B(M) \rightarrow \mathbb{R}$ é função diferenciável. Mas então $f_{(p)}=f_{(p)} \omega^{\prime}\left(V_{p}\right)=\omega\left(V_{p}\right)=1, \forall p \in B(M)$. Portanto, $\omega=\omega^{\prime}$. 


\subsection{Transporte paralelo}

Veremos agora como uma conexão $\mathcal{H}$ em $B(M)$ nos fornece um certo meio de transportar vetores ao longo de curvas. Essa noção de "transporte paralelo" de vetores nos permitirá ainda uma interpretação geométrica da derivada de um campo em determinada direção.

Seja $M$ uma variedade sub-Riemanniana de contato , $\mathcal{H}$ uma conexão em $B(M)$ e $\alpha: I=[0,1] \rightarrow M$ uma curva diferenciável, regular. Considere então a imersão $\varphi: I \rightarrow$ $I \times M$ dada por $\varphi(t)=(t, \alpha(t))$. Seja então $\varphi^{*}(\alpha)=\left\{(t, v) \in I \times B(M): v \in \pi^{-1}(\alpha(t))\right\}$. É fácil ver que $\varphi^{*}(\alpha)$ é subvariedade bidimensional com bordo de $I \times B(M)$ (em verdade, $\varphi^{*}(\alpha)$ pode ser vista, localmente, como variedade produto de um aberto de $\left.\varphi(I) \operatorname{com} S^{1}\right)$. Considere então o seguinte campo definido em $\varphi^{*}(\alpha)$ :

$$
X_{(t, v)}=\left(\frac{\partial}{\partial t},\left(\left.\mathrm{~d} \pi_{v}\right|_{\mathcal{H}_{v}}\right)^{-1}(\dot{\alpha}(t))\right) \in T_{t} I \oplus T_{v} B(M) \cong T_{(t, v)}(I \times B(M)) .
$$

$X$ está bem definido, desde que $\left.\mathrm{d} \pi_{v}\right|_{\mathcal{H}_{v}}$ é isomorfismo e é evidente pela definição de $\varphi^{*}(\alpha)$, que $X_{(t, v)} \in T_{(t, v)} \varphi^{*}(\alpha)$. A diferenciabilidade de $X$ é verificável da seguinte maneira: dado $\left(t_{0}, v\right) \in \varphi^{*}(\alpha)$, existem campos diferenciáveis $X_{1} X_{2}, X_{3}$, definidos em uma vizinhança $W$ de $v$, que geram $\mathcal{H}$ nessa vizinhança. É claro então que, se $Y_{i}=\mathrm{d} \pi\left(X_{i}\right), Y_{1}, Y_{2}, Y_{3}$ serão campos diferenciáveis, definidos em uma vizinhança $U=\pi(W) \subset M$ de $\pi(v)=\alpha\left(t_{0}\right)$, que gerarão TM nessa vizinhança. É claro então que, numa vizinhança suficientemente pequena $\left.I_{t_{0}}=\right] t_{0}-\varepsilon, t_{0}+\varepsilon\left[, \dot{\alpha}(t)=\sum_{i=1}^{3} f(t)^{i} Y_{i}(\alpha(t))\right.$, onde $f^{i}: I_{t_{0}} \rightarrow \mathbb{R}$ são funções diferenciáveis. Mas note então que $\left.X\right|_{I_{t_{0}} \times W}$ vai ser dado por $X_{(t, v)}=\left(\frac{\partial}{\partial t}, \sum_{i=1}^{3} f^{i}(t) X_{i(t, v)}\right)$ e será portanto diferenciável.

Note que, se estendermos a ação de $S^{1}$ a $I \times B(M)$, definindo a ação agindo somente no segundo fator, isto é,

$$
R_{\theta}(t, v)=\left(t, R_{\theta} v\right)
$$

teremos que

$$
X_{\left(t, R_{\theta} v\right)}=\mathrm{d} R_{\theta}\left(X_{(t, v)}\right) .
$$

De fato, se $X_{(t, v)}=\left(\frac{\partial}{\partial t}, Y\right) \in T I \oplus T B(M)$, então $Y \in \mathcal{H}_{v}$ e $\mathrm{d} \pi(Y)=\dot{\alpha}(t)$. Ora, segue então que $\mathrm{d} R_{\theta}(Y) \in \mathcal{H}_{R_{\theta} v}$, pois $\mathcal{H}$ é conexão e $\mathrm{d} \pi\left(\mathrm{d} R_{\theta}(Y)\right)=\mathrm{d}\left(\pi \circ R_{\theta}(Y)\right)=\mathrm{d} \pi(Y)=$ 
$\dot{\alpha}(t)$. Portanto,

$$
X_{\left(t, R_{\theta} v\right)}=\left(\frac{\partial}{\partial t}, \mathrm{~d} R_{\theta}(Y)\right)=\mathrm{d} R_{\theta}\left(\frac{\partial}{\partial t}, Y\right)=\mathrm{d} R_{\theta}\left(X_{(t, v)}\right) .
$$

Seja então $v \in \pi^{-1}(\alpha(0))$. Segue do Teorema de Existência e Unicidade de soluções para equações diferenciais ordinárias de primeira ordem (ver [11], p.37) e de 2.3, que existe uma única curva $\tilde{\alpha}_{v}: I \rightarrow \varphi^{*}(\alpha)$ integral ao campo $X$, definido acima, tal que $\tilde{\alpha}_{v}(0)=(0, v)$.

Observe que (2.3) implica que, se $\widetilde{\alpha}_{v}$ é curva integral a $X$ tal que $\widetilde{\alpha}_{v}(0)=(0, v)$, $\tilde{\beta}=R_{\theta} \tilde{\alpha}_{v}$ será a (única) curva integral a $X$ tal que $\tilde{\beta}(0)=\left(0, R_{\theta} v\right)$. Em outras palavras, temos que $\tilde{\alpha}_{R_{\theta} v} \equiv R_{\theta} \tilde{\alpha}_{v}$. De fato, note que

$$
\dot{\tilde{\beta}}(t)=\mathrm{d} R_{\theta}\left(\dot{\tilde{\alpha}}_{v}(t)\right)=\mathrm{d} R_{\theta}\left(X_{\widetilde{\alpha}_{v}(t)}\right)=X_{R_{\theta} \widetilde{\alpha}_{v}(t)}=X_{\widetilde{\beta}(t)} .
$$

Definição 2.3 Sejam $M, \mathcal{H}, \alpha, v$ e $\widetilde{\alpha}_{v}$ como definidos acima. Se denotarmos então por $\pi_{2}: I \times B(M) \rightarrow B(M)$ a projeção no segundo fator, definimos o levantamento horizontal de $\alpha$ a partir de $v$ (relativo à conexáo $\mathcal{H}$ ) como sendo a curva $\ell_{(\alpha, v)}: I \rightarrow B(M)$, dada por $\ell_{(\alpha, v)}=\pi_{2} \circ \tilde{\alpha}_{v}$.

Da unicidade de $\widetilde{\alpha}_{v}$, é fácil concluir que $\ell_{(\alpha, v)}$ é a única curva em $B(M)$ tal que $\ell_{(\alpha, v)}(0)=v, \quad \pi \circ \ell_{(\alpha, v)} \equiv \alpha \quad$ e $\quad \dot{\ell}_{(\alpha, v)}(t) \in \mathcal{H}, \forall t \in I$.

A observação anterior então nos diz que

$$
\ell_{\left(\alpha, R_{\theta} v\right)} \equiv R_{\theta} \ell_{(\alpha, v)}
$$

Definição 2.4 Dados $M, \mathcal{H}$ e $\alpha$, como anteriormente, definimos o transporte paralelo de $v \in \pi^{-1}(\alpha(0))$ por $\alpha$ a $\alpha\left(t_{0}\right)$ como sendo $T P_{\alpha\left(t_{0}\right)}(v)=\ell_{(\alpha, v)}\left(t_{0}\right) \in \pi^{-1}\left(\alpha\left(t_{0}\right)\right)$, onde $\ell_{(\alpha, v)}$ é o levantamento horizontal de $\alpha$ a partir de $v$.

Segue da definição 2.4 que

$$
T P_{\alpha\left(t_{0}\right)}\left(R_{\theta} v\right)=\ell_{\left(\alpha, R_{\theta}(v)\right)}\left(t_{0}\right)=R_{\theta} \ell_{(\alpha, v)}\left(t_{0}\right)=R_{\theta} T P_{\alpha\left(t_{0}\right)}(v) .
$$


Estendamos então o transporte paralelo a $T_{\alpha(0)} M$ de forma a manter essa propriedade:

Definição 2.5 Se $\alpha$ é como na definição anterior e $Y \in T_{\alpha(0)} M, Y=\lambda_{1} v+\lambda_{2} \xi$, onde $v \in \pi^{-1}(\alpha(0))$ e $\xi$ é o campo fundamental, definimos o transporte paralelo de $Y$ por $\alpha$ a $\alpha\left(t_{0}\right)$ como sendo

$$
T P_{\alpha\left(t_{0}\right)}(Y)=\lambda_{1} T P_{\alpha\left(t_{0}\right)}(v)+\lambda_{2} \xi_{\alpha(t)} \in T_{\alpha\left(t_{0}\right)} M
$$

É imediato então que o transporte paralelo nos dará uma isometria entre $\mathcal{D}_{\alpha(0)}$ e $\mathcal{D}_{\alpha\left(t_{0}\right)}$ e que o campo $\xi$ é paralelo, isto é, $T P_{\alpha\left(t_{0}\right)}(\xi)=\xi_{\alpha\left(t_{0}\right)}$, qualquer que seja a curva $\alpha$.

Observação: Note agora que, se $\beta: I \rightarrow B(M)$ é um levantamento qualquer de $\alpha: I \rightarrow$ $M$, isto é, $\beta$ é tal que $\pi \circ \beta \equiv \alpha$, lançando mão da teoria de recobrimentos (ver [8], p.182), segue que existe $\theta: I \rightarrow \mathbb{R}$ diferenciável tal que $\beta \equiv R_{\theta(t)} \ell_{(\alpha, v)}$ e mais, se $\beta$ é tal que $\beta(0)=v$, existe uma única tal $\theta: I \rightarrow \mathbb{R} \operatorname{com} \theta(0)=0$.

Seja então $\beta=R_{\theta(t)} \ell_{(\alpha, v)}$, para alguma $\theta: I \rightarrow \mathbb{R}$ diferenciável com $\theta(0)=0$. Observe que

$$
\dot{\beta}(t)=\dot{\theta}(t) V_{\alpha(t)}+\mathrm{d}\left(R_{\theta(t)}\right)\left(\dot{\ell}_{(\alpha, v)}(t)\right) .
$$

Mas, por definição, $\dot{\ell}_{(\alpha, v)}(t) \in \mathcal{H}$ e, comod $R_{\theta}(\mathcal{H})=\mathcal{H}$, segue que $\mathrm{d}\left(R_{\theta(t)}\right)\left(\dot{\ell}_{(\alpha, v)}(t)\right) \in \mathcal{H}$ e portanto

$$
\omega(\dot{\beta}(t))=\dot{\theta}(t),
$$

onde $\omega$ é a forma de conexão associada a $\mathcal{H}$. Podemos então obter $\theta(t)$, se conhecido $\omega(\dot{\beta}(t)), \forall t \in[0,1]$,

$$
\theta(t)=\int_{0}^{t} \omega(\dot{\beta}(s)) \mathrm{d} s .
$$




\subsection{Equações de estrutura, forma da conexão sub- Riemanniana}

Considere a extensão da métrica $<,>$ a todo $T M$, realizada impondo-se que $\xi$ seja campo unitário e normal a $\mathcal{D}$. Sobre $B(M)$, definamos então as seguintes 1-formas: se $X \in$ $T_{v} B(M)$ :

$$
\begin{aligned}
\theta^{1}(X) & =\langle\mathrm{d} \pi(X), v\rangle \\
\theta^{2}(X) & =\langle\mathrm{d} \pi(X), i v\rangle \\
\theta^{3} & =\pi^{*} \theta,
\end{aligned}
$$

onde $i v$ denota apenas $R_{\frac{\pi}{2}} v$ e $\theta$ é a forma de contato (normalizada) de $M$.

Observe que outra forma de expressar $\theta^{3}$ seria

$$
\theta^{3}(X)=\langle\mathrm{d} \pi(X), \xi\rangle
$$

Se $\omega$ é uma forma de conexão em $B(M)$, repare que $\left(\omega, \theta^{i}\right), i=1,2,3$, é base de $T^{*} B(M)$. De fato, se $X \in T_{v} B(M)$ e $\omega(X)=\theta^{i}(X)=0$, teremos, de $\theta^{i}(X)=0$, que $\mathrm{d} \pi(X)=0$ e portanto $X=\lambda V_{v}$, mas de $\omega(X)=0$, temos que $\lambda=0$ e portanto $X=0$. Note ainda que, de (2.2), segue que $\theta^{i}(V)=0, i=1,2,3$.

Vejamos agora como se comportam as formas em relação à ação de $S^{1}$.

$$
\begin{aligned}
\left(R_{\alpha}^{*} \theta^{1}\right)_{v}(X) & =\theta_{R_{\alpha} v}^{1}\left(\mathrm{~d} R_{\alpha}(X)\right)=\left\langle\mathrm{d} \pi \circ \mathrm{d} R_{\alpha}(X), R_{\alpha} v\right\rangle \\
& =\langle\mathrm{d} \pi(X), \cos \alpha v+\operatorname{sen} \alpha i v\rangle \\
& =\cos \alpha \theta_{v}^{1}(X)+\operatorname{sen} \alpha \theta_{v}^{2}(X) .
\end{aligned}
$$

Calculando então de forma análoga $R_{\alpha}^{*} \theta^{2}$ e notando que $R_{\alpha}^{*} \theta^{3}=R_{\alpha}^{*} \pi^{*} \theta=\left(\pi \circ R_{\alpha}\right)^{*} \theta=$ $\pi^{*} \theta=\theta^{3}$, obtemos

$$
\begin{aligned}
& R_{\alpha}^{*} \theta^{1}=\cos \alpha \theta^{1}+\operatorname{sen} \alpha \theta^{2} \\
& R_{\alpha}^{*} \theta^{2}=-\operatorname{sen} \alpha \theta^{1}+\cos \alpha \theta^{2} \\
& R_{\alpha}^{*} \theta^{3}=\theta^{3} .
\end{aligned}
$$


Antes de obtermos as equações de estrutura, precisaremos demonstrar ainda o seguinte lema:

Lema 2.1 Se ф é uma 2-forma diferenciável em $B(M)$ que satisfaça as duas condições

(i) $R_{\theta}^{*} \phi=\phi$;

(ii) $\iota_{V} \phi=0$,

então $\phi$ é projetável, isto é, existe uma 2-forma $\varphi$ em $M$ tal que $\pi^{*} \varphi=\phi$. Além disso, tal $\varphi$ é única.

Prova: Construímos $\varphi$ da seguinte forma: dados $m \in M$ e $v_{1}, v_{2} \in T_{m} M$, defina

$$
\varphi_{(m)}\left(v_{1}, v_{2}\right)=\phi_{v}\left(X_{1}, X_{2}\right)
$$

onde $v \in \pi^{-1}(m)$ e $X_{i} \in T_{v} B(M)$, com $\mathrm{d} \pi\left(X_{i}\right)=v_{i}, i=1,2$. Precisamos mostrar então que $\varphi$ está bem definida. Note primeiro que $\phi_{v}\left(X_{1}, X_{2}\right)$ não depende da escolha de $X_{1} \mathrm{e}$ $X_{2}$. De fato, se $\widetilde{X}_{1}$ e $\widetilde{X}_{2}$ são vetores tais que $\widetilde{X}_{i} \in T_{v} B(M)$ e $\mathrm{d} \pi\left(\widetilde{X}_{i}\right)=v_{i}$, então

$$
\mathrm{d} \pi\left(X_{i}-\widetilde{X}_{i}\right)=\mathrm{d} \pi\left(X_{i}\right)-\mathrm{d} \pi\left(\widetilde{X}_{i}\right)=v-v=0,
$$

portanto, $\widetilde{X}_{i}-X_{i}=\lambda_{i} V_{v}$, onde $V$ é o campo vertical e, devido a (ii), temos

$$
\phi_{v}\left(\widetilde{X_{1}}, \widetilde{X}_{2}\right)=\phi_{v}\left(X_{1}+\lambda_{1} V, X_{2}+\lambda_{2} V\right)=\phi_{v}\left(X_{1}, X_{2}\right)
$$

Note agora que $\phi_{v}\left(X_{1}, X_{2}\right)$ não depende do elemento da fibra escolhido, pois se $\tilde{v} \epsilon$ $\pi^{-1}(m)$, então existe $R_{\theta}$ tal que $\tilde{v}=R_{\theta} v$. Tomando então $\widetilde{X}_{i}:=\mathrm{d} R_{\theta}\left(X_{i}\right)$, observe que $\mathrm{d} \pi\left(\widetilde{X}_{i}\right)=\mathrm{d} \pi \circ \mathrm{d} R_{\theta}\left(X_{i}\right)=\mathrm{d} \pi\left(X_{i}\right)=v_{i}$ e

$$
\phi_{\widetilde{v}}\left(\widetilde{X}_{1}, \widetilde{X}_{2}\right)=\phi_{R_{\theta} v}\left(\mathrm{~d} R_{\theta} X_{1}, \mathrm{~d} R_{\theta} X_{2}\right)=\left(R_{\theta}^{*} \phi\right)_{v}\left(X_{1}, X_{2}\right)=\phi_{v}\left(X_{1}, X_{2}\right)
$$

por (ii). Segue então que $\varphi$ está bem definida. A diferenciabilidade de $\varphi$ decorre da diferenciabilidade de $\phi$. Observe agora que, dada a forma como foi construída,

$$
\pi^{*} \varphi_{v}\left(X_{1}, X_{2}\right)=\varphi_{m}\left(\mathrm{~d} \pi X_{1}, \mathrm{~d} \pi X_{2}\right)=\phi_{(m, v)}\left(X_{1}, X_{2}\right)
$$


para todo $v \in B(M)$ e $X_{1}, X_{2} \in T_{v} B(M)$. Portanto, $\pi^{*} \varphi=\phi$.

Por fim, a unicidade decorre do fato de que, se $\varphi_{1}$ e $\varphi_{2}$ são tais que $\pi^{*} \varphi_{1}=\pi^{*} \varphi_{2}=$ $\phi$, então temos que $\pi^{*}\left(\varphi_{1}-\varphi_{2}\right)=0$ e, como $\pi$ é submersão, segue que $\pi^{*}$ é injetora, $\varphi_{1}-\varphi_{2}=0, \varphi_{1}=\varphi_{2}$.

Uma conseqüência desse lema é que $\mathrm{d} \omega$, assim também como $\theta^{1} \wedge \theta^{2}$, são projetáveis. De fato, $\iota_{V}\left(\theta^{1} \wedge \theta^{2}\right)=0 \mathrm{e}$

$$
\begin{aligned}
R_{\alpha}^{*}\left(\theta^{1} \wedge \theta^{2}\right) & =R_{\alpha}^{*} \theta^{1} \wedge R_{\alpha}^{*} \theta^{2} \\
& =\left(\cos \alpha \theta^{1}+\operatorname{sen} \alpha \theta^{2}\right) \wedge\left(-\operatorname{sen} \alpha \theta^{1}+\cos \alpha \theta^{2}\right) \\
& =\theta^{1} \wedge \theta^{2}
\end{aligned}
$$

e no caso de $d \omega$,

$$
R_{\theta}^{*}(\mathrm{~d} \omega)=\mathrm{d}\left(R_{\theta}^{*} \omega\right)=\mathrm{d} \omega
$$

e, se $\left\{V, E_{1}, E_{2}, E_{3}\right\}$ é a base dual de $\left\{\omega, \theta^{1}, \theta^{2}, \theta^{3}\right\}$,

$$
\begin{aligned}
\mathrm{d} \omega_{v}\left(V, E_{i}\right) & =-\omega_{v}\left(\left[V, E_{i}\right]\right) \\
& =\omega_{v}\left(L_{V} E_{i}\right)=\omega_{v}\left(\lim _{t \rightarrow 0} \frac{\mathrm{d}\left(\phi_{t}\right)^{-1} E_{i}(t)-E_{i}(0)}{t}\right),
\end{aligned}
$$

onde $\phi_{t}$ é o difeomorfismo local dado pelo fluxo de $V$, mas observe que, de (2.1), segue que o fluxo de $V$ é $\phi_{t}(v)=R_{t}(v), v \in B(M)$, portanto $\mathrm{d} \phi_{t}=\mathrm{d} R_{t}$. Da propriedade (a) da conexão, $\mathrm{d} R_{t}(\mathcal{H})=\mathcal{H}$ e $\mathrm{d} R_{t}^{-1}(\mathcal{H})=\mathcal{H}$. Como $E_{i}$ é seç̧ão de $\mathcal{H}$, para $i=1,2,3$, segue que a expressão dentro do limite é vetor pertencente a $\mathcal{H}$ e portanto

$$
\lim _{t \rightarrow 0} \frac{\mathrm{d}\left(R_{t}\right)^{-1} E_{i}(t)-E_{i}(0)}{t}=X \in \mathcal{H}_{v}
$$

e da continuidade de $\omega_{v}$, temos que $\mathrm{d} \omega_{v}\left(V, E_{i}\right)=\omega_{v}(X)=0$. Como $\mathrm{d} \omega(V, V)=0$, segue então que $\iota_{V} \mathrm{~d} \omega=0$. Observe que, como $\iota_{V} \mathrm{~d} \omega=0, \mathrm{~d} \omega$ se escreve da forma

$$
\mathrm{d} \omega=-K \theta^{1} \wedge \theta^{2}+W_{1} \theta^{1} \wedge \theta^{3}+W_{2} \theta^{2} \wedge \theta^{3}
$$

e que, como d $\omega$ e $\theta^{1} \wedge \theta^{2}$ são projetáveis, $K$ também é projetável, isto é, independe do elemento da fibra em que esteja sendo calculado. Note que a projetividade de $\mathrm{d} \omega$ ainda 
nos garante que o campo $W^{\#}=W_{1} \otimes E_{1}+W_{2} \otimes E_{2}$ é projetável. Confiramos: do fato de $\mathrm{d} \omega$ ser projetável, tiramos facilmente que, dado $v \in \pi^{-1}(m)$

$$
\begin{aligned}
& W_{1}(v)=\mathrm{d} \omega(v, \xi) \\
& W_{2}(v)=\mathrm{d} \omega(i v, \xi)
\end{aligned}
$$

(observe que $\omega$ aqui já não é a forma em $B(M)$, mas sim o "pull-back" da forma $\omega$ em $B(M)$ por qualquer seç̧ão $s$ tal que $s(m)=v$. Denotamos da mesma maneira para não acabar por sobrecarregar a notação). Veja que, se $\widetilde{v}=R_{\alpha} v$, então

$$
\begin{aligned}
W_{1}(\tilde{v}) & =\mathrm{d} \omega\left(R_{\alpha} v, \xi\right)=\cos \alpha W_{1}(v)+\operatorname{sen} \alpha W_{2}(v) \\
\text { e } \quad W_{2}(\tilde{v}) & =-\operatorname{sen} \alpha W_{1}(v)+\cos \alpha W_{2}(v) .
\end{aligned}
$$

Observe então que o campo (em $M)$

$$
\widetilde{W}_{(m)}^{\#}=W_{1}(v) v+W_{2}(v) i v \text {, onde } v \in \pi^{-1}(m),
$$

está bem definido, pois se $\widetilde{v}=R_{\alpha} v$,

$$
\begin{aligned}
W_{1}(\tilde{v}) \tilde{v}+W_{2}(\tilde{v}) i \tilde{v}= & \left(\cos \alpha W_{1}(v)+\operatorname{sen} \alpha W_{2}(v)\right)(\cos \alpha v+\operatorname{sen} \alpha i v) \\
& +\left(-\operatorname{sen} \alpha W_{1}(v)+\cos \alpha W_{2}(v)\right)(-\operatorname{sen} \alpha v+\cos \alpha i v) \\
= & W_{1}(v) v+W_{2}(v) i v .
\end{aligned}
$$

Resta-nos ver então que $\widetilde{W}^{\#}$ é projeção de $W^{\#}$, isto é, que o diagrama abaixo comuta:

$$
\begin{array}{ccc}
B(M) & \stackrel{W^{\#}}{\longrightarrow} & T B(M) \\
\downarrow \pi & & \downarrow \mathrm{d} \pi \\
M & \stackrel{\widetilde{W}^{\#}}{\longrightarrow} & T M
\end{array}
$$

mas,

$$
\begin{aligned}
\mathrm{d} \pi \circ W^{\#}(v) & =\mathrm{d} \pi\left(W_{1}(v) E_{1(v)}+W_{2}(v) E_{2(v)}\right) \\
& =W_{1}(v) v+W_{2}(v) i v=\widetilde{W}^{\#} \circ \pi(v) .
\end{aligned}
$$

Note que $W=W_{1} \theta^{1}+W_{2} \theta^{2}$ é tal que $W(v)=\left\langle W^{\#}, v\right\rangle$ e que $W$ é também projetável. 
Definição 2.6 A projeção de $K$ a $M$ é chamada de função curvatura de $M$.

Escrevamos agora $\mathrm{d} \theta^{1}$ e $\mathrm{d} \theta^{2}$ como combinação linear dos produtos dois a dois dos elementos da base $\left\{\omega, \theta^{i}\right\}, i=1,2,3$ :

$$
\begin{aligned}
& \mathrm{d} \theta^{1}=a_{01} \omega \wedge \theta^{1}+a_{02} \omega \wedge \theta^{2}+a_{03} \omega \wedge \theta^{3}+a_{12} \theta^{1} \wedge \theta^{2}+a_{13} \theta^{1} \wedge \theta^{3}+a_{23} \theta^{2} \wedge \theta^{3} \\
& \mathrm{~d} \theta^{2}=b_{01} \omega \wedge \theta^{1}+b_{02} \omega \wedge \theta^{2}+b_{03} \omega \wedge \theta^{3}+b_{12} \theta^{1} \wedge \theta^{2}+b_{13} \theta^{1} \wedge \theta^{3}+b_{23} \theta^{2} \wedge \theta^{3} .
\end{aligned}
$$

Seja $\left\{V, e_{i}\right\}$ a base dual de $\left\{\omega, \theta^{i}\right\}$, note que

$$
a_{01}=\mathrm{d} \theta^{1}\left(V, e_{1}\right)=-\theta^{1}\left(\left[V, e_{1}\right]\right)=-\theta^{1}\left(L_{V} e_{1}\right),
$$

basta portanto obter $L_{V} e_{1}$ para se conhecer $a_{01}$. Analogamente, basta sabermos $L_{V} e_{i}$, $i=1,2,3$, para obtermos $a_{0 i}$ e $b_{0 i}, i=1,2$. Mas sabemos que

$$
L_{V} e_{i}=\left.\frac{\mathrm{d}}{\mathrm{d} t}\left(\mathrm{~d} \phi_{-t}\left(e_{i}(t)\right)\right)\right|_{t=0}
$$

onde $\phi_{t}(v)=R_{t}(v)$. Agora, para conhecermos $\mathrm{d} R_{t}$, basta que dualizemos as relações que já conhecemos:

$$
\begin{aligned}
R_{t}^{*} \theta^{1} & =\cos t \theta^{1}+\operatorname{sen} t \theta^{2} \\
R_{t}^{*} \theta^{2} & =-\operatorname{sen} t \theta^{1}+\cos t \theta^{2} \\
R_{t}^{*} \theta^{3} & =\theta^{3} \\
R_{t}^{*} \omega & =\omega .
\end{aligned}
$$

Obteremos

$$
\begin{aligned}
\mathrm{d} R_{t}\left(e_{1}\right) & =\cos t e_{1}-\operatorname{sen} t e_{2} \\
\mathrm{~d} R_{t}\left(e_{2}\right) & =\operatorname{sente}_{1}+\cos t e_{2} \\
\mathrm{~d} R_{t}\left(e_{3}\right) & =e_{3} \\
\mathrm{~d} R_{t}(V) & =V .
\end{aligned}
$$

Portanto, teremos que

$$
\begin{aligned}
L_{V}\left(e_{1}\right) & =\left.\frac{\mathrm{d}}{\mathrm{d} t}\left(\cos t e_{1}+\operatorname{sen} t e_{2}\right)\right|_{t=0}=e_{2} \\
L_{V}\left(e_{2}\right) & =\left.\frac{\mathrm{d}}{\mathrm{d} t}\left(\operatorname{sen} t e_{1}-\cos t e_{2}\right)\right|_{t=0}=-e_{1} \\
L_{V}\left(e_{3}\right) & =\left.\frac{\mathrm{d}}{\mathrm{d} t}\left(e_{3}\right)\right|_{t=0}=0
\end{aligned}
$$


e então

$$
\begin{array}{ll}
a_{01}=-\theta^{1}\left(L_{V} e_{1}\right)=0 & b_{01}=-\theta^{2}\left(L_{V} e_{1}\right)=-1 \\
a_{02}=-\theta^{1}\left(L_{V} e_{2}\right)=1 & b_{02}=-\theta^{2}\left(L_{V} e_{2}\right)=0 \\
a_{03}=-\theta^{1}\left(L_{V} e_{3}\right)=0 & b_{03}=-\theta^{2}\left(L_{V} e_{3}\right)=0
\end{array}
$$

Agora, fazendo o produto e a soma apropriados,

$$
\mathrm{d} \theta^{1} \wedge \theta^{2}-\theta^{1} \wedge \mathrm{d} \theta^{2}=\left(-a_{13}-b_{23}\right) \theta^{1} \wedge \theta^{2} \wedge \theta^{3}
$$

Mas $\mathrm{d} \theta^{1} \wedge \theta^{2}-\theta^{2} \wedge \mathrm{d} \theta^{2}=\mathrm{d}\left(\theta^{1} \wedge \theta^{2}\right)$ e observe que já sabemos que $\theta^{1} \wedge \theta^{2}$ é projetável. É simples verificar que a projeção de $\theta^{1} \wedge \theta^{2}$ é exatamente a forma volume (ou elemento de área) $\mathrm{d} V$ e portanto

$$
-\left(a_{13}+b_{23}\right) \theta^{1} \wedge \theta^{2} \wedge \theta^{3}=\mathrm{d}\left(\theta^{1} \wedge \theta^{2}\right)=\mathrm{d}\left(\pi^{*} \mathrm{~d} V\right)=\frac{1}{2} d\left(\pi^{*} \mathrm{~d} \theta\right)=\frac{1}{2} \pi^{*} \mathrm{~d}(\mathrm{~d} \theta)=\frac{1}{2} \pi^{*}(0)=0 .
$$

Portanto, $a_{13}=-b_{23}$.

As equações então ficam da seguinte forma:

$$
\left\{\begin{array}{l}
\mathrm{d} \theta^{1}-\omega \wedge \theta^{2}=a \theta^{1} \wedge \theta^{3}+b \theta^{2} \wedge \theta^{3}+f \theta^{1} \wedge \theta^{2} \\
\mathrm{~d} \theta^{2}+\omega \wedge \theta^{2}=c \theta^{1} \wedge \theta^{3}-a \theta^{2} \wedge \theta^{3}+g \theta^{1} \wedge \theta^{2}
\end{array}\right.
$$

Essas equações são chamadas primeiras equações de estrutura da geometria sub-riemanniana, enquanto a equação (2.7) é dita segunda equação de estrutura.

A seguir, demonstramos o teorema de existência e unicidade da forma de conexão sub-Riemanniana, cuja generalização para dimensões superiores encontra-se em [6].

Teorema 2.1 Dada uma variedade sub-Riemanniana de contato de $\operatorname{dim}=3$, orientada, existe uma única forma de conexão tal que $f=g=0$ e $b=c$.

Prova: Seja $\omega$ uma forma de conexão qualquer em $B(M)$ cujas equações de estrutura sejam como (2.8). Suponha $\tilde{\omega}$ outra forma de conexão, como $(\omega-\widetilde{\omega})(V)=0$, segue que $\omega-\tilde{\omega}$ pertence ao subespaço gerado por $\theta^{1}, \theta^{2}, \theta^{3}$, ou melhor,

$$
\omega=\widetilde{\omega}+\alpha \theta^{1}+\beta \theta^{2}+\gamma \theta^{3} .
$$


Substituindo $\omega$ em (2.8), obtemos

$$
\begin{aligned}
& \mathrm{d} \theta^{1}-\tilde{\omega} \wedge \theta^{2}=a \theta^{1} \wedge \theta^{3}+(b-\gamma) \theta^{2} \wedge \theta^{3}+(f+\alpha) \theta^{1} \wedge \theta^{2} \\
& \mathrm{~d} \theta^{2}+\widetilde{\omega} \wedge \theta^{1}=(c+\gamma) \theta^{1} \wedge \theta^{3}-a \theta^{2} \wedge \theta^{3}+(g+\beta) \theta^{1} \wedge \theta^{2} .
\end{aligned}
$$

Portanto, a unicidade de tal forma será conseqüência da unicidade da solução do sistema

$$
\left\{\begin{array} { l } 
{ \alpha = - f } \\
{ \beta = - g } \\
{ b - \gamma = c + \gamma }
\end{array} \quad \Rightarrow \quad \left\{\begin{array}{l}
\alpha=-f \\
\beta=-g \\
\gamma=\frac{b-c}{2}
\end{array}\right.\right.
$$

donde $\widetilde{\omega}=\omega+f \theta^{1}+g \theta^{2}+\frac{c-b}{2} \theta^{3}$. Resta-nos mostrar então que a forma $\widetilde{\omega}$ assim construída é forma de conexão.

i) É claro que $\widetilde{\omega}(V)=\omega(V)=1$.

ii) Mostremos então que $R_{\alpha}^{*} \tilde{\omega}=\tilde{\omega}$.

$$
\begin{aligned}
R_{\alpha}^{*} \widetilde{\omega}= & R_{\alpha}^{*} \omega+R_{\alpha}^{*}(f) \cdot R_{\alpha}^{*} \theta^{1}+R_{\alpha}^{*}(g) R_{\alpha}^{*} \theta^{2}+\frac{1}{2} R_{\alpha}^{*}(c-b) R_{\alpha}^{*} \theta^{3} \\
= & \omega+f \circ R_{\alpha}\left(\cos \alpha \theta^{1}+\operatorname{sen} \alpha \theta^{2}\right)+g \circ R_{\alpha}\left(-\operatorname{sen} \alpha \theta^{1}+\cos \alpha \theta^{2}\right) \\
& +\frac{1}{2}\left(c \circ R_{\alpha}-b \circ R_{\alpha}\right) \theta^{3} \\
= & \omega+\left(\cos \alpha \cdot f \circ R_{\alpha}-\operatorname{sen} \alpha \cdot g \circ R_{\alpha}\right) \theta^{1}+\left(\operatorname{sen} \alpha \cdot f \circ R_{\alpha}+\cos \alpha \cdot g \circ R_{\alpha}\right) \theta^{2} \\
& +\frac{1}{2}\left(c \circ R_{\alpha}-b \circ R_{\alpha}\right) .
\end{aligned}
$$

Agora, observe que

$$
\begin{aligned}
f \circ R_{\alpha_{[v]}} & =f\left(R_{\alpha} v\right)=\mathrm{d} \theta_{R_{\alpha} v}^{1}\left(e_{1}, e_{2}\right)=\left(R_{\alpha}^{*} \mathrm{~d} \theta^{1}\right)_{v}\left(\mathrm{~d} R_{\alpha}^{-1} e_{1}, \mathrm{~d} R_{\alpha}^{-1} e_{2}\right) \\
& =\left(\cos \alpha \mathrm{d} \theta^{1}+\operatorname{sen} \alpha \mathrm{d} \theta^{2}\right)_{v}\left(\cos \alpha e_{1}-\operatorname{sen} \alpha e_{2}, \operatorname{sen} \alpha e_{1}+\cos \alpha e_{2}\right) \\
& =\cos \alpha \mathrm{d} \theta_{v}^{1}\left(e_{1}, e_{2}\right)+\operatorname{sen} \alpha \mathrm{d} \theta^{2}\left(e_{1}, e_{2}\right) \\
& =\cos \alpha f_{(v)}+\operatorname{sen} \alpha g_{(v)} .
\end{aligned}
$$

Portanto,

$$
f \circ R_{\alpha}=\cos \alpha f+\operatorname{sen} \alpha g
$$


e, calculando de forma análoga, obtemos

$$
\begin{aligned}
& g \circ R_{\alpha}=-\operatorname{sen} \alpha f+\cos \alpha g \\
& c \circ R_{\alpha}=-2 \operatorname{sen} \alpha \cos \alpha a-\operatorname{sen}^{2} \alpha b+\cos ^{2} \alpha c \\
& b \circ R_{\alpha}=-2 \operatorname{sen} \alpha \cos \alpha a+\cos ^{2} \alpha b-\operatorname{sen}^{2} \alpha c
\end{aligned}
$$

e substituindo na expressão de $R_{\alpha}^{*} \tilde{\omega}$, obtemos

$$
R_{\alpha}^{*} \tilde{\omega}=\omega+f \theta^{1}+g \theta^{2}+\frac{c-b}{2} \theta^{3}=\widetilde{\omega}
$$

o que conclui a demonstração da proposição.

Definição 2.7 A forma de conexão que goza de tal propriedade é dita forma de conexão sub-Riemanniana.

Juntando então às três equações uma quarta, fácil de verificar, e sendo $\omega$ a forma da conexão sub-Riemanniana, teremos

$$
\left\{\begin{array}{l}
\mathrm{d} \omega=-K \theta^{1} \wedge \theta^{2}+W \wedge \theta^{3} \\
\mathrm{~d} \theta^{1}=\omega \wedge \theta^{2}+\tau^{1} \wedge \theta^{3} \\
\mathrm{~d} \theta^{2}=-\omega \wedge \theta^{1}+\tau^{2} \wedge \theta^{3} \\
\mathrm{~d} \theta^{3}=2 \theta^{1} \wedge \theta^{2}
\end{array}\right.
$$

onde

$$
W=W_{1} \theta^{1}+W_{2} \theta^{2}, \quad \tau^{1}=a \theta^{1}+b \theta^{2} \quad \text { e } \quad \tau^{2}=b \theta^{1}-a \theta^{2} .
$$

Proposição $2.2 O$ tensor $\tau=\tau^{1} \otimes E_{1}+\tau^{2} \otimes E_{2}$ é projetável sobre $M$.

Prova: Denotaremos a projeção de $\tau$ pela mesma letra: se $v \in T_{m} M$, definamos

$$
\tau_{(m)}(v)=\tau_{(e)}^{1}(X) \cdot e+\tau_{(e)}^{2}(X) \cdot i e,
$$

onde $e \in \pi^{-1}(m)$ e $X \in T_{e} B(M)$ é tal que $\pi(X)=v$. Precisamos mostrar que esse tensor está bem definido, isto é, que o segundo membro da equação não depende das escolhas de e e $X$. 
Note primeiro que independe da escolha de $X$, pois se $\widetilde{X}$ é tal que $\mathrm{d} \pi(\widetilde{X})$, segue de $\mathrm{d} \pi(\widetilde{X}-X)=0$ e portanto $\widetilde{X}=X+\lambda V$ e, pela definição de $\tau^{1}$ e $\tau^{2}$ é direto que $\tau^{i}(\widetilde{X})=\tau^{i}(X), i=1,2$. Seja agora $\tilde{e} \in \pi_{(m)}^{-1}$, é claro que $\tilde{e}=R_{\alpha} e$ para algum $R_{\alpha} \in S^{1}$ e, se $X \in T_{e} B(M)$ é tal que $\mathrm{d} \pi(X)=v$, note que $\mathrm{d} \pi\left(\mathrm{d} R_{\alpha}(X)\right)=v$. Tomemos então $\widetilde{X}=\mathrm{d} R_{\alpha} X$. Precisamos mostrar então que

$$
\tau_{(\widetilde{e})}^{1}(\widetilde{X}) \widetilde{e}+\tau_{(\widetilde{e})}^{2}(\widetilde{X}) i \widetilde{e}=\tau_{(e)}^{1}(X) e+\tau_{(e)}^{2}(X) i e
$$

Fazendo o "pull-back" por $R_{\alpha}^{*}$ das primeiras equações de estrutura, obtemos:

$$
\left\{\begin{array}{c}
\cos \alpha \mathrm{d} \theta^{1}+\operatorname{sen} \alpha \mathrm{d} \theta^{2}=-\operatorname{sen} \alpha \omega \wedge \theta^{1}+\cos \alpha \omega \wedge \theta^{2}+\left(a \circ R_{\alpha} \cos \alpha-b \circ R_{\alpha} \operatorname{sen} \alpha\right) \theta^{1} \wedge \theta^{3} \\
\quad+\left(a \circ R_{\alpha} \operatorname{sen} \alpha+b \circ R_{\alpha} \cos \alpha\right) \theta^{2} \wedge \theta^{3} \\
\quad \operatorname{sen} \alpha \mathrm{d} \theta^{1}+\cos \alpha \mathrm{d} \theta^{2}=-\cos \alpha \omega \wedge \theta^{1}-\operatorname{sen} \alpha \omega \wedge \theta^{2}+\left(b \circ R_{\alpha} \cos \alpha+a \circ R_{\alpha} \operatorname{sen} \alpha\right) \theta^{1} \wedge \theta^{3} \\
\quad+\left(-a \circ R_{\alpha} \cos \alpha+b \circ R_{\alpha} \operatorname{sen} \alpha\right) \theta^{2} \wedge \theta^{3}
\end{array}\right.
$$

e obtemos desse sistema, facilmente,

$$
\begin{aligned}
\mathrm{d} \theta^{1}= & \omega \wedge \theta^{2}+\left(\cos 2 \alpha \cdot a \circ R_{\alpha}-\operatorname{sen} 2 \alpha \cdot b \circ R_{\alpha}\right) \theta^{1} \wedge \theta^{3} \\
& +\left(\operatorname{sen} 2 \alpha \cdot a \circ R_{\alpha}+\cos 2 \alpha \cdot b \circ R_{\alpha}\right) \theta^{2} \wedge \theta^{3}
\end{aligned}
$$

e então teremos, necessariamente,

$$
\left\{\begin{array}{l}
\cos 2 \alpha . a \circ R_{\alpha}-\operatorname{sen} 2 \alpha \cdot b \circ R_{\alpha}=a \\
\operatorname{sen} 2 \alpha . a \circ R_{\alpha}+\cos 2 \alpha . b \circ R_{\alpha}=b
\end{array}\right.
$$

e

$$
\left\{\begin{array}{l}
a \circ R_{\alpha}=\cos 2 \alpha \cdot a+\operatorname{sen} 2 \alpha \cdot b \\
b \circ R_{\alpha}=-\operatorname{sen} 2 \alpha \cdot a+\cos 2 \alpha \cdot b
\end{array}\right.
$$

Agora

$$
\begin{aligned}
\tau_{(\widetilde{e})}^{1}(\widetilde{X}) & =a_{(\widetilde{e})} \circ \theta_{\widetilde{e}}^{1}(\widetilde{X})+b_{(\widetilde{e}} \theta_{\widetilde{e}}^{2}(\widetilde{X}) \\
& =a \circ R_{\alpha(e)}\left(R_{\alpha}^{*} \theta^{1}\right)_{(e)}(X)+b \circ R_{\alpha(e)}\left(R_{\alpha}^{*} \theta^{2}\right)_{e}(X) .
\end{aligned}
$$

Substituindo então $a \circ R_{\alpha(e)}, R_{\alpha}^{*} \theta^{1}$ e $R_{\alpha}^{*} \theta^{2}$ e fazendo as contas, obtemos

$$
\tau_{(\widetilde{e})}^{1}(\widetilde{X})=\left[\cos \alpha \cdot a_{(e)}+\operatorname{sen} \alpha \cdot b_{(e)}\right] \theta^{1}(X)+\left[-\operatorname{sen} \alpha \cdot a_{(e)}+\cos \alpha \cdot b_{(e)}\right] \theta^{2}(X)
$$

e, de forma análoga,

$$
\tau_{(\widetilde{e})}^{2}(\widetilde{X})=\left[-\operatorname{sen} \alpha \cdot a_{(e)}+\cos \alpha \cdot b_{(e)}\right] \theta^{1}(X)+\left[-\cos \alpha \cdot a_{(e)}-\operatorname{sen} \alpha \cdot b_{(e)}\right] \theta^{2}(X) .
$$


Agora, sabemos que

$$
\begin{aligned}
\tilde{e} & =\cos \alpha e+\operatorname{sen} \alpha i e \\
i \widetilde{e} & =-\operatorname{sen} \alpha e+\cos \alpha i e
\end{aligned}
$$

e, fazendo então as substituições e todos os cálculos, obtemos

$$
\begin{aligned}
& \tau_{(\widetilde{e})}^{1}(\widetilde{X}) \widetilde{e}+\tau_{(\widetilde{e})}^{2}(\widetilde{X}) i \widetilde{e} \\
& =\left[a_{(e)} \theta^{1}(X)+b_{(e)} \theta^{2}(X)\right] e+\left[b_{(e)} \theta^{1}(X)-a_{(e)} \theta^{2}(X)\right] \\
& =\tau_{e}^{1}(X) e+\tau_{e}^{2}(X) i e
\end{aligned}
$$

exatamente o que queríamos mostrar. Segue então que $\tau$, em $M$, está bem definida e é evidente, pela forma como foi construída, que esse tensor é a projeção do tensor $\tau$ em $B(M)$.

É interessante notar nesse ponto uma outra forma de se enunciar o teorema 2.1, em termos de torsão: existe uma única forma de conexão tal que a torsão (Riemanniana) $T$ satisfaça às seguintes propriedades:

i) $T(X, Y)$ é paralelo a $\xi, \forall X, Y \in \mathcal{D}$;

ii) A restrição a $\mathcal{D}$ da contração de $T$ na direção $\xi$ (que será então o nosso $\tau$ ) é um tensor simétrico.

Um outro enunciado em termos da torsão e da derivada covariante será enunciado mais adiante na seção 3.1 .

\subsection{As identidades de Bianchi}

Diferenciando as primeiras equações de estrutura, relativas à conexão sub-Riemanniana, obtemos as seguintes equações, conhecidas como primeiras identidades de Bianchi:

$$
\left[\mathrm{d} \tau^{1}-W \wedge \theta^{2}-\omega \wedge \tau^{2}\right] \wedge \theta^{3}=0
$$




$$
\begin{aligned}
& {\left[\mathrm{d} \tau^{2}+W \wedge \theta^{1}+\omega \wedge \tau^{1}\right] \wedge \theta^{3}=0} \\
& \mathrm{~d} \tau^{1} \wedge \theta^{2}-\theta^{1} \wedge \mathrm{d} \theta^{2}=0
\end{aligned}
$$

onde $\omega$ é a forma de conexão sub-Riemanniana. Observe que a terceira equação se trivializa e as outras duas, substituindo as expressões de $W, \mathrm{~d} \tau^{1}$ e $\mathrm{d} \tau^{2}$, podem ser escritas

$$
\begin{aligned}
& {\left[\mathrm{d} a \wedge \theta^{1}+\mathrm{d} b \wedge \theta^{2}-W_{1} \theta^{1} \wedge \theta^{2}-2 \omega \wedge \tau^{2}\right] \wedge \theta^{3}=0} \\
& {\left[\mathrm{~d} b \wedge \theta^{1}-\mathrm{d} a \wedge \theta^{2}-W_{2} \theta^{1} \wedge \theta^{2}+2 \omega \wedge \tau^{1}\right] \wedge \theta^{3}=0}
\end{aligned}
$$

A segunda identidade de Bianchi obtém-se diferenciando a segunda equação de estrutura:

$$
-\mathrm{d} K \wedge \theta^{1} \wedge \theta^{2}+K\left(\tau^{1} \wedge \theta^{2}+\theta^{1} \wedge \tau^{2}\right) \wedge \theta^{3}+\mathrm{d} W \wedge \theta^{3}=0
$$

ou, desenvolvendo $\tau^{1}, \tau^{2}$ e $\mathrm{d} W$,

$$
-\mathrm{d} K \wedge \theta^{1} \wedge \theta^{2}+\left[\left(\mathrm{d} W_{1}-W_{2} \omega\right) \wedge \theta^{1}+\left(\mathrm{d} W_{2}+W_{1} \omega\right) \wedge \theta^{2}\right] \wedge \theta^{3}=0 .
$$

\subsection{Exemplos}

(1) Espaço de Heisenberg, $H^{3}$.

Retomando o exemplo 1 da seção 1.2 , temos que

$$
\left\{\begin{array}{l}
\theta^{1}=\mathrm{d} x \\
\theta^{2}=\mathrm{d} y \\
\theta^{3}=\theta=-y \mathrm{~d} x+x \mathrm{~d} y-\mathrm{d} z
\end{array}\right.
$$

onde $\theta^{1}, \theta^{2}$ e $\theta^{3}$ acabam sendo, em verdade, o "pull-back" das formas fundamentais de $B\left(H^{3}\right)$ pela seção (global) $e_{1}=\frac{\partial}{\partial x}-y \frac{\partial}{\partial z}$.

Suponha $\omega=\Gamma_{1} \theta^{1}+\Gamma_{2} \theta^{2}+\Gamma_{3} \theta^{3}$, forma de conexão sub-Riemanniana. Determinemola, fazendo uso das primeiras equações de estrutura:

$$
\begin{aligned}
\mathrm{d} \theta^{1}-\omega \wedge \theta^{2} & =\mathrm{d}(\mathrm{d} x)-\Gamma_{1} \theta^{1} \wedge \theta^{2}-\Gamma_{3} \theta^{3} \wedge \theta^{2} \\
& =-\Gamma_{1} \theta^{1} \wedge \theta^{2}+\Gamma_{3} \theta^{2} \wedge \theta^{3} \\
\mathrm{~d} \theta^{2}+\omega \wedge \theta^{1} & =\mathrm{d}(\mathrm{d} y)+\Gamma_{2} \theta^{2} \wedge \theta^{1}+\Gamma_{3} \theta^{3} \wedge \theta^{1} \\
& =-\Gamma_{2} \theta^{1} \wedge \theta^{2}-\Gamma_{3} \theta^{1} \wedge \theta^{3}
\end{aligned}
$$


então a forma de conexão sub-Riemanniana é dada por $-\Gamma_{1}=-\Gamma_{2}=0$ e $\Gamma_{3}=-\Gamma_{3}$, ou seja, $\Gamma_{1}=\Gamma_{2}=\Gamma_{3}=0$. Portanto, $\omega=0$ é a forma de conexão sub-Riemanniana. Evidentemente o tensor $\tau$ associado à forma $\omega=0$ é nulo e, utilizando a segunda equação de estrutura,

$$
0=\mathrm{d}(0)=\mathrm{d} \omega=-K \theta^{1} \wedge \theta^{2}+W \wedge \theta^{3}
$$

concluímos que

$$
K=W_{1}=W_{2}=0 .
$$

(2) Esfera $S^{3}$

Na seção 1.2 , obtivemos

$$
\begin{aligned}
& \left\{\begin{array}{l}
e_{1}=-x_{3} \frac{\partial}{\partial x_{1}}+x_{4} \frac{\partial}{\partial x_{2}}+x_{1} \frac{\partial}{\partial x_{3}}-x_{2} \frac{\partial}{\partial x_{4}} \\
e_{2}=-x_{4} \frac{\partial}{\partial x_{1}}-x_{3} \frac{\partial}{\partial x_{2}}+x_{2} \frac{\partial}{\partial x_{3}}+x_{1} \frac{\partial}{\partial x_{4}} \\
e_{3}=-x_{2} \frac{\partial}{\partial x_{1}}+x_{1} \frac{\partial}{\partial x_{2}}-x_{4} \frac{\partial}{\partial x_{3}}+x_{3} \frac{\partial}{\partial x_{4}}
\end{array}\right. \\
& \left\{\begin{array}{l}
\theta^{1}=-x_{3} \mathrm{~d} x_{1}+x_{4} \mathrm{~d} x_{2}+x_{1} \mathrm{~d} x_{3}-x_{2} \mathrm{~d} x_{4} \\
\theta^{2}=-x_{4} \mathrm{~d} x_{1}-x_{3} \mathrm{~d} x_{2}+x_{2} \mathrm{~d} x_{3}+x_{1} \mathrm{~d} x_{4} \\
\theta^{3}=-x_{2} \mathrm{~d} x_{1}+x_{1} \mathrm{~d} x_{2}-x_{4} \mathrm{~d} x_{3}+x_{3} \mathrm{~d} x_{4}
\end{array}\right.
\end{aligned}
$$

onde essas formas (na verdade, a restrição delas a $S^{3}$ ) são nada mais que o "pullback" das formas fundamentais de $B\left(S^{3}\right)$ pela seção $e_{1}=-x_{3} \frac{\partial}{\partial x_{1}}+x_{4} \frac{\partial}{\partial x_{2}}+x_{1} \frac{\partial}{\partial x_{3}}-$ $x_{2} \frac{\partial}{\partial x_{4}}$. Temos então que, se $\omega=\Gamma_{1} \theta^{1}+\Gamma_{2} \theta^{2}+\Gamma_{3} \theta^{3}$,

$$
\begin{aligned}
\mathrm{d} \theta^{1}-\omega \wedge \theta^{2} & =2\left(\mathrm{~d} x_{1} \wedge \mathrm{d} x_{3}-\mathrm{d} x_{2} \wedge \mathrm{d} x_{4}\right)-\Gamma_{1} \theta^{1} \wedge \theta^{2}+\Gamma_{3} \theta^{2} \wedge \theta^{3} \\
\mathrm{~d} \theta^{2}+\omega \wedge \theta^{1} & =2\left(\mathrm{~d} x_{1} \wedge \mathrm{d} x_{4}+\mathrm{d} x_{2} \wedge \mathrm{d} x_{3}\right)-\Gamma_{2} \theta^{1} \wedge \theta^{2}-\Gamma_{3} \theta^{1} \wedge \theta^{3}
\end{aligned}
$$

e, se $\omega$ é forma de conexão sub-Riemanniana, temos necessariamente que

$$
\left\{\begin{array}{l}
\left(\mathrm{d} \theta^{1}-\omega \wedge \theta^{2}\right)\left(e_{1}, e_{2}\right)=\left(\mathrm{d} \theta^{2}+\omega \wedge \theta^{1}\right)\left(e_{1}, e_{2}\right)=0 \\
\left(\mathrm{~d} \theta^{1}-\omega \wedge \theta^{2}\right)\left(e_{2}, e_{3}\right)=\left(\mathrm{d} \theta^{2}+\omega \wedge \theta^{1}\right)\left(e_{1}, e_{3}\right)
\end{array}\right.
$$

mas,

$$
\left\{\begin{array}{l}
\left(\mathrm{d} \theta^{1}-\omega \wedge \theta^{2}\right)\left(e_{1}, e_{2}\right)=-\Gamma_{1} \\
\left(\mathrm{~d} \theta^{2}-\omega \wedge \theta^{1}\right)\left(e_{1}, e_{2}\right)=-\Gamma_{2} \\
\left(\mathrm{~d} \theta^{1}-\omega \wedge \theta^{2}\right)\left(e_{2}, e_{3}\right)=2+\Gamma_{3} \\
\left(\mathrm{~d} \theta^{2}-\omega \wedge \theta^{1}\right)\left(e_{1}, e_{3}\right)=-2-\Gamma_{3}
\end{array}\right.
$$


de onde temos que

$$
\Gamma_{1}=\Gamma_{2}=0 \quad \text { e } \quad \Gamma_{3}=-2
$$

ou seja, a forma de conexão sub-Riemanniana da nossa esfera é

$$
\omega=-2 \theta^{3} .
$$

Observe então que

$$
\mathrm{d} \omega=-4 \theta^{1} \wedge \theta^{2},
$$

de onde concluímos que a curvatura dessa nossa esfera é constante e igual a 4 e $W=0$. Por fim, percebendo ainda que

$$
\begin{aligned}
& \left(\mathrm{d} \theta^{1}-\omega \wedge \theta^{2}\right)\left(e_{1}, e_{3}\right)=0 \\
& \left(\mathrm{~d} \theta^{1}-\omega \wedge \theta^{2}\right)\left(e_{2}, e_{3}\right)=2+\Gamma_{3}=0 \\
& \left(\mathrm{~d} \theta^{2}-\omega \wedge \theta^{1}\right)\left(e_{1}, e_{3}\right)=-2-\Gamma_{3}=0 \\
& \left(\mathrm{~d} \theta^{2}+\omega \wedge \theta^{1}\right)\left(e_{1}, e_{3}\right)=0
\end{aligned}
$$

concluímos que o tensor $\tau=0$.

(3) Quádrica $Q^{3}$

Da seção 1.2 , temos que

$$
\begin{aligned}
& \left\{\begin{array}{l}
e_{1}=x_{3} \frac{\partial}{\partial x_{1}}-x_{4} \frac{\partial}{\partial x_{2}}+x_{1} \frac{\partial}{\partial x_{3}}-x_{2} \frac{\partial}{\partial x_{4}} \\
e_{2}=x_{4} \frac{\partial}{\partial x_{1}}+x_{3} \frac{\partial}{\partial x_{2}}+x_{2} \frac{\partial}{\partial x_{3}}+x_{1} \frac{\partial}{\partial x_{4}} \\
e_{3}=x_{2} \frac{\partial}{\partial x_{1}}-x_{1} \frac{\partial}{\partial x_{2}}+x_{4} \frac{\partial}{\partial x_{3}}-x_{3} \frac{\partial}{\partial x_{4}}
\end{array}\right. \\
& \left\{\begin{array}{l}
\theta^{1}=-x_{3} \mathrm{~d} x_{1}+x_{4} \mathrm{~d} x_{2}+x_{1} \mathrm{~d} x_{3}-x_{2} \mathrm{~d} x_{4} \\
\theta^{2}=-x_{4} \mathrm{~d} x_{1}-x_{3} \mathrm{~d} x_{2}+x_{2} \mathrm{~d} x_{3}+x_{1} \mathrm{~d} x_{4} \\
\theta^{3}=x_{2} \mathrm{~d} x_{1}-x_{1} \mathrm{~d} x_{2}-x_{4} \mathrm{~d} x_{3}+x_{3} \mathrm{~d} x_{4}
\end{array}\right.
\end{aligned}
$$

Se $\omega=\Gamma_{1} \theta^{1}+\Gamma_{2} \theta^{2}+\Gamma_{3} \theta^{3}$, temos que

$$
\begin{aligned}
& \mathrm{d} \theta^{1}-\omega \wedge \theta^{2}=2\left(\mathrm{~d} x_{1} \wedge \mathrm{d} x_{3}-\mathrm{d} x_{2} \wedge \mathrm{d} x_{4}\right)-\Gamma_{1} \theta^{1} \wedge \theta^{2}+\Gamma_{3} \theta^{2} \wedge \theta^{3} \\
& \mathrm{~d} \theta^{2}+\omega \wedge \theta^{1}=2\left(\mathrm{~d} x_{1} \wedge \mathrm{d} x_{4}+\mathrm{d} x_{2} \wedge \mathrm{d} x_{3}\right)-\Gamma_{2} \theta^{1} \wedge \theta^{2}-\Gamma_{3} \theta^{1} \wedge \theta^{3}
\end{aligned}
$$


daí,

$$
\begin{aligned}
& \left(\mathrm{d} \theta^{1}-\omega \wedge \theta^{2}\right)\left(e_{1}, e_{2}\right)=-\Gamma_{1} \\
& \left(\mathrm{~d} \theta^{1}-\omega \wedge \theta^{2}\right)\left(e_{1}, e_{3}\right)=0 \\
& \left(\mathrm{~d} \theta^{1}-\omega \wedge \theta^{2}\right)\left(e_{2}, e_{3}\right)=2\left(-x_{1}^{2}-x_{2}^{2}+x_{3}^{2}+x_{4}^{2}\right)+\Gamma_{3}=\Gamma_{3}-2 \\
& \left(\mathrm{~d} \theta^{2}+\omega \wedge \theta^{1}\right)\left(e_{1}, e_{2}\right)=-\Gamma_{2} \\
& \left(\mathrm{~d} \theta^{2}+\omega \wedge \theta^{1}\right)\left(e_{1}, e_{3}\right)=2\left(x_{1}^{2}+x_{2}^{2}-x_{3}^{2}-x_{4}^{2}\right)-\Gamma_{3}=2-\Gamma_{3} \\
& \left(\mathrm{~d} \theta^{2}+\omega \wedge \theta^{1}\right)\left(e_{1}, e_{3}\right)=0
\end{aligned}
$$

e, das condições $(*)$, obtemos que

$$
\Gamma_{1}=\Gamma_{2}=0 \quad \text { e } \quad \Gamma_{3}=2
$$

e portanto

$$
\omega=2 \theta^{3} \quad \text { e } \quad W=0
$$

e, da segunda equação de estrutura,

$$
\mathrm{d} \omega=4 \mathrm{~d} \theta^{3},
$$

concluímos que $K=-4$ e que $\tau=0$.

Observação: Não é difícil verificar agora que, se multiplicarmos a métrica da nossa variedade sub-Riemanniana de contato por uma constante $c$, obteremos as relações abaixo, entre as novas formas (fundamentais e de conexão sub-Riemanniana) e funções das equações de estrutura (que abaixo aparecem com o sinal acima) e as formas e funções anteriores:

$$
\begin{array}{ll}
\widetilde{\theta}^{1}=\sqrt{c} \theta^{1} & \widetilde{K}=\frac{1}{c} K \\
\tilde{\theta}^{2}=\sqrt{c} \theta^{2} & \widetilde{W}_{i}=\frac{1}{c^{3 / 2}} W_{i}, \quad i=1,2, \\
\widetilde{\theta}^{3}=c \theta^{3} & \widetilde{a}=\frac{1}{c} a \\
\widetilde{\omega}=\omega & \widetilde{b}=\frac{1}{c} b
\end{array}
$$

Portanto, se denotarmos por $S_{r}^{3}$ e $Q_{r}^{3}(r>0)$ a esfera e a quádrica dos exemplos (2) e (3), respectivamente, com a métrica multiplicada por $r^{2}$, podemos resumir os resultado obtidos na seguinte tabela: 


\begin{tabular}{|c|c|c|c|}
\cline { 2 - 4 } \multicolumn{1}{c|}{} & $K$ & $W$ & $\tau$ \\
\hline$H^{3}$ & 0 & 0 & 0 \\
\hline$S_{r}^{3}$ & $\frac{4}{r^{2}}$ & 0 & 0 \\
\hline$Q_{r}^{3}$ & $-\frac{4}{r^{2}}$ & 0 & 0 \\
\hline
\end{tabular}

A justificativa para a notação utilizada se dá pelo fato de $S_{r}^{3}$ e $Q_{r}^{3}$ serem isométricas à esfera e à quádrica de raios $r$, com a métrica induzida pela métrica euclidiana do $\mathbb{R}^{4}$. 


\section{Capítulo 3}

\section{Derivada covariante}

Na primeira seção deste capítulo, introduz-se a derivada covariante associada a uma forma de conexão e a versão do Teorema 2.1 em termos da derivada covariante. A seção 3.2 aparece aqui somente a título de complementação do trabalho, sendo dispensável sua leitura para a compreensão do restante do mesmo. Nela é fornecida a relação entre a derivada covariante e transporte paralelo definido na seção 2.2 .

\subsection{Derivada covariante sub-Riemanniana}

Mostraremos a seguir a equivalência entre fornecer uma forma de conexão em $B(M)$ e dar uma derivada covariante $\nabla$, em $M$, com certas propriedades. Essa equivalência nos fornecerá de imediato uma outra versão do Teorema 2.1 em termos de $\nabla$ (ver [6]).

Sejam $M$ uma variedade sub-Riemanniana de contato de dimensão 3 e $\omega$ uma forma de conexão em $B(M)$. Associaremos então a $\omega$ uma derivada covariante $\nabla$, definida da seguinte forma: se $e: U \rightarrow S(U)$ é uma seção em $U$, utilizando as identificações

$$
\left\{\begin{array}{l}
e_{1}=e \\
e_{2}=i e \\
e_{3}=\xi
\end{array}\right.
$$


se $X$ é campo definido em $U$, tal que $X=\sum_{i=1}^{3} X^{i} e_{i}$, definimos

$$
\nabla X=\sum_{i=1}^{3}\left(\mathrm{~d} X^{i} \otimes e_{i}+X^{i} \nabla e_{i}\right)
$$

onde

$$
\begin{aligned}
\nabla e_{1} & =e^{*} \omega \otimes e_{2} \\
\nabla e_{2} & =-e^{*} \omega \otimes e_{1} \\
\nabla \xi & =0
\end{aligned}
$$

Precisamos mostrar então que (3.1) define bem $\nabla$, mas para tal, basta que mostremos que as derivadas em (3.2) estão bem definidas, ou seja, se $\tilde{e}: U \rightarrow S(U)$ for uma outra seção, que

$$
\begin{aligned}
& \nabla \widetilde{e}_{1}=\tilde{e}^{*} \omega \otimes \widetilde{e}_{2} \\
& \nabla \tilde{e}_{2}=\tilde{e}^{*} \omega \otimes \tilde{e}_{1}
\end{aligned}
$$

Sabemos já que $\tilde{e}=R_{\alpha} . e$, para alguma função diferenciável $\alpha: U \rightarrow \mathbb{R}$. Calculemos então $\nabla \widetilde{e}_{i}:$

Note que, se $X \in T_{m} M$,

$$
\begin{aligned}
\left(\widetilde{e}^{*} \omega\right)_{m}(X) & =\omega_{\widetilde{e}(m)}(\mathrm{d} \widetilde{e}(X))=\omega_{R_{\alpha(m)} e_{(m)}}\left(\mathrm{d} R_{\alpha(m)} \circ \mathrm{d} e(X)+\mathrm{d} \alpha(X) V_{R_{\alpha(m)} e_{(m)}}\right) \\
& =\left(R_{\alpha(m)}^{*} \omega\right)(\mathrm{d} e(X))+\mathrm{d} \alpha(X) \\
& =\omega_{e(m)}(\mathrm{d} e(X))+\mathrm{d} \alpha(X) \\
& =e^{*} \omega_{m}(X)+\mathrm{d} \alpha(X) .
\end{aligned}
$$

Observe então que

$$
\begin{aligned}
\nabla_{X} \tilde{e}_{1} & =\nabla_{X}\left(\cos \alpha \cdot e_{1}+\operatorname{sen} \alpha \cdot e_{2}\right) \\
& =X(\cos \alpha) e_{1}+\cos \alpha \cdot \nabla_{X} e_{1}+X(\operatorname{sen} \alpha) e_{2}+\operatorname{sen} \alpha \nabla_{X} e_{2} \\
& =-\operatorname{sen} \alpha \cdot \operatorname{d} \alpha(X) e_{1}+\cos \alpha \cdot e^{*} \omega(X) e_{2}+\cos \alpha \cdot \operatorname{d} \alpha(X) e_{2}-\operatorname{sen} \alpha \cdot e^{*} \omega(X) e_{1} \\
& =\left(e^{*} \omega(X)+\mathrm{d} \alpha(X)\right)\left(-\operatorname{sen} \alpha \cdot e_{1}+\cos \alpha \cdot e_{2}\right) \\
& =\widetilde{e}^{*} \omega(X) \cdot \tilde{e}_{2}
\end{aligned}
$$




$$
\begin{aligned}
\nabla_{X} \widetilde{e}_{2} & =\nabla_{X}\left(-\operatorname{sen} \alpha \cdot e_{1}+\cos \alpha \cdot e_{2}\right) \\
& =X(-\operatorname{sen} \alpha) e_{1}-\operatorname{sen} \alpha \cdot \nabla_{X} e_{1}+X(\cos \alpha) e_{2}+\cos \alpha \nabla_{X} e_{2} \\
& =-\cos \alpha \cdot \mathrm{d} \alpha(X) e_{1}-\operatorname{sen} \alpha \cdot e^{*} \omega(X) e_{2}-\operatorname{sen} \alpha \cdot \mathrm{d} \alpha(X) e_{2}-\cos \alpha \cdot e^{*} \omega(X) \cdot e_{1} \\
& =-\left(e^{*} \omega(X)+\operatorname{d} \alpha(X)\right)\left(\cos \alpha \cdot e_{1}+\operatorname{sen} \alpha \cdot e_{2}\right) \\
& =-\tilde{e}^{*} \omega(X) \cdot e_{1}
\end{aligned}
$$

Portanto, $\nabla$ está bem definida.

Note que é fácil notar a compatibilidade de $\nabla$ com a métrica $\langle$,$\rangle . De fato, basta$ mostrarmos que

$$
\left\langle\nabla_{X} e_{i}, e_{j}\right\rangle+\left\langle e_{i}, \nabla_{X} e_{j}\right\rangle=X\left(\left\langle e_{i}, e_{j}\right\rangle\right)=X\left(\delta_{i j}\right)=0, \quad i \leq j=1,2,3
$$

mas isso é decorrência imediata de (3.1).

Portanto, associamos a uma forma de conexão $\omega$, uma derivada covariante $\nabla$, que possui as seguintes propriedades:

P1) $\nabla \xi=0$

P2) $\nabla: \mathcal{D} \rightarrow \mathcal{D}$

P3) $\nabla$ é compatível com $\langle$,$\rangle .$

Procederemos agora de forma inversa: dada uma derivada covariante $\nabla$ que satisfaz P1), P2) e P3), associaremos a ela uma forma de conexão $\omega$ da seguinte maneira: suponha $v \in \pi^{-1}(m)$ e seja $c: U \rightarrow S(U), x \in U$, uma seção diferenciável tal que $c(m)=v$. Note primeiro que

$$
T_{v} B(M)=\mathrm{d} c\left(T_{m} M\right) \oplus\left[V_{v}\right]
$$

de fato, é evidente que $c$ é imersão, isto é, $\mathrm{d} c\left(T_{m} M\right)$ tem dimensão 3 e que se $X \in$ $\mathrm{d} c\left(T_{m} M\right) \cap\left[V_{m}\right]$ então $X=\lambda V_{m}, \lambda \in \mathbb{R} \mathrm{e} X=\mathrm{d} c_{m}(Y)$ para algum $Y \in T_{m} M$. Então, a primeira igualdade implica que $\mathrm{d} \pi(X)=0$, mas então $0=\mathrm{d} \pi(X)=\mathrm{d} \pi \circ \mathrm{d} c(Y)=$ 
$\mathrm{d}(\pi \circ c)(Y)=\mathrm{d}\left(I_{d}\right)(Y)=I_{d}(Y)=Y$ e portanto $X=\mathrm{d} c(Y)=\mathrm{d} c(0)=0$. Seja então $X \in T_{v} B(M)$. Então

$$
X=Y+\lambda V_{m} \text { onde } Y \in \mathrm{d} c\left(T_{m} M\right) \text { e } \lambda \in \mathbb{R} .
$$

Definimos então

$$
\omega(X)=\left\langle\left(\nabla_{\mathrm{d} \pi(X)} c\right), i v\right\rangle+\lambda .
$$

Observe primeiro que $\left(\nabla_{\mathrm{d} \pi(X)} c\right)_{m}$ faz sentido, pois se $X$ e $Y$ são campos, é fácil ver que $\left(\nabla_{Y} X\right)_{m}$ só depende de $Y_{(m)}$ e do valor do campo $X$ ao longo de uma curva integral ao campo $Y$ passando por $m$ (ver [3], p.50). Precisamos mostrar agora que $\omega$ está bem definida, isto é, que o valor de $\omega(X)$ independe da seção escolhida. Seja então $\tilde{c}=R_{\alpha} \cdot c$ uma outra seção tal que $\alpha(m)=0$, i.e., $\tilde{c}(m)=c(m)=v$, onde $\alpha: U \rightarrow \mathbb{R}$ é diferenciável (lembre-se que qualquer seção pode ser expressa dessa forma). Note que

$$
\mathrm{d} \widetilde{c}_{(x)}=\mathrm{d}\left(R_{\alpha(x)}\right)_{c(x)} \circ \mathrm{d} c_{x}+\mathrm{d} \alpha_{(x)} \otimes V_{c(x)} .
$$

Mas, se $x=m, \alpha(m)=0 \mathrm{e} \mathrm{d}\left(R_{0}\right)_{c(m)}=d(I d)_{v}=I d_{v}$. Então,

$$
\mathrm{d} \tilde{c}_{(m)}=\mathrm{d} c_{m}+\mathrm{d} \alpha_{(m)} \otimes V_{v}
$$

Seja $X \in T_{v} B(M)$, suponha $X=Y+\lambda V$, onde $Y \in \mathrm{d} c\left(T_{m} M\right)$, então $Y=\mathrm{d} c(\tilde{Y})$, para algum $\tilde{Y} \in T_{m} M$, temos então que

$$
\mathrm{d} \widetilde{c}(\tilde{Y})=\mathrm{d} c(\tilde{Y})+\mathrm{d} \alpha(\tilde{Y}) V=Y+\mathrm{d} \alpha(\tilde{Y}) V .
$$

Note que $\mathrm{d} \pi(X)=\mathrm{d} \pi(Y)=\mathrm{d} \pi \circ \mathrm{d} c(\tilde{Y})=\mathrm{d}(\pi \circ c)(\tilde{Y})=\tilde{Y}$. Então, teremos que

$$
X=Y+\mathrm{d} \alpha(\mathrm{d} \pi(X)) V+(\lambda-\mathrm{d} \alpha(\mathrm{d} \pi(X))) V
$$

onde $Y+\mathrm{d} \alpha(\mathrm{d} \pi(X)) V \in \mathrm{d} \widetilde{c}\left(T_{m} M\right)$. Nosso objetivo então é mostrar que

$$
\omega(X)=\left\langle\nabla_{\mathrm{d} \pi(X)} \widetilde{c}, i v\right\rangle+(\lambda-\mathrm{d} \alpha(\mathrm{d} \pi(X))) .
$$

Para isso, note que

$$
\begin{aligned}
\nabla_{\mathrm{d} \pi(X) \tilde{c}} & =\nabla_{\mathrm{d} \pi(X)}(\cos \alpha \cdot c+\operatorname{sen} \alpha \cdot i c) \\
& =\left[\mathrm{d} \pi(X)(\cos \alpha) c+\cos \alpha \nabla_{\mathrm{d} \pi(X)} c+\mathrm{d} \pi(X)(\operatorname{sen} \alpha) i c+\operatorname{sen} \alpha \cdot \nabla_{\mathrm{d} \pi(X)} i c\right]_{m} \\
& =\left[-\operatorname{sen} \alpha \cdot \mathrm{d} \alpha(\mathrm{d} \pi(X)) c+\cos \alpha \cdot \mathrm{d} \alpha(\mathrm{d} \pi(X)) i c+\cos \alpha \nabla_{\mathrm{d} \pi(X)} c+\operatorname{sen} \alpha \nabla_{\mathrm{d} \pi(X)} i c\right]_{m}
\end{aligned} .
$$


Mas $\alpha(m)=0$ e $c(m)=v$, então,

$$
\nabla_{\mathrm{d} \pi(X)} \tilde{c}=\mathrm{d} \alpha(\mathrm{d} \pi(X)) \cdot i v+\nabla_{\mathrm{d} \pi(X)} c .
$$

Portanto, teremos que

$$
\begin{aligned}
\omega(X) & =\left\langle\nabla_{\mathrm{d} \pi(X)} c, i v\right\rangle+\lambda \\
& =\left\langle\nabla_{\mathrm{d} \pi(X)} \tilde{c}-\mathrm{d} \alpha(\mathrm{d} \pi(X)) i v, i v\right\rangle+\lambda \\
& =\left\langle\nabla_{\mathrm{d} \pi(X)} \tilde{c}, i v\right\rangle+\lambda-\mathrm{d} \alpha(\mathrm{d} \pi(X))
\end{aligned}
$$

como queríamos demonstrar.

Precisamos mostrar agora que $\omega$ é forma de conexão, i.e., que as duas condições abaixo são satisfeitas:

i) $\omega(V)=1$;

ii) $R_{\alpha}^{*} \omega=\omega, \forall \alpha \in[0,2 \pi[$.

A condição i) é imediata da definição de $\omega$. Verifiquemos então-ii):

Sejam então $v \in B(M), X \in T_{v} B(M)$ e $\alpha \in\left[0,2 \pi\left[\right.\right.$. Queremos mostrar que $\left(R_{\alpha}^{*} \omega\right)_{v}(X)=$ $\omega_{v}(X)$ ou, melhor dizendo, que $\omega_{R_{\alpha v}}\left(\mathrm{~d} R_{\alpha}(X)\right)=\omega_{v}(X)$.

Antes note que se $c: U \rightarrow S(U)$ é uma seção que passa por $v$, i.e., $c(m)=v$, então $\tilde{c}=R_{\alpha} c$ é seção que passa por $R_{\alpha} v$. Além disso, se $X$ se decompõe

$$
X=Y+\lambda V
$$

onde $Y \in \mathrm{d} c\left(T_{m} M\right)$, teremos que

$$
\mathrm{d} R_{\alpha}(X)=\mathrm{d} R_{\alpha}(Y)+\lambda \mathrm{d} R_{\alpha}(V)
$$

Mas é fácil ver que $\mathrm{d} R_{\alpha}(Y) \in \mathrm{d} \tilde{c}\left(T_{m} M\right)$, pois, se $Y \in \mathrm{d} c\left(T_{m} M\right)$, então $\mathrm{d} R_{\alpha}(Y) \in$ $\mathrm{d} R_{\alpha}\left(\mathrm{d} c\left(T_{m} M\right)\right)=\mathrm{d} \widetilde{c}\left(T_{m} M\right)$, e que $\mathrm{d} R_{\alpha}\left(V_{v}\right)=V_{R_{\alpha} v}$, pois

$$
\mathrm{d} R_{\alpha}\left(V_{v}\right)=\mathrm{d} R_{\alpha}\left(\left.\frac{\mathrm{d}}{\mathrm{d} t} R_{t} \cdot v\right|_{t=0}\right)=\left.\frac{\mathrm{d}}{\mathrm{d} t}\left(R_{\alpha} \cdot R_{t} \cdot v\right)\right|_{t=0}=\left.\frac{\mathrm{d}}{\mathrm{d} t}\left(R_{t} \cdot R_{\alpha} \cdot v\right)\right|_{t=0}=V_{R_{\alpha} v} .
$$


Temos então que $\mathrm{d} R_{\alpha}(X)$ se decompõe

$$
\mathrm{d} R_{\alpha}(X)=\mathrm{d} R_{\alpha}(Y)+\lambda V
$$

onde $\mathrm{d} R_{\alpha}(Y) \in \mathrm{d} \widetilde{c}\left(T_{m} M\right)$. Então,

$$
\begin{aligned}
\omega_{R_{\alpha} v}\left(\mathrm{~d} R_{\alpha}(X)\right)= & \left\langle\nabla_{\mathrm{d} \pi \circ \mathrm{d} R_{\alpha}(X)} \tilde{c}, i R_{\alpha} v\right\rangle+\lambda \\
= & \left\langle\cos \alpha \nabla_{\mathrm{d} \pi(X)} c+\operatorname{sen} \alpha \nabla_{\mathrm{d} \pi(X)} i c,-\operatorname{sen} \alpha \cdot v+\cos \alpha \cdot i v\right\rangle+\lambda \\
= & \cos ^{2} \alpha\left\langle\nabla_{\mathrm{d} \pi(X)} c, i v\right\rangle-\operatorname{sen}^{2} \alpha\left\langle\nabla_{\mathrm{d} \pi(X)} i c, v\right\rangle-\operatorname{sen} \alpha \cos \alpha\left\langle\nabla_{\mathrm{d} \pi(X)} c, v\right\rangle \\
& +\operatorname{sen} \alpha \cos \alpha\left\langle\nabla_{\mathrm{d} \pi(X)} i c, i v\right\rangle+\lambda .
\end{aligned}
$$

Mas, da compatibilidde de $\nabla$ em relação à métrica, é fácil ver que $\left\langle\nabla_{\mathrm{d} \pi(X)} i c, v\right\rangle=$ $-\left\langle\nabla_{\mathrm{d} \pi(X)} c, i v\right\rangle$ e que $\left\langle\nabla_{\mathrm{d} \pi(X)} c, v\right\rangle=\left\langle\nabla_{\mathrm{d} \pi(X)} i c, i v\right\rangle=0$, pois basta notar que

$$
0=\mathrm{d} \pi(X)(0)=\mathrm{d} \pi(X)(\langle c, i c\rangle)=\left\langle\nabla_{\mathrm{d} \pi(X)} c, i v\right\rangle+\left\langle v, \nabla_{\mathrm{d} \pi(X)^{i}} i\right\rangle
$$

e fazer o análogo para $\mathrm{d} \pi(X)(\langle c, c\rangle)$ e $\mathrm{d} \pi(X)(\langle i c, i c\rangle)$. Observe então que

$$
\begin{aligned}
\omega_{R_{\alpha} v}\left(\mathrm{~d} R_{\alpha}(X)\right) & =\left(\cos ^{2} \alpha+\operatorname{sen}^{2} \alpha\right)\left\langle\nabla_{\mathrm{d} \pi(X)} c, i v\right\rangle+\lambda \\
& =\left\langle\nabla_{\mathrm{d} \pi(X)} c, i v\right\rangle+\lambda=\omega_{v}(X)
\end{aligned}
$$

e a condição ii) está mostrada.

Construímos então uma função $\varphi$, que a cada derivada covariante $\nabla$, com propriedades P1, P2 e P3, corresponde uma forma de conexão $\varphi(\nabla)$. Antes já havíamos construído uma outra função $\phi$, que a cada forma de conexão $\omega$, correspondia uma derivada covariante $\phi(\omega)$ com propriedades P1, P2, P3. Mostraremos agora que as duas funções construídas são inversas: seja então $\omega$ forma de conexão e $\nabla=\phi(\omega)$. Queremos mostrar que $\varphi(\nabla)=\omega$, que implicaria que $\varphi \circ \phi=I_{d}$. Sejam então $\tilde{\omega}=\varphi(\nabla), v \in B(M), X \in T_{v} B(M)$ e $c$ seção que passa por $v$. Teremos então que

$$
\widetilde{\omega}(X)=\left\langle\nabla_{\mathrm{d} \pi(X)} c, i v\right\rangle+\lambda, \text { onde } X=Y+\lambda V, Y \in \mathrm{d} c\left(T_{m} M\right) .
$$

Mas como $\nabla=\phi(\omega)$, temos que $\nabla_{\mathrm{d} \pi(X)} c=\left(c^{*} \omega\right)(\mathrm{d} \pi(X)) . i v$ e portanto

$$
\tilde{\omega}(X)=c^{*} \omega(\mathrm{d} \pi(X))+\lambda
$$




$$
\begin{aligned}
& =\omega(\mathrm{d} c 0 \mathrm{~d} \pi(X))+\lambda .1 \\
& =\omega(Y)+\lambda \omega(V) \\
& =\omega(Y+\lambda V) \\
& =\omega(X)
\end{aligned}
$$

Portanto, $\varphi \circ \phi(\omega)=\omega$. Suponha agora $\nabla$ derivada covariante com as propriedades P1, P2 e P3, $\varphi(\nabla)=\omega$ e $\widetilde{\nabla}=\phi(\omega)$. Sejam $c$ uma seção e $X$ campo. Então teremos que

$$
\begin{aligned}
\widetilde{\nabla}_{X} c & =c^{*} \omega(X) \cdot i c \\
\widetilde{\nabla}_{X} i c & =-c^{*} \omega(X) \cdot c \\
\widetilde{\nabla} \xi & =0
\end{aligned}
$$

Agora,

$$
c^{*} \omega(X)=\omega(\mathrm{d} c(X))=\left\langle\nabla_{\mathrm{d} \pi \operatorname{od} c(X)} c, i c\right\rangle=\left\langle\nabla_{X} c, i c\right\rangle .
$$

Além disso, sabemos que $\nabla: \mathcal{D} \rightarrow \mathcal{D}$ e portanto que $\nabla_{X} c$ não possui componente na direção $\xi$ e segue da compatibilidade de $\nabla$ com a métrica que $\nabla_{X} c$ não possui componente na direção $c$. Resumindo, sabemos que

$$
\nabla_{X} c=\left\langle\nabla_{X} c, i c\right\rangle . i c
$$

e portanto

$$
\widetilde{\nabla}_{X} c=c^{*} \omega(X) \cdot i c=\left\langle\nabla_{X} c, i c\right\rangle \cdot i c=\nabla_{X} c .
$$

De forma análoga, mostramos que

$$
\widetilde{\nabla}_{X} i c=\nabla_{X} i c
$$

e, como $\nabla$ possui a propriedade $\mathrm{P} 1, \nabla \xi=0$, temos que

$$
\widetilde{\nabla}_{X} \xi=0=\nabla_{X} \xi
$$

Como $\widetilde{\nabla}$ e $\nabla$ coincidem em um referencial, então $\widetilde{\nabla}=\nabla$ e $\phi \circ \varphi(\nabla)=\nabla$. Portanto, $\phi$ (ou $\varphi$ ) nos dá uma relação biunívoca entre o conjunto das formas de conexão em $B(M)$ e o conjunto das derivadas covariantes com propriedades P1, P2 e P3. Verifiquemos agora como ficam as equações de estrutura em termos da derivada covariante. 
Seja então $\omega$ uma forma de conexão em $B(M)$ com as seguintes equações de estrutura

$$
\begin{aligned}
\mathrm{d} \omega & =-K \theta^{1} \wedge \theta^{2}+W \wedge \theta^{3} \\
\mathrm{~d} \theta^{1} & =\omega \wedge \theta^{2}+\tau^{1} \wedge \theta^{3}+f \theta^{1} \wedge \theta^{2} \\
\mathrm{~d} \theta^{2} & =-\omega \wedge \theta^{1}+\tau^{2} \wedge \theta^{3}+g \theta^{1} \wedge \theta^{2} \\
\mathrm{~d} \theta^{3} & =2 \theta^{1} \wedge \theta^{2},
\end{aligned}
$$

onde $W=W_{1} \theta^{1}+W_{2} \theta^{2}, \tau^{1}=a \theta^{1}+b \theta^{2}$ e $\tau^{2}=c \theta^{1}-a \theta^{2}$. Seja também $\nabla=\phi(\omega)$ e $T$ a torção de $\nabla$, isto é,

$$
T(X, Y)=\nabla_{X} Y-\nabla_{Y} X-[X, Y]
$$

fazendo o "pull-back" por uma seção (local) c, as equações de estrutura se escreverão de forma análoga, onde $\left(\theta^{1}, \theta^{2}, \theta^{3}\right)$ passam a ser a base dual a $(c, i c, \xi)$ que denotaremos doravante por $\left(e_{1}, e_{2}, e_{3}\right)$. Lembrando que $\theta^{i}\left(e_{j}, e_{k}\right)=-\mathrm{d} \theta^{i}\left(e_{j}, e_{k}\right)$, é simples calcular que

$$
\begin{aligned}
& T\left(e_{1}, e_{2}\right)=f e_{1}+g e_{2}+2 e_{3} \\
& T\left(e_{1}, e_{3}\right)=a e_{1}+c e_{2} \\
& T\left(e_{2}, e_{3}\right)=b e_{1}-a e_{2}
\end{aligned}
$$

Portanto,

$T=\left(f \theta^{1} \wedge \theta^{2}+a \theta^{1} \wedge \theta^{3}+b \theta^{2} \wedge \theta^{3}\right) \otimes e_{1}+\left(g \theta^{1} \wedge \theta^{2}+c \theta^{1} \wedge \theta^{3}-a \theta^{2} \wedge \theta^{3}\right) \otimes e_{2}+2 \theta^{1} \wedge \theta^{2} \otimes e_{3}$.

Segue então da relação biunívoca obtida, da expressão da torsão acima e do teorema de existência e unicidade da forma de conexão sub-Riemanniana, o seguinte teorema, cuja generalização para dimensões superiores pode ser encontrada em [6]

Teorema 3.1 Dada uma variedade sub-Riemanniana de contato $(M, \mathcal{D},\langle\rangle$,$) , com for-$ ma de contato $\theta$, existe um único tensor $\tau$ simétrico e uma única derivada covariante tais que

i) $\nabla: \mathcal{D} \rightarrow \mathcal{D}$

ii) $\nabla \xi=0$; 
iii) $\nabla$ é compativel com $\langle$,$\rangle ;$

iv) $\left.T\right|_{\mathcal{D}}=\left.\mathrm{d} \theta\right|_{\mathcal{D}} \otimes \xi$,

$T(X, \xi)=\tau(X)$,

onde $T$ é o tensor torção de $\nabla$.

Repare que podemos recuperar facilmente as funções das equações de estrutura em função da torção e curvatura. Se $\tau=\left(a \theta^{1}+b \theta^{2}\right) \otimes e_{1}+\left(b \theta^{1}-a \theta^{2}\right) \otimes e_{2}$,

$$
\begin{aligned}
a & =\left\langle\tau\left(e_{1}\right), e_{1}\right\rangle=\left\langle T\left(e_{1}, \xi\right), e_{1}\right\rangle \\
b & =\left\langle\tau\left(e_{2}\right), e_{1}\right\rangle=\left\langle T\left(e_{2}, \xi\right), e_{1}\right\rangle \\
W_{1} & =\left\langle R\left(\xi, e_{1}\right) e_{1}, e_{2}\right\rangle=-\left\langle R\left(\xi, e_{1}\right) e_{2}, e_{1}\right\rangle \\
W_{2} & =\left\langle R\left(\xi, e_{2}\right) e_{1}, e_{2}\right\rangle
\end{aligned}
$$

\subsection{Relação entre derivada covariante e transporte paralelo}

Daremos através da próxima proposição uma forma de se obter a derivada covariante (associada a uma conexão) a partir do transporte paralelo. Essa maneira alternativa de definir a derivada covariante nos permite uma visão mais geométrica da mesma.

Proposição 3.1 Seja $M$ uma variedade sub-Riemanniana de contato de dimensão 3, $\omega$ uma forma de conexão e $\nabla$ a derivada covariante associada. Se $X$ e $Y$ são campos diferenciáveis definidos em uma vizinhança $U$ de $m \in M$, então

$$
\left(\nabla_{Y} X\right)_{m}=\lim _{t \rightarrow 0} \frac{X_{\gamma(t)}-T P_{\gamma(t)}\left(X_{m}\right)}{t}
$$

onde $\gamma:(-\varepsilon, \varepsilon) \rightarrow M$ é curva diferenciável, com $\gamma(0)=m$ e $\dot{\gamma}(0)=Y_{m}$, regular, se $Y_{m} \neq 0$ ou constante se $Y_{m}=0$. 
Prova: No caso $Y_{m}=0$, é imediato da definição de $\nabla$ e do transporte paralelo, que ambos os membros da equação se anulam. Suponhamos então $Y_{m} \neq 0$. Seja $e: U \rightarrow S(U)$ uma seção diferenciável (para isso, podemos supor $U$ suficientemente pequeno). Mostraremos primeiro que (3.3) vale para $X=e_{i}, i=1,2,3$, onde $e_{1}=e, e_{2}=i e$ e $e_{3}=\xi$.

No caso em que $X=e_{3}=\xi$, por definição, $\nabla \xi=0$ e como $\xi$ é paralelo, isto é, $T P_{\gamma(t)}\left(\xi_{m}\right)=\xi_{\gamma(t)}$, é claro que o segundo membro de (3.3) também se anula. No caso em que $X=e_{1}$, segue da observação feita no final da seção 2.2 que

$$
e_{1 \gamma(t)}=R_{\theta(t)} \cdot T P_{\gamma(t)}\left(e_{1_{m}}\right),
$$

onde $\theta:(-\varepsilon, \varepsilon) \rightarrow \mathbb{R}$ é diferenciável, $\theta(0)=0 \mathrm{e}$

$$
\frac{\mathrm{d} \theta}{\mathrm{d} t}(s)=\omega\left(\left.\frac{\mathrm{d}}{\mathrm{d} t}\left(e_{\gamma(t)}\right)\right|_{t=s}\right)=\left(e^{*} \omega\right)(\dot{\gamma}(s)) .
$$

Note então que

$$
\begin{aligned}
\lim _{t \rightarrow 0} \frac{e_{1 \gamma(t)}-T P_{\gamma(t)}\left(e_{1 m}\right)}{t} & =\lim _{t \rightarrow 0} \frac{R_{\theta(t)}-1}{t} T P_{\gamma(t)}\left(e_{1 m}\right) \\
& =\left.\frac{\mathrm{d}}{\mathrm{d} t}\left(R_{\theta(t)}\right)\right|_{t=0} \cdot e_{1_{m}} \\
& =\left.\left.\frac{\mathrm{d}}{\mathrm{d} \theta}\left(R_{\theta}\right)\right|_{\theta=0} \cdot \frac{\mathrm{d} \theta}{\mathrm{d} t}\right|_{t=0} \cdot e_{1_{m}} \\
& =i \frac{\mathrm{d} \theta}{\mathrm{d} t}(0) \cdot e_{1_{m}} \\
& =\left(e^{*} \omega\right)(\dot{\gamma}(0)) i e_{1_{m}} \\
& =\left(e^{*} \omega\right)\left(Y_{m}\right) e_{2_{m}} \\
& =\left(\nabla_{Y} e_{1}\right)_{m}
\end{aligned}
$$

Analogamente, mostramos (3.3) para $X=e_{2}$. Note agora que, se $X=\sum X^{i} e_{i}$, do fato de o transporte paralelo ser isometria entre espaços vetoriais, temos que

$$
\begin{aligned}
\lim _{t \rightarrow 0} \frac{X_{\gamma(t)}-T P_{\gamma(t)}\left(X_{m}\right)}{t} & =\lim _{t \rightarrow 0} \sum_{i=1}^{3}\left[\frac{\left(X^{i} e_{i}\right)_{\gamma(t)}-X_{m}^{i} T P_{\gamma(t)}\left(e_{i_{m}}\right)}{t}\right] \\
& =\sum_{i=1}^{3} \lim _{t \rightarrow 0}\left[\frac{X^{i}(\gamma(t))-X_{m}^{i}}{t} \cdot e_{i \gamma(t)}+X_{m}^{i} \frac{e_{i \gamma(t)}-T P_{\gamma(t)}\left(e_{i_{m}}\right)}{t}\right] \\
& =\sum_{i=1}^{3}\left[\mathrm{~d} X^{i}\left(Y_{m}\right) e_{i_{m}}+X_{m}^{i}\left(\nabla_{Y} e_{i}\right)_{m}\right] \\
& =\left(\nabla_{Y} X\right)_{m}
\end{aligned}
$$


É bem fácil notar agora, a partir de (3.3), que $\left(\nabla_{Y} X\right)$ só depende do valor de $Y$ em $m$ e do comportamento de $X$ ao longo de $\gamma$. Faz sentido então, dado $v \in T_{m} M$, falar em $\nabla_{v} X$. A proposição que segue, dá-nos uma caracterização do transporte paralelo em termos de $\nabla$.

Proposição 3.2 Seja $U$ uma vizinhança de $m \in M$ e $\gamma:(-\varepsilon, \varepsilon) \rightarrow U$, uma curva diferenciável, regular, com $\gamma(0)=m$, e X campo diferenciável, definido em $U$. Então vale

$$
\nabla_{\dot{\gamma}(t)} X=0, \quad \forall t \in(-\varepsilon, \varepsilon) \Leftrightarrow X_{\gamma(t)}=T P_{\gamma(t)}\left(X_{m}\right)
$$

Prova: Seja $e: U \rightarrow S(U)$, uma seção diferenciável (novamente podemos supor $U$ suficientemente pequeno, para tal). Escrevamos $X=f e+g \xi$. Temos então, por definição, que

$$
T P_{\gamma(t)}\left(X_{\gamma(s)}\right)=f(\gamma(s)) T P_{\gamma(t)}\left(e_{\gamma(s)}\right)+g(\gamma(s)) \xi_{\gamma(t)},
$$

mas sabemos que $e_{\gamma(s)}=R_{\alpha(s)} T P_{\gamma(s)}\left(e_{m}\right)$, onde $\alpha:(-\varepsilon, \varepsilon) \rightarrow \mathbb{R}$ é diferenciável e $\alpha(0)=0$. De (2.4), temos então que

$$
T P_{\gamma(t)}\left(X_{\gamma(s)}\right)=f(\gamma(s)) R_{\alpha(s)} T P_{\gamma(t)}\left(e_{m}\right)+g(\gamma(s)) \xi_{\gamma(t)} .
$$

Observe que utilizamos acima o fato, fácil de ser mostrado a partir da unicidade do levantamento, de que $T P_{\gamma(t)}\left(T P_{\gamma(s)}\left(e_{m}\right)\right)=T P_{\gamma(t)}\left(e_{m}\right)$. Agora é fácil ver através de uma translação em $t$, que podemos escrever

$$
\nabla_{\dot{\gamma}(s)} X=\lim _{t \rightarrow s} \frac{X_{\gamma(t)}-T P_{\gamma(t)}\left(X_{\gamma(s)}\right)}{t-s}
$$

então teremos que

$$
\begin{aligned}
\nabla_{\dot{\gamma}(s)} X & =\lim _{t \rightarrow s}\left[\frac{f(\gamma(t)) R_{\alpha(t)}-f(\gamma(s)) R_{\alpha(s)}}{t-s} T P_{\gamma(t)}\left(e_{m}\right)+\frac{g(\gamma(t))-g(\gamma(s))}{t-s} \xi_{\gamma(t)}\right] \\
& =\frac{\mathrm{d}}{\mathrm{d} t}\left[f(\gamma(t)) R_{\alpha(t)}\right]_{t=s} T P_{\gamma(s)}\left(e_{m}\right)+\frac{\mathrm{d}}{\mathrm{d} t}[g(\gamma(t))]_{t=s} \xi_{\gamma(s)}
\end{aligned}
$$

e é claro então que

$$
\nabla_{\dot{\gamma}(s)} X=0, \forall s \in(-\varepsilon, \varepsilon) \Leftrightarrow\left\{\begin{array}{l}
f(\gamma(s)) R_{\alpha(s)}=f(\gamma(0)) R_{\alpha(0)}=f(m) \\
g(\gamma(s))=g(\gamma(0))=g(m)
\end{array}, \forall s \in(-\varepsilon, \varepsilon) .\right.
$$


Mas como

$$
X_{\gamma(s)}=f(\gamma(s)) R_{\alpha(s)} T P_{\gamma(s)}\left(e_{m}\right)+g(\gamma(s)) T P_{\gamma(s)}\left(\xi_{m}\right)
$$

temos que

$$
\begin{aligned}
\nabla_{\dot{\gamma}(s)} X=0, \forall s \in(-\varepsilon, \varepsilon) \Leftrightarrow X_{\gamma(s)} & =f(m) T P_{\gamma(s)}\left(e_{m}\right)+g(m) T P_{\gamma(s)}\left(\xi_{m}\right) \\
& =T P_{\gamma(s)}\left(f(m) e_{m}+g(m) \xi_{m}\right) \\
& =T P_{\gamma(s)}\left(X_{m}\right), \quad \forall s \in(-\varepsilon, \varepsilon) .
\end{aligned}
$$




\section{Capítulo 4}

\section{Classificação das variedades homogêneas}

Na primeira seção desse último capítulo, introduzem-se os conceitos de isometria entre variedades sub-Riemannianas e de variedades homogêneas. Na seção 4.2 são calculados os grupos de isometrias "positivas" de $H^{3}, S^{3}$ e $Q^{3}$, cujo conhecimento é utilizado na seção 4.3.

É precisamente na seção 4.3 onde aparecem os resultados principais do trabalho: os teoremas de classificação 4.2 e 4.3 .

Por fim, na última seção apresentamos a classificação das variedades homogêneas em termos de seus invariantes $K, \lambda \geq 0, W_{1} \geq 0, W_{2}$.

\subsection{Isometria sub-Riemanniana, variedade homogê- nea}

Definição 4.1 Seja $f: M \rightarrow N$ uma aplicação diferenciável entre duas variedades subRiemannianas de contato $(M, \mathcal{D},\langle\rangle$,$) e \left(N, \mathcal{D}^{\prime},\langle\rangle,\right)$ que satisfaça

i) $\mathrm{d} f_{m}\left(\mathcal{D}_{m}\right)=\mathcal{D}_{m}^{\prime}$; 
ii) $\left\langle\mathrm{d} f_{m}(X), \mathrm{d} f_{m}(Y)\right\rangle=\langle X, Y\rangle, \quad \forall X, Y \in \mathcal{D}_{m}$,

qualquer que seja $m \in M$, então $f$ é dita uma isometria local (sub-Riemanniana) entre $M$ e N. Se além disso, $f$ for bijetora, diremos apenas que $f$ é uma isometria subRiemanniana entre $M$ e $N$.

Note que, se $f: M \rightarrow N$ é isometria local e $(\theta, \xi)$ e $(\tilde{\theta}, \tilde{\xi})$ são as formas de contato e os campos fundamentais associados de $M$ e $N$, respectivamente, então, como $f$ preserva a distribuição e o elemento de área (a menos de mudança de sinal), é imediato que $f^{*} \tilde{\theta}= \pm \theta$ e que $f_{*} \xi= \pm \tilde{\xi}$, onde o sinal é determinado pelo fato de $\mathrm{d} f$ preservar ou não a orientação na distribuição. É imediato então notar que toda isometria local é difeomorfismo local.

Usaremos a seguinte notação para os grupos de isometria:

$$
\begin{aligned}
\operatorname{Isom}(M) & =\{f: M \rightarrow M \mid f \text { é isometria }\} \\
\text { Isom }^{+}(M) & =\{f \in \operatorname{Isom}(M) \mid f \text { preserva orientação em } \mathcal{D}\} .
\end{aligned}
$$

Definição 4.2 Uma variedade sub-Riemanniana de contato $M$ é dita homogênea se o grupo $\operatorname{Isom}(M)$ opera transitivamente em $M$.

\subsection{Grupos de isometrias de $H^{3}, S^{3}$ e $Q^{3}$}

A seguir calcularemos o grupo das isometrias "positivas" dos três exemplos discutidos anteriormente e concluiremos que tais grupos atuam transitivamente nos fibrados de círculos.

(1) $H^{3}$

Recordemos que em $H^{3}$, temos:

$$
\begin{cases}e_{1}=\frac{\partial}{\partial x}-y \frac{\partial}{\partial z}, & \theta=-y \mathrm{~d} x+x \mathrm{~d} y-\mathrm{d} z \\ e_{2}=\frac{\partial}{\partial y}+x \frac{\partial}{\partial z}, & g=\mathrm{d} x^{2}+\mathrm{d} y^{2} \\ \xi=-\frac{\partial}{\partial z}, & \end{cases}
$$


onde $\left\{e_{1}, e_{2}\right\}$ é base ortonormal da distribuição de contato $\mathcal{D}, \theta$ é a forma de contato associada, $g$ é a métrica em $\mathcal{D}$ e $\xi$ o campo fundamental.

Suponha $F: \mathbb{R}^{3} \rightarrow \mathbb{R}^{3}$ uma isometria (que preserva orientação) e seja $p=(x, y, z) \in$ $\mathbb{R}^{3}$. Calculemos então $\mathrm{d} F_{p}$. Denotemos por $\left(a_{i j}\right)$ a matriz de $\mathrm{d} F_{p}$ em relação à base $\left(\frac{\partial}{\partial x}, \frac{\partial}{\partial y}, \frac{\partial}{\partial z}\right)$. Como $F$ é isometria, segue que $\mathrm{d} F_{p}(\xi)=\xi_{F(p)}$ e portanto $\mathrm{d} F_{p}\left(\frac{\partial}{\partial z}\right)=$ $\frac{\partial}{\partial z}(F(p))$. Logo, necessariamente, $a_{13}=a_{23}=0$ e $a_{33}=1$. Temos então que

$$
\begin{aligned}
& \mathrm{d} F\left(e_{1}\right)=a_{11} \frac{\partial}{\partial x}+a_{21} \frac{\partial}{\partial y}+\left(a_{31}-y\right) \frac{\partial}{\partial z} \\
& \mathrm{~d} F\left(e_{2}\right)=a_{12} \frac{\partial}{\partial x}+a_{22} \frac{\partial}{\partial y}+\left(a_{32}+x\right) \frac{\partial}{\partial z},
\end{aligned}
$$

mas, por $F$ ser isometria, segue que $\left\{\mathrm{d} F_{p}\left(e_{1}\right), \mathrm{d} F_{p}\left(e_{2}\right)\right\}$ é base ortonormal de $\mathcal{D}_{F(p)}$ e, como $g=\mathrm{d} x^{2}+\mathrm{d} y^{2}$, segue que $\left[\begin{array}{ll}a_{11} & a_{21} \\ a_{12} & a_{22}\end{array}\right] \in S O(2)$, isto é, $a_{11}=a_{22}=\cos \alpha(p)$ e $a_{21}=-a_{12}=\operatorname{sen} \alpha(p)$. É fácil notar, no entanto, que $\alpha$ não depende de $p$. De fato, se denotarmos $F=\left(f_{1}, f_{2}, f_{3}\right)$, temos que

$$
\begin{aligned}
& \frac{\partial f_{1}}{\partial x}=a_{11}=\cos \alpha(p)=a_{22}=\frac{\partial f_{2}}{\partial y} \\
& \frac{\partial f_{1}}{\partial y}=a_{12}=-\operatorname{sen} \alpha(p)=-a_{21}=-\frac{\partial f_{2}}{\partial x} .
\end{aligned}
$$

Do teorema de Schwarz, $\frac{\partial^{2} f_{i}}{\partial x \partial y}=\frac{\partial^{2} f_{i}}{\partial y \partial x}, i=1,2$, obtemos as equações

$$
\left\{\begin{array}{l}
-\operatorname{sen} \alpha(p) \frac{\partial \alpha}{\partial y}(p)=-\cos \alpha(p) \frac{\partial \alpha}{\partial x}(p) \\
\cos \alpha(p) \frac{\partial \alpha}{\partial y}(p)=-\operatorname{sen} \alpha(p) \frac{\partial \alpha}{\partial x}(p)
\end{array}\right.
$$

cuja única solução é $\frac{\partial \alpha}{\partial x}(p)=\frac{\partial \alpha}{\partial y}(p)=0$. Temos ainda que

$$
\left\{\begin{array}{l}
\frac{\partial^{2} f_{1}}{\partial z \partial x}(p)=-\operatorname{sen} \alpha(p) \frac{\partial \alpha}{\partial z}(p) \\
\frac{\partial^{2} f_{1}}{\partial z \partial y}(p)=-\cos \alpha(p) \frac{\partial \alpha}{\partial z}(p)
\end{array}\right.
$$

mas já sabemos que $\frac{\partial f_{1}}{\partial z}=a_{13}=0$, portanto segue novamente do teorema de Schwarz que

$$
\left\{\begin{array}{l}
-\operatorname{sen} \alpha(z) \frac{\partial \alpha}{\partial z}(p)=0 \\
-\cos \alpha(z) \frac{\partial \alpha}{\partial z}(p)=0
\end{array}\right.
$$


o que implica que $\frac{\partial \alpha}{\partial z}(p)=0$. Portanto, $\alpha(p)=\alpha$ constante.

Seja $\left(x_{0}, y_{0}, z_{0}\right)=F(0,0,0)$. Observe que já sabemos então que

$$
\left\{\begin{array}{l}
f_{1}(x, y, z)=x \cos \alpha-y \operatorname{sen} \alpha+x_{0} \\
f_{2}(x, y, z)=x \operatorname{sen} \alpha+y \cos \alpha+y_{0}
\end{array}\right.
$$

Mas temos ainda que $\theta_{F(p)}\left(\mathrm{d} F_{p}\left(e_{1}\right)\right)=0=\theta_{F(p)}\left(\mathrm{d} F_{p}\left(e_{2}\right)\right)$. Portanto, já utilizando $(*)$, obtemos

$$
\begin{aligned}
& -\left(x \operatorname{sen} \alpha+y \cos \alpha+y_{0}\right) \cos \alpha+\left(x \cos \alpha-y \operatorname{sen} \alpha+x_{0}\right) \operatorname{sen} \alpha-\left(a_{31}-y\right)=0 \\
& -\left(x \operatorname{sen} \alpha+y \cos \alpha+y_{0}\right)(-\operatorname{sen} \alpha)+\left(x \cos \alpha-y \operatorname{sen} \alpha+x_{0}\right) \cos \alpha-\left(a_{32}+x\right)=0
\end{aligned}
$$

que nos dão

$$
\begin{aligned}
& a_{31}=x_{0} \operatorname{sen} \alpha-y_{0} \cos \alpha \\
& a_{32}=x_{0} \cos \alpha+y_{0} \operatorname{sen} \alpha
\end{aligned}
$$

Portanto, $F$ é dada por

$$
\begin{aligned}
F_{(x, y, z)}= & \left(x \cos \alpha-y \operatorname{sen} \alpha+x_{0}, x \operatorname{sen} \alpha+y \cos \alpha+y_{0},\left(x_{0} \operatorname{sen} \alpha-y_{0} \cos \alpha\right) x\right. \\
& \left.+\left(x_{0} \cos \alpha+y_{0} \operatorname{sen} \alpha\right) y+z+z_{0}\right)
\end{aligned}
$$

para algum $\alpha \in \mathbb{R}$, ou ainda por

$$
F_{(p)}=T_{(p)}+p_{0}
$$

onde $p_{0}=F_{(0)}=\left(x_{0}, y_{0}, z_{0}\right)$ e $T$ é a aplicação linear cuja matriz $[T]$ em relação à base canônica é

$$
[T]=\left[\begin{array}{ccc}
\cos \alpha & -\operatorname{sen} \alpha & 0 \\
\operatorname{sen} \alpha & \cos \alpha & 0 \\
x_{0} \operatorname{sen} \alpha-y_{0} \cos \alpha & x_{0} \cos \alpha+y_{0} \operatorname{sen} \alpha & 1
\end{array}\right]
$$

É evidente agora (e simples de mostrar) que, quaisquer que sejam $\alpha \in \mathbb{R}$ e $\left(x_{0}, y_{0}, z_{0}\right)$, $F$ será uma isometria. Está determinado então $\operatorname{Isom}^{+}\left(H^{3}\right)$. É claro que $\operatorname{Isom}^{+}\left(H^{3}\right)$ atua transitivamente em $H^{3}$, pois dados $p_{1}, p_{2} \in \mathbb{R}^{3}$, se $F_{i}, i=1,2$, são isometrias que levam a origem em $p_{i}$, respectivamente, é claro que $F_{2} \circ F_{1}^{-1}$ é isometria que 
leva $p_{1}$ em $p_{2}$. Note mais: o grupo $\operatorname{Isom}\left(H^{3}\right)$ age transitivamente em $B\left(H^{3}\right)$. Basta observar que o grupo de isotropia da origem

$$
\operatorname{Isom}_{0}\left(H^{3}\right)=\left\{T \in G L(3, \mathbb{R}) \mid[T]=\left[\begin{array}{cc}
A & 0 \\
0 & 1
\end{array}\right], A \in S O(2, \mathbb{R})\right\}
$$

é isomorfo a $S^{1}$ (pois $S O(2, \mathbb{R}) \cong S^{1}$ ) e opera transitivamente em $\pi^{-1}(0)$.

(2) $S^{3}$

É evidente, pela forma como foi construída a esfera, que $U(2) \subset \operatorname{Isom}^{+}\left(S^{3}\right)$. A recíproca no entanto não é tão direta e se baseia no fato bem conhecido de uma isometria de $S^{n}$ se estender diferenciavelmente a uma isometria de $\mathbb{R}^{n+1}-\{0\}$. Ilustraremos então esse fato, dando uma breve demonstração para o caso que nos interessa, $n=3$. Seja, portanto, $f: S^{3} \rightarrow S^{3}$ uma isometria que preserva orientação. Definamos então a seguinte função $F: \mathbb{R}^{4}-\{0\} \rightarrow \mathbb{R}^{4}-\{0\}$, dada por

$$
F(x)=\|x\| f\left(\frac{x}{\|x\|}\right) .
$$

Afirmamos então que $F$ é uma isometria. De fato, se denotarmos $\pi(x)=\frac{x}{\|x\|}$, é fácil calcular que

$$
\mathrm{d} F_{x}(v)=\frac{\langle x, v\rangle}{\|x\|} f \circ \pi(x)+\|x\| \mathrm{d} f \circ \mathrm{d} \pi_{x}(v) .
$$

Agora, repare que, se $v_{1}, v_{2} \in T_{x} \mathbb{R}^{4}, f(\pi(x))$ e $\mathrm{d} f\left(\mathrm{~d} \pi_{x}\left(v_{i}\right)\right), i=1,2$, são ortogonais. Note também que $f \circ \pi(x) \in S^{3}$. Portanto,

$$
\begin{aligned}
\left\langle\mathrm{d} F_{x}\left(v_{1}\right), \mathrm{d} F_{x}\left(v_{2}\right)\right\rangle & =\frac{\left\langle x, v_{1}\right\rangle\left\langle x, v_{2}\right\rangle}{\|x\|^{2}}+\|x\|^{2}\left\langle\mathrm{~d} f \circ \mathrm{d} \pi_{x}\left(v_{1}\right), \mathrm{d} f \circ \mathrm{d} \pi_{x}\left(v_{2}\right)\right\rangle \\
& =\frac{\left\langle x, v_{1}\right\rangle\left\langle x, v_{2}\right\rangle}{\|x\|^{2}}+\|x\|^{2}\left\langle\mathrm{~d} \pi_{x}\left(v_{1}\right), \mathrm{d} \pi_{x}\left(v_{2}\right)\right\rangle
\end{aligned}
$$

pois $f$ é isometria. Agora, um simples cálculo nos mostra que

$$
\mathrm{d} \pi_{x}(v)=-\frac{\langle x, v\rangle}{\|x\|^{3}} x+\frac{v}{\|x\|} .
$$

Substituindo então na equação anterior e refazendo os cálculos, obtemos

$$
\left\langle\mathrm{d} F_{x}\left(v_{1}\right), \mathrm{d} F_{x}\left(v_{2}\right)\right\rangle=\left\langle v_{1}, v_{2}\right\rangle
$$

e portanto $F$ é uma isometria (observe que aqui foi utilizado fortemente o fato de que a métrica definida na distribuição de $S^{3}$ é a restrição da métrica euclidiana do 
$\left.\mathbb{R}^{4}\right)$. Além disso, pela construção, $F$ preserva $S^{3}$. Temos então que necessariamente $F$, e portanto $f$ também, é restrição de uma aplicação de $O(4, \mathbb{R})$. Observe agora que, se $x \in S^{3}$ e $v \in \mathcal{D}_{x}$ tal que $\|v\|=1$, segue da construção de $\mathcal{D}$ que $\langle x, v\rangle=0$ e portanto $\{x, v\}$ é base unitária de $\mathbb{C}^{2}$. Mas, como $f$ é isometria, segue que

$$
F_{(v)}=\mathrm{d} F_{(v)}=\mathrm{d} f_{(v)} \in \mathcal{D}_{f_{(x)}=F_{(x)}}
$$

e isso significa que $\left\langle F_{(x)}, F_{(v)}\right\rangle=0$ e portanto $F$ leva a base unitária $\{x, v\}$ na base unitária $\left\{F_{(x)}, F_{(v)}\right\}$. Segue então que $F$, e portanto $f$, é restrição de uma aplicação de $U(2)$. Logo, Isom ${ }^{+}\left(S^{3}\right)=U(2)$. É evidente que $U(2)$ age transitivamente em $S^{3}$. Para notar que $U(2)$ age transitivamente em $B\left(S^{3}\right)$, basta observar que o grupo de isotropia de $p_{0}=(1,0)$ é dado por

$$
\operatorname{Isom}_{p_{0}}^{+}\left(S^{3}\right)=\left\{\left[\begin{array}{ll}
1 & 0 \\
0 & z
\end{array}\right]:\|z\|=1\right\} \cong S^{1}
$$

e que tal conjunto opera transitivamente em $\pi^{-1}\left(p_{0}\right)$.

Repare por fim que $S^{3} \cong \frac{U(2)}{S^{1}} \cong S U(2)$.

(3) $Q^{3}$

De forma análoga ao feito para $S^{3}$, mostra-se que $\operatorname{Isom}^{+}\left(Q^{3}\right)=U(1,1)$, que $\operatorname{Isom}_{p}^{+}\left(Q^{3}\right)$ $\cong S^{1}$ e que $\operatorname{Isom}^{+}\left(Q^{3}\right)$ atua transitivamente em $B\left(Q^{3}\right)$. Conclui-se ainda que $Q^{3} \cong U(1,1) / S^{1} \cong S U(1,1)$.

\subsection{Teoremas de classificação}

Observação: A partir desse ponto, trataremos os casos em que $f$ preserva e os que $f$ não preserva orientação simultaneamente, utilizando sempre o primeiro sinal (o de cima) para o caso em que $f$ preserva orientação e o outro sinal para o caso em $f$ inverte a mesma.

Sejam então $f: M \rightarrow N$, isometria local, $\left(\theta^{i}, \omega\right), i=1,2,3$, e $\left(\tilde{\theta}^{i}, \widetilde{\omega}\right)$ formas fundamentais e forma de conexão sub-Riemanniana de $M$ e $N$, respectivamente. Considere então 
$\tilde{f}: B(M) \rightarrow B(N)$ dada por $\tilde{f}(x)=\mathrm{d} f(x)$. Então, é evidente que o diagrama abaixo comuta

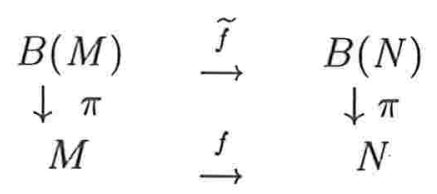

Além disso, vale a seguinte

Proposição 4.1 Se $\left(K, a, b, W_{1}, W_{2}\right)$ e $\left(\widetilde{K}, \tilde{a}, \widetilde{b}, \widetilde{W}_{1}, \widetilde{W}_{2}\right)$ são as funções definidas pelas equações de estrutura (2.9) de $M$ e $N$, respectivamente, e $f: M \rightarrow N$ é isometria local, entẫo tem-se que

$$
\begin{array}{ll}
\theta^{1}=\tilde{f}^{*} \widetilde{\theta}^{1} & a= \pm \tilde{a} \circ \tilde{f} \\
\theta^{2}= \pm \widetilde{f}^{*} \widetilde{\theta}^{2} & b=\tilde{b} \circ \tilde{f} \\
\theta^{3}= \pm \widetilde{f}^{*} \widetilde{\theta}^{3} & W_{1}=\widetilde{W}_{1} \circ \tilde{f} \\
\omega= \pm \widetilde{f}^{*} \widetilde{\omega} & W_{2}= \pm \widetilde{W}_{2} \circ \tilde{f} \\
& K=\widetilde{K} \circ \widetilde{f}
\end{array}
$$

Prova: Note primeiramente que

$$
\begin{aligned}
\left(\tilde{f}^{*} \widetilde{\theta}^{1}\right)_{v}(X) & =\tilde{\theta}_{\widetilde{f}(v)}^{1}(\mathrm{~d} \tilde{f}(X)) \\
& =\langle\mathrm{d} \pi \circ \mathrm{d} \tilde{f}(X), \tilde{f}(v)\rangle \\
& =\langle\mathrm{d} f \circ \mathrm{d} \pi(X), \mathrm{d} f(v)\rangle \\
& =\langle\mathrm{d} \pi(X), v\rangle \\
& =\theta_{v}^{1}(X) \\
\left(\tilde{f}^{*} \widetilde{\theta}^{2}\right)_{v}(X) & =\widetilde{\theta}_{\widetilde{f}(v)}^{2}(\mathrm{~d} \tilde{f}(X)) \\
& =\langle\mathrm{d} \pi \circ \mathrm{d} \tilde{f}(X), i \tilde{f}(v)\rangle \\
& =\langle\mathrm{d} f \circ \mathrm{d} \pi(X), i \mathrm{~d} f(v)\rangle \\
& =\langle\mathrm{d} f \circ \mathrm{d} \pi(X), \pm \mathrm{d} f(i v)\rangle \\
& = \pm\langle\mathrm{d} \pi(X), i v\rangle \\
& = \pm \theta_{v}^{2}(X) \\
\tilde{f}^{*} \widetilde{\theta}^{3} & =\tilde{f}^{*} \pi^{*} \tilde{\theta}=(\pi \circ \tilde{f})^{*} \tilde{\theta}
\end{aligned}
$$




$$
\begin{aligned}
& =(f \circ \pi)^{*} \tilde{\theta}=\pi^{*} f^{*} \widetilde{\theta}= \pm \pi^{*} \theta \\
& = \pm \theta^{3}
\end{aligned}
$$

Precisamos mostrar agora que $\pm \widetilde{f}^{*} \widetilde{\omega}$ é uma forma de conexão em $B(M)$.

Note antes que, por $f$ ser isometria, temos que

$$
\tilde{f} \circ R_{\theta}=R_{ \pm \theta} \circ \tilde{f}
$$

e é decorrência disso que

$$
\begin{aligned}
\mathrm{d} \tilde{f}_{v}\left(V_{v}\right) & =\mathrm{d} \tilde{f}\left(\mathrm{~d}\left(R_{t} \cdot v\right)\left(\frac{\partial}{\partial t}\right)_{0}\right) \\
& =\mathrm{d}\left(R_{ \pm t} \cdot \tilde{f}(v)\right)\left(\frac{\partial}{\partial t}\right)_{0} \\
& = \pm \mathrm{d}\left(R_{t} \cdot \tilde{f}(v)\right)\left(\frac{\partial}{\partial t}\right)_{0} \\
& = \pm \widetilde{V}_{\widetilde{f}(v)}
\end{aligned}
$$

Fica imediato então mostrar que $\pm \tilde{f}^{*} \widetilde{\omega}$ satisfaz as duas condições de forma de conexão (lembre-se que o sinal fica univocamente determinado pelo fato de $f$ preservar ou não orientação):

i) $R_{t}^{*}\left( \pm \widetilde{f}^{*} \widetilde{\omega}\right)= \pm \tilde{f}^{*}\left(R_{ \pm t}^{*} \widetilde{\omega}\right)= \pm \tilde{f}^{*} \widetilde{\omega}$

ii) $\pm \tilde{f}^{*} \tilde{\omega}\left(V_{v}\right)=\widetilde{\omega}\left( \pm \mathrm{d} \tilde{f}\left(V_{v}\right)\right)=\widetilde{\omega}\left(\tilde{V}_{\tilde{f}(v)}\right)=1$

Portanto, $\pm \tilde{f}^{*} \tilde{\omega}$ é forma de conexão. Fazendo então o "pull-back" das equações de estrutura de $B(N)$ e utilizando o fato de a diferencial e o produto exterior comutarem com o "pull back", obtemos

$$
\left\{\begin{array}{l}
\mathrm{d} \theta^{1}=\tilde{f}^{*} \widetilde{\omega} \wedge\left( \pm \theta^{2}\right)+\tilde{a} \circ \tilde{f} \theta^{1} \wedge\left( \pm \theta^{3}\right)+\widetilde{b} \circ \tilde{f}\left( \pm \theta^{2}\right) \wedge\left( \pm \theta^{3}\right) \\
\mathrm{d}\left( \pm \theta^{2}\right)=-\widetilde{f}^{*} \widetilde{\omega} \wedge \theta^{1}+\tilde{b} \circ \tilde{f} \theta^{1} \wedge\left( \pm \theta^{3}\right)-\widetilde{a} \circ \widetilde{f}\left( \pm \theta^{2}\right) \wedge\left( \pm \theta^{3}\right) \\
\mathrm{d}\left(\tilde{f}^{*} \tilde{\omega}\right)=-\widetilde{K} \circ \widetilde{f} \theta^{1} \wedge\left( \pm \theta^{2}\right)+\widetilde{W}_{1} \circ \tilde{f} \theta^{1} \wedge\left( \pm \theta^{3}\right)+\widetilde{W}_{2} \circ \widetilde{f}\left( \pm \theta^{2}\right) \wedge\left( \pm \theta^{3}\right)
\end{array}\right.
$$

ou

$$
\left\{\begin{array}{l}
\mathrm{d} \theta^{1}=\left( \pm \widetilde{f}^{*} \widetilde{\omega}\right) \wedge \theta^{2} \pm \tilde{a} \circ \tilde{f} \theta^{1} \wedge \theta^{3}+\widetilde{b} \circ \tilde{f} \theta^{2} \wedge \theta^{3} \\
\mathrm{~d} \theta^{2}=-\left( \pm \widetilde{f}^{*} \widetilde{\omega}\right) \wedge \theta^{1}+\widetilde{b} \circ \tilde{f} \theta^{1} \wedge \theta^{3}-( \pm \widetilde{a} \circ \widetilde{f}) \theta^{2} \wedge \theta^{3} \\
\mathrm{~d}\left( \pm \widetilde{f}^{*} \widetilde{\omega}\right)=-\widetilde{K} \circ \widetilde{f} \theta^{1} \wedge \theta^{2}+\widetilde{W}_{1} \circ \widetilde{f} \theta^{1} \wedge \theta^{3} \pm \widetilde{W}_{2} \circ \widetilde{f} \theta^{2} \wedge \theta^{3}
\end{array}\right.
$$


Segue então, da unicidade da forma de conexão sub-Riemanniana, que

$$
\begin{array}{ll}
\omega= \pm \tilde{f}^{*} \tilde{\omega} & W_{1}=\widetilde{W}_{1} \circ \tilde{f} \\
a= \pm \tilde{a} \circ \tilde{f} & W_{2}= \pm \widetilde{W}_{2} \circ \tilde{f} \\
b=\tilde{b} \circ \tilde{f} & K=\widetilde{K} \circ \widetilde{f}
\end{array}
$$

Sabemos desde a seção (2.3) que o tensor $\tau=\tau^{1} \otimes E_{1}+\tau^{2} \otimes E_{2}$ e a função $K$, bem como o campo $W=W_{1} E_{1}+W_{2} E_{2}$ são projetáveis sobre $M$. Disso decorre então que podemos falar nas funções, bem definidas em $M$,

$$
\begin{aligned}
K(m) & =K(v) \\
\lambda(m) & =\sqrt{-\operatorname{det}\left(\tau_{m}\right)}=\left(a_{(v)}^{2}+b_{(v)}^{2}\right)^{\frac{1}{2}} \\
\sigma(m) & =\left\|W_{(m)}\right\|=\left(W_{1(v)}^{2}+W_{2(v)}^{2}\right)^{\frac{1}{2}}, \quad v \in \pi^{-1}(m),
\end{aligned}
$$

onde abusamos da notação, denotando pelas mesmas letras as projeções de $\tau, W$ e $K$.

Com essa notação, segue da Proposição 4.1 o seguinte

Corolário 4.1 Se $f: M \rightarrow M$ é isometria local e $K, \lambda$ e $\sigma$ são as funções definidas acima, então $K=K \circ f, \lambda=\lambda \circ f$ e $\sigma=\sigma \circ f$.

Prova: Basta notar que, se $\tilde{f}$ é a induzida por $f$ no fibrado $B(M)$, então

$$
\lambda(m)=\left(a_{(v)}^{2}+b_{(v)}^{2}\right)^{\frac{1}{2}}=\left(\left( \pm a \circ \tilde{f}_{(v)}\right)^{2}+b \circ \tilde{f}_{(v)}^{2}\right)^{\frac{1}{2}}=\lambda(f(m)) .
$$

A demonstração para $K$ e $\sigma$ é análoga.

Vejamos agora o fato de a derivada covariante sub-Riemanniana, definida na seção 3.1, comutar com as isometrias.

Proposição 4.2 Seja $f: M \rightarrow M$ uma isometria local, $\nabla$ a derivada covariante subRiemanniana e $X, Y$ campos em $M$. Então

$$
f_{*}\left(\nabla_{Y} X\right)=\nabla_{f_{*} Y} f_{*} X
$$


Prova: Seja $\tilde{f}$ a induzida por $f$ a $B(M)$. Se $c: U \rightarrow S(U)$ é uma secção diferenciável, basta então que mostremos que (4.1) vale para $X=c, i . c, \xi$. Seja então $\tilde{c}$, secção definida em $f(U)$, dada por $\tilde{c}=f_{*} c$. Note que $f_{*}(i c)= \pm i f_{*} c= \pm i \widetilde{c}$, de acordo com que $f$ preserve ou não a orientação em $\mathcal{D}$. Teremos então que

$$
\begin{aligned}
\nabla_{f_{*} Y} f_{*} c & =\nabla_{f_{*} Y} \tilde{c}=\tilde{c}^{*} \omega\left(f_{*}(Y)\right) i \tilde{c} \\
& =(\widetilde{c} \circ f)^{*} \omega(Y) i \tilde{c} \\
& =(\tilde{f} \circ c)^{*} \omega(Y) i \widetilde{c} \\
& =c^{*} \tilde{f}^{*} \omega(Y) i \tilde{c} \\
& =c^{*}( \pm \omega)(Y) i \tilde{c} \\
& =c^{*} \omega(Y)( \pm i \tilde{c}) \\
& =c^{*} \omega(Y)\left(f_{*}(i c)\right) \\
& =f_{*}\left(c^{*} \omega(Y) i c\right) \\
& =f_{*}\left(\nabla_{Y} c\right) \\
\nabla_{f_{*}(Y)} f_{*}(i c) & = \pm \nabla_{f^{*}}(Y) i \widetilde{c}=\mp \tilde{c}^{*} \omega\left(f_{*}(Y)\right) \tilde{c} \\
& =\mp\left( \pm c^{*} \omega(Y)\right) \tilde{c} \\
& =-c^{*} \omega(Y) f_{*}(c) \\
& =f_{*}\left(-c^{*} \omega(Y) c\right) \\
& =f_{*}\left(\nabla_{Y} i c\right) \\
\nabla_{f_{*}(Y)} f_{*}(\xi) & =\nabla_{f_{*}(Y)}( \pm \xi)=0=f_{*}(0)=f_{*}\left(\nabla_{Y} \xi\right)
\end{aligned}
$$

Corolário 4.2 Se $f: M \rightarrow M$ é uma isometria local e $\tau$ é o tensor torsão sobre $M$, definido na seção 2.3, então $f_{*} \circ \tau= \pm \tau \circ f_{*}$.

Prova: Basta observar que

$$
T\left(f_{*} X, f_{*} Y\right)=\nabla_{f_{*} X} f_{*} Y-\nabla_{f_{*}} f_{*} X-\left[f_{*} X, f_{*} Y\right]
$$




$$
\begin{aligned}
& =f_{*}\left(\nabla_{X} Y-\nabla_{Y} X-[X, Y]\right) \\
& =f_{*}(T(X, Y))
\end{aligned}
$$

Portanto,

$$
\begin{aligned}
f_{*}(\tau(X)) & =f_{*}(T(X, \xi)) \\
& =T\left(f_{*} X, f_{*} \xi\right) \\
& =T\left(f_{*} X, \pm \xi\right) \\
& = \pm T\left(f_{*} X, \xi\right) \\
& = \pm \tau\left(f_{*} X\right)
\end{aligned}
$$

Temos ainda o seguinte corolário da Proposição 4.1:

Corolário 4.3 Se $f: M \rightarrow M$ é isometria local e $W^{\#}$ é o campo em $M$, como definido na seção 2.3, então $f_{*} W^{\#}=W^{\#} \circ f$.

Prova: Basta reparar que $W^{\#}=W_{1} \circ c \otimes c+W_{2} \circ c \otimes i c$ e que, pela Proposição 4.1,

$$
\begin{aligned}
f_{*}\left(W^{\#}\right) & =W_{1} \circ c \otimes f_{*} c \pm W_{2} \circ c \otimes i f_{*} c \\
& =W_{1} \circ \tilde{f} \circ c \otimes \tilde{c}+W_{2} \circ \tilde{f} \circ c \otimes i \tilde{c} \\
& =W_{1} \circ \tilde{c} \otimes \tilde{c}+W_{2} \circ \tilde{c} \otimes i \tilde{c} \\
& =W^{\#} \circ f
\end{aligned}
$$

Seja então $(M, \mathcal{D},\langle\rangle$,$) uma variedade sub-Riemanniana de contato e G$ um grupo de isometrias que age transitivamente sobre $M$. Sabemos então que $\lambda=\lambda(m), \sigma$ e $K=K(m)$ são constantes em $M$. Se $\lambda \neq 0$, o fato de $\tau$ ser simétrico nos garante uma direção "privilegiada" em $\mathcal{D}$ (a do auto-vetor associado ao auto-valor $\lambda$ ). Seja então uma seção $c: M \rightarrow B(M)$ de auto-vetores associados a $\lambda$. Fazendo o "pull-back" pela seção $c$ 
e escrevendo as equações de estrutura, teremos

$$
\begin{aligned}
\mathrm{d} \theta^{1} & =\omega \wedge \theta^{2}+\lambda \theta^{1} \wedge \theta \\
\mathrm{d} \theta^{2} & =-\omega \wedge \theta^{1}-\lambda \theta^{2} \wedge \theta \\
\mathrm{d} \theta & =2 \theta^{1} \wedge \theta^{2} \\
\mathrm{~d} \omega & =-K \theta^{1} \wedge \theta^{2}+W_{1} \circ c \theta^{1} \wedge \theta+W_{2} \circ c \theta^{2} \circ \theta
\end{aligned}
$$

onde já sabemos que $\lambda$ e $K$ são constantes.

Aqui denotamos com as mesmas letras, $\theta^{1}, \theta^{2}, \theta$ e $\omega$, os "pull-backs" das respectivas formas de $B(M)$. Note que $\left(\theta^{1}, \theta^{2}, \theta\right)$ aqui é a base dual a $(c, i c, \xi)$.

Queremos mostrar agora que $W_{1} \circ c$ e $W_{2} \circ c$ são constantes. Para tanto, note que $\mathrm{d} f$ leva, necessariamente, auto-vetores de $\tau$ em auto-vetores de $\tau$, pois, se $X$ é auto-vetor associado ao auto-valor $\lambda$ de $\tau$, note que

$$
\tau(\mathrm{d} f(X))= \pm \mathrm{d} f(\tau(X))= \pm \mathrm{d} f(\lambda X)= \pm \lambda \mathrm{d} f(X)
$$

Portanto, $\mathrm{d} f(X)$ é auto-vetor associado ao auto-valor $\lambda$ (ou $-\lambda$ se $f$ não preserva orientação). É conseqüência imediata que

i) $\mathrm{d} f\left(c_{(m)}\right) \in\left\{c_{f(m)},-c_{f(m)}, i c_{f(m)},-i c_{f(m)}\right\}$.

Sabemos já, no entanto, que $W_{f(m)}^{\#}=\mathrm{d} f\left(W_{m}^{\#}\right)$. Temos então que

ii) $W_{1} \circ c_{(f(m))} c_{f(m)}+W_{2} \circ c_{(f(m))} i c_{(f(m))}=\mathrm{d} f\left(W_{1} \circ c_{(m)} c_{(m)}+W_{2} \circ c_{(m)} i c_{(m)}\right)$

$$
=W_{1} \circ c_{(m)} \mathrm{d} f\left(c_{(m)}\right) \pm W_{2} \circ c_{(m)} i \mathrm{~d} f(c(m)) \text {. }
$$

i) e ii) implicam que

$$
W_{i} \circ c_{(f(m))} \in A=\left\{ \pm W_{1} \circ c_{(m)}, \pm W_{2} \circ c_{(m)}\right\}, \quad i=1,2 .
$$

Fazendo então $f$ percorrer o grupo $G$ de isometrias, o fato de a ação de $G$ ser transitiva nos garante que as funções

$$
W_{i} \circ c: M \rightarrow \mathbb{R}, \quad i=1,2
$$


vão assumir valores no conjunto discreto $A$. Se supusermos então $M$ conexo, da continuidade de $W_{i} \circ c$, temos que $W_{i} \circ c, i=1,2$, são funções constantes.

Acabamos de demonstrar então a seguinte proposição:

Proposição 4.3 Seja $(M, \mathcal{D},\langle\rangle$,$) uma variedade sub-Riemanniana homogênea de di-$ mensão 3, conexa, com forma de conexão sub-Riemanniana $\omega$, com $\tau \neq 0$. Então existe, a menos de se tomar o recobrimento duplo de $M$, uma seção $c: M \rightarrow B(M)$, em relação à qual as equações de estrutura se escrevem

$$
\begin{aligned}
\mathrm{d} \theta^{1} & =\omega \wedge \theta^{2}+\lambda \theta^{1} \wedge \theta \\
\mathrm{d} \theta^{2} & =-\omega \wedge \theta^{1}-\lambda \theta^{2} \wedge \theta \\
\mathrm{d} \theta & =2 \theta^{1} \wedge \theta^{2} \\
\mathrm{~d} \omega & =-K \theta^{1} \wedge \theta^{2}+W_{1} \theta^{1} \wedge \theta+W_{2} \theta^{2} \wedge \theta
\end{aligned}
$$

onde $K, \lambda, W_{1}$ e $W_{2}$ são constantes.

Observe que as 1-formas acima são formas em $M$ : são as de $B(M)$ "puxadas" a $M$ pela seção $c$. Note ainda que $\left(\theta^{1}, \theta^{2}, \theta\right)$ é base dual a $(c, i c, \xi)$ que passaremos a denotar por $\left(e_{1}, e_{2}, e_{3}\right)$ e que se tomarmos a seção $-c$, os sinais de $W_{1}$ e $W_{2}$ mudam simultaneamente. Podemos então supor $c$ tal que $W_{1} \geq 0$.

Note agora que, do fato de

$$
\left\{\mathrm{d} f\left(c_{(m)}\right), \mathrm{d} f\left(i c_{(m)}\right)\right\} \subset\left\{ \pm c_{f(m)}, \pm i c_{f(m)}\right\}
$$

e de que $\left\langle\mathrm{d} f\left(c_{(m)}\right), \mathrm{d} f\left(i c_{(m)}\right)\right\rangle=\left\langle c_{(m)}, i c_{(m)}\right\rangle=0$, temos que, dado $x_{0} \in M$, o grupo de isotropia de $x_{0}, G_{x_{0}}$, possui não mais que oito elementos, sendo portanto discreto. Isso se dá devido ao fato de que uma isometria (Riemanniana) fica bem determinada pelos valores de d $f$ em alguma base de $T_{x_{o}} M$. É fácil notar então que $\pi: G \rightarrow G / G_{x_{0}} \cong M$ é aplicação de recobrimento (para se construir as vizinhanças fundamentais basta utilizar o fato de $\pi$ ser homeomorfismo local, $G_{x_{0}}$ ser finito e $G$ ser Hausdorff). Portanto, se supusermos $M$ simplesmente conexa, a componente conexa $\mathcal{I}$ de $G$ que contém a identidade é um grupo 
de Lie que age transitivamente sobre $M$, é difeomorfa a $M$ e é tal que, se $f \in \mathcal{I}$, então $f^{*} \theta^{i}=\theta^{i}, i=1,2,3, \ldots, \mathrm{d} f\left(c_{m}\right)=c_{f_{(m)}}$ e d $f\left(i c_{(m)}\right)=i c_{f(m)}$. De fato, se $\gamma:[0,1] \rightarrow \mathcal{I}$ é contínua tal que $\gamma(0)=I d$ e $\gamma(1)=f$, então

$$
\begin{aligned}
& \alpha^{i}:[0,1] \rightarrow\{-1,0,1\} \\
& \alpha_{(t)}^{i}=\theta^{i}\left(\mathrm{~d}\left(\gamma_{(t)}\right)_{m}\left(c_{(m)}\right)\right)
\end{aligned}
$$

é contínua e, portanto, constante. Logo,

$$
\theta^{i}\left(\mathrm{~d} f\left(c_{m}\right)\right)=\alpha_{(1)}^{i}=\alpha_{(0)}^{i}=\theta^{i}\left(c_{(m)}\right)= \begin{cases}1 & , \text { se } i=1 \\ 0 & , \text { se } i=2 .\end{cases}
$$

Portanto, $\mathrm{d} f\left(c_{m}\right)=c_{f(m)}$. Analogamente, $\mathrm{d} f\left(i c_{m}\right)=i c_{f(m)}$. Portanto, $f$ preserva orientação e d $f\left(\xi_{m}\right)=\xi_{f(m)}$. É conseqüência imediata que $f^{*} \theta^{i}=\theta^{i}, i=1,2$, e $f^{*} \theta=\theta$. Seja então

$$
\omega=\Gamma_{1} \theta^{1}+\Gamma_{2} \theta^{2}+\Gamma \theta
$$

Fazendo então $a=\lambda=$ cte. $\neq 0$ e $b=0$ nas primeiras equações de Bianchi (2.10), obtemos facilmente que

$$
\begin{aligned}
& W_{1}=2 \lambda \Gamma_{1} \\
& W_{2}=-2 \lambda \Gamma_{2}
\end{aligned}
$$

Note então que, como $W_{1}, W_{2}$ e $\lambda$ são constantes, segue que $\Gamma_{1}$ e $\Gamma_{2}$ também o são. Da segunda equação de Bianchi, obtemos

$$
W_{1} \Gamma_{1}+W_{2} \Gamma_{2}=0
$$

e utilizando (4.2) e (4.3), obtemos

$$
\frac{W_{1}^{2}-W_{2}^{2}}{2 \lambda}=0
$$

e portanto $W_{1}= \pm W_{2}$. Observe então que, como $\Gamma_{1}$ e $\Gamma_{2}$ são constantes,

$$
\mathrm{d} \omega=\Gamma_{1} \mathrm{~d} \theta^{1}+\Gamma_{2} \mathrm{~d} \theta^{2}+\Gamma \mathrm{d} \theta^{3}+\mathrm{d} \Gamma \wedge \theta
$$

Por outro lado,

$$
\mathrm{d} \omega=-K \theta^{1} \wedge \theta^{2}+W_{1} \theta^{1} \wedge \theta+W_{2} \theta^{2} \wedge \theta
$$


e, calculando então $\mathrm{d} \omega\left(e_{1}, e_{2}\right)$ em ambas as equações, obteremos que

$$
\begin{aligned}
-K & =\Gamma_{1}^{2}+\Gamma_{2}^{2}+2 \Gamma \\
\Gamma & =-\frac{1}{2}\left(K+\Gamma_{1}^{2}+\Gamma_{2}^{2}\right)=-\frac{1}{2}\left(K+\frac{W_{1}^{2}+W_{2}^{2}}{4 \lambda^{2}}\right) \quad(\lambda \neq 0) .
\end{aligned}
$$

Portanto, $\Gamma$ é também constante, então $\mathrm{d} \Gamma=0 \mathrm{e}$

$$
\begin{aligned}
& W_{1}=\mathrm{d} \omega\left(e_{1}, e_{3}\right)=\Gamma_{1} \lambda+\Gamma_{2} \Gamma=\frac{W_{1}}{2}+\Gamma_{2} \Gamma \\
& W_{2}=\mathrm{d} \omega\left(e_{2}, e_{3}\right)=-\Gamma_{1} \Gamma-\Gamma_{2} \lambda=\frac{W_{2}}{2}-\Gamma_{1} \Gamma
\end{aligned}
$$

Então,

$$
\begin{aligned}
& W_{1}=2 \Gamma_{2} \Gamma \\
& W_{2}=-2 \Gamma_{1} \Gamma
\end{aligned}
$$

Note ainda que

$$
\Gamma_{1} \lambda=\Gamma_{2} \Gamma
$$

Podemos resumir as relações acima obtidas assim:

$$
\left\{\begin{array}{l}
K=-\left(2 \Gamma+\Gamma_{1}^{2}+\Gamma_{2}^{2}\right) \\
W_{1}=2 \Gamma_{2} \Gamma=2 \lambda \Gamma_{1} \\
W_{2}=-2 \Gamma_{1} \Gamma=-2 \lambda \Gamma_{2} \\
W_{1}= \pm W_{2} .
\end{array}\right.
$$

Além disso, das primeiras equações de estrutura e do fato que $\theta^{i}\left(\left[e_{j}, e_{k}\right]\right)=-\mathrm{d} \theta^{i}\left(e_{j}, e_{k}\right)$, temos que

$$
\begin{aligned}
& {\left[e_{1}, e_{2}\right]=-\Gamma_{1} e_{1}-\Gamma_{2} e_{2}-2 e_{3}} \\
& {\left[e_{2}, e_{3}\right]=\Gamma e_{1}+\lambda e_{2}} \\
& {\left[e_{3}, e_{1}\right]=\lambda e_{1}+\Gamma e_{2}}
\end{aligned}
$$

Antes de prosseguirmos, enunciaremos o Teorema de Frobenius, fundamental para que obtenhamos os próximos resultados, cuja demonstração pode ser encontrada em [11], p.75.

Teorema 4.1 Seja $M^{n}$ uma variedade diferenciável e $\eta^{1}, \ldots, \eta^{p}, 0<\bar{p}<n$, 1-formas diferenciáveis, independentes tais que $\mathrm{d} \eta^{i}=\sum_{j=1}^{p} \alpha_{j}^{i} \wedge \eta^{j}$, para algumas 1-formas $\alpha_{j}^{i}$. Então, fixando $m \in M$, existe uma única variedade $I$, de dimensão $n-p$, conexa maximal, passando em $m$, tal que $\eta^{i}(v)=0, \forall i=1, \ldots, p, \forall v \in T I$. 
Sejam $M$ e $N$ variedades diferenciáveis e $G$ um grupo que age em $N$. Podemos então definir uma ação de $G$ em $M \times N$ dada por

$$
\begin{aligned}
G \times(M \times N) & \rightarrow M \times N \\
(g,(m, n)) & \mapsto(m,(g, n)) .
\end{aligned}
$$

Demonstraremos agora uma proposição que nos será bastante útil mais adiante.

Proposição 4.4 Sejam $M^{m}$ e $N^{n}$ duas variedades diferenciáveis, $G$ um grupo que age transitivamente em $N^{n}$ e $\mathcal{D}$ uma distribuição em $M \times N$, m-dimensional, horizontal (ou seja, $\mathrm{d} \pi_{p}: \mathcal{D}_{p} \rightarrow T_{\pi(p)} M$ é isomorfismo linear, $\forall p \in M \times N$, onde $\pi: M \times N \rightarrow M$ denota a projeção no primeiro fator) integrável e invariante pela ação de $G$ em $M \times N$, isto é, $\mathrm{d} g_{p}\left(\mathcal{D}_{p}\right)=\mathcal{D}_{g p}, \forall p \in M \times N, \forall g \in G$. Se I é uma variedade conexa, maximal, integral a $\mathcal{D}$, então $\left.\pi\right|_{I}: I \rightarrow M$ é aplicação de recobrimento.

Prova: Faremos a demonstração, provando que $\left.\pi\right|_{I}: I \rightarrow M$ possui a propriedade do levantamento único de $\operatorname{arcos}$ (ver [2] pp 383). Seja $\gamma:[0,1] \rightarrow M$, contínua e seja $x \in I$ tal que $\pi(x)=\gamma(0)$. Seja $A$ o conjunto

$$
A=\left\{t \in[0,1]: \text { existe um levantamento } \tilde{\gamma} \text { de }\left.\gamma\right|_{[0, t]} \operatorname{com} \tilde{\gamma}(0)=x\right\}
$$

$A$ é evidentemente da forma $\left[0, t_{0}\right)$ ou $\left[0, t_{0}\right]$.

$A$ é aberto: Suponha $t_{0} \in A, t_{0}<1$. Existe então $\tilde{\gamma}:\left[0, t_{0}\right] \rightarrow I$ tal que $\pi \circ \tilde{\gamma} \equiv \gamma$ e $\tilde{\gamma}(0)=x$. Note agora que o fato de $\mathrm{d} \pi_{p}\left(\mathcal{D}_{p}\right)=T_{\pi(p)} M, \forall p \in M \times N$, garante que $\left.\pi\right|_{I}$ é difeomorfismo local. Seja então $U$, vizinhança de $\widetilde{\gamma}\left(t_{0}\right)$ em $I$, tal que $\left.\pi\right|_{U}: U \rightarrow \pi(U)$ seja um difeomorfismo. É claro então que

$$
\begin{aligned}
& \widetilde{\widetilde{\gamma}}:\left[t_{0}, t_{0}+\varepsilon\right] \rightarrow I \\
& \widetilde{\widetilde{\gamma}}(t)=\left(\left.\pi\right|_{U}\right)^{-1} \circ \gamma(t),
\end{aligned}
$$

onde $\varepsilon>0$ é tal que $\gamma\left(\left[t_{0}, t_{0}+\varepsilon\right]\right) \subset \pi(U)$ e $t_{0}+\varepsilon \leq 1$, estende continuamente $\tilde{\gamma}$ ao intervalo $\left[0, t_{0}+\varepsilon\right]$ e tal extensão é ainda levantamento de $\gamma$ iniciando em $x$. Portanto, $t_{0}$ é ponto interior a $A$. 
$A$ é fechado: Suponha que $A \supset\left[0, t_{0}\right)$. Basta que mostremos que $t_{0} \in A$. Seja $\left(\gamma\left(t_{0}\right), q\right) \in$ $M \times N$. Considere a variedade integral a $\mathcal{D}, I_{q}$, conexa que passa por $\left(\gamma\left(t_{0}\right), q\right)$. Sabemos que $\left.\pi\right|_{I_{q}}$ é difeomorfismo local. Podemos utilizar então esse fato para levantarmos a curva $\left.\gamma\right|_{\left[t_{0}-\varepsilon, t_{0}+\varepsilon\right]}$ a $I_{q}$. Seja então $\tilde{\widetilde{\gamma}}:\left[t_{0}-\varepsilon, t_{0}+\varepsilon\right] \rightarrow I_{p}$ tal levantamento. Seja agora $\tilde{\gamma}:\left[0, t_{0}-\varepsilon\right] \rightarrow I$ um levantamento de $\gamma$, com $\tilde{\gamma}(0)=x$. Como, por hipótese, $G$ age transitivamente em $N$ e $\pi \circ \tilde{\gamma}\left(t_{0}-\varepsilon\right)=\pi \circ \widetilde{\widetilde{\gamma}}\left(t_{0}-\varepsilon\right)=\gamma\left(t_{0}-\varepsilon\right)$, temos que existe $g \in G$ tal que $g\left(\tilde{\widetilde{\gamma}}\left(t_{0}-\varepsilon\right)\right)=\tilde{\gamma}\left(t_{0}-\varepsilon\right)$. Note agora que o produto por um elemento de $G$ leva variedade integral em variedade integral e portanto

$$
g \cdot I_{q} \subset I
$$

desde que $g . I_{q}$ é variedade integral a $\mathcal{D}$ que passa por $\tilde{g}\left(t_{0}-\varepsilon\right) \in I$ e $I$ é maximal. Ora, segue então que a curva $g . \tilde{\widetilde{\gamma}}$ estende continuamente $\widetilde{\gamma}$ ao intervalo $\left[0, t_{0}+\varepsilon\right]$ e portanto $t_{0} \in A$.

Da conexidade de $[0,1]$ e de $A \neq \emptyset$, segue que $A=[0,1]$ e a existência do levantamento está garantida. Suponha agora $\tilde{\gamma}:[0,1] \rightarrow I$ e $\widetilde{\gamma}:[0,1] \rightarrow I$ dois levantamentos de $\gamma$ tais que $\tilde{\gamma}(0)=\tilde{\widetilde{\gamma}}(0)=x$. Seja $A=\{t \in[0,1]: \widetilde{\gamma}(t)=\tilde{\widetilde{\gamma}}(t)\}$. A continuidade de $\tilde{\gamma}$ e $\widetilde{\widetilde{\gamma}}$ nos garante que $A$ é fechado e o fato de $A$ ser aberto decorre de $\left.\pi\right|_{I}$ ser difeomorfismo local. Como $0 \in A \neq \emptyset$, segue novamente da conexidade de $[0,1]$ que $A=[0,1]$ e portanto $\tilde{\gamma} \equiv \widetilde{\widetilde{\gamma}}$

Estamos agora em condições de demonstrar o seguinte

Teorema 4.2 Se $(M, \mathcal{D},\langle\rangle$,$) e \left(N, \mathcal{D}^{\prime},\langle\rangle,\right)$ são variedades sub-Riemannianas de contato homogêneas, de dimensão 3, simplesmente conexas, que possuem os mesmos invariantes $\lambda>0, K, W_{1} \geq 0$ e $W_{2}$, definidos anteriormente, então elas são isométricas.

Prova: Sabemos já que podemos escrever $\omega=\Gamma_{1} \theta^{1}+\Gamma_{2} \theta^{2}+\Gamma \theta$ e $\widetilde{\omega}=\Gamma_{1} \widetilde{\theta}_{1}+\Gamma_{2} \widetilde{\theta}^{2}+$ $\Gamma \tilde{\theta}$, onde $\omega$ e $\tilde{\omega}$ são as formas de conexão sub-Riemannianas, "puxadas" para $M$ e $N$, respectivamente, pelas seções que diagonalizam o tensor torsão e determinam $W_{1} \geq 0$. Observe que ambas se escrevem da mesma maneira, devido ao fato de que os coeficientes 
da forma de conexão são determinados univocamente pelos invariantes $\lambda>0, K, W_{1}, W_{2}$. Observe então que as primeiras equações de estrutura se escrevem

$$
\begin{aligned}
\mathrm{d} \theta^{1} & =\Gamma_{1} \theta^{1} \wedge \theta^{2}+\lambda \theta^{1} \wedge \theta-\Gamma \theta^{2} \wedge \theta, \\
\mathrm{d} \theta^{2} & =\Gamma_{2} \theta^{1} \wedge \theta^{2}+\Gamma \theta^{1} \wedge \theta-\lambda \theta^{2} \wedge \theta, \\
\mathrm{d} \theta & =2 \theta^{1} \wedge \theta^{2}
\end{aligned}
$$

tanto em $M$, como em $N$. Considere agora a variedade produto $M \times N$ e $\pi_{1}$ e $\pi_{2}$ as respectivas projeções sobre $M$ e $N$. Considere agora em $M \times N$ as seguintes formas (identificando $\theta^{3}:=\theta$ )

$$
\eta^{i}=\pi_{1}^{*} \theta^{i}-\pi_{2}^{*} \tilde{\theta}^{i}, \quad i=1,2,3
$$

Note que

$$
\begin{aligned}
\mathrm{d} \eta^{1} & =\Gamma_{1} \Delta_{12}+\lambda \Delta_{13}-\Gamma \Delta_{23} \\
\mathrm{~d} \eta^{2} & =\Gamma_{2} \Delta_{12}+\Gamma \Delta_{13}-\lambda \Delta_{23} \\
\mathrm{~d} \eta^{3} & =2 \Delta_{12}
\end{aligned}
$$

onde

$$
\Delta_{i j}=\pi_{1}^{*}\left(\theta^{i} \wedge \theta^{j}\right)-\pi_{2}^{*}\left(\tilde{\theta}^{i} \wedge \tilde{\theta}^{j}\right), \quad 1 \leq i<j \leq 3
$$

Agora, note que

$$
\begin{aligned}
\Delta_{i j} & =\pi_{1}^{*} \theta^{i} \wedge \pi_{1}^{*} \theta^{j}-\pi_{2} \widetilde{\theta}^{i} \wedge \pi_{2}^{*} \tilde{\theta}^{j} \\
& =\pi_{1}^{*} \theta^{i} \wedge \eta^{j}-\pi_{2}^{*} \widetilde{\theta}^{j} \wedge \eta^{i}
\end{aligned}
$$

É imediata então a conclusão que

$$
\mathrm{d} \eta^{i}=\sum_{j=1}^{3} \alpha_{j}^{i} \wedge \eta^{j}
$$

para algum conjunto de 1-formas em $M \times N,\left\{\alpha_{j}^{i}\right\}_{i, j=1,2,3}$. Então, o conjunto de formas $\left\{\eta^{i}\right\}_{i=1,2,3}$ satisfaz as condições do teorema de Frobenius. É evidente pela construção que as 1-formas $\eta^{i}$ são independentes, portanto, dado $(m, n) \in M \times N$, existe uma única variedade $I$, conexa, maximal, de dimensão 3 , que passa por $(m, n)$, integral a $\left\{\eta^{i}\right\}$, isto é, $\eta^{i}(v)=0, \forall v \in T I$ (ver figura a seguir). 

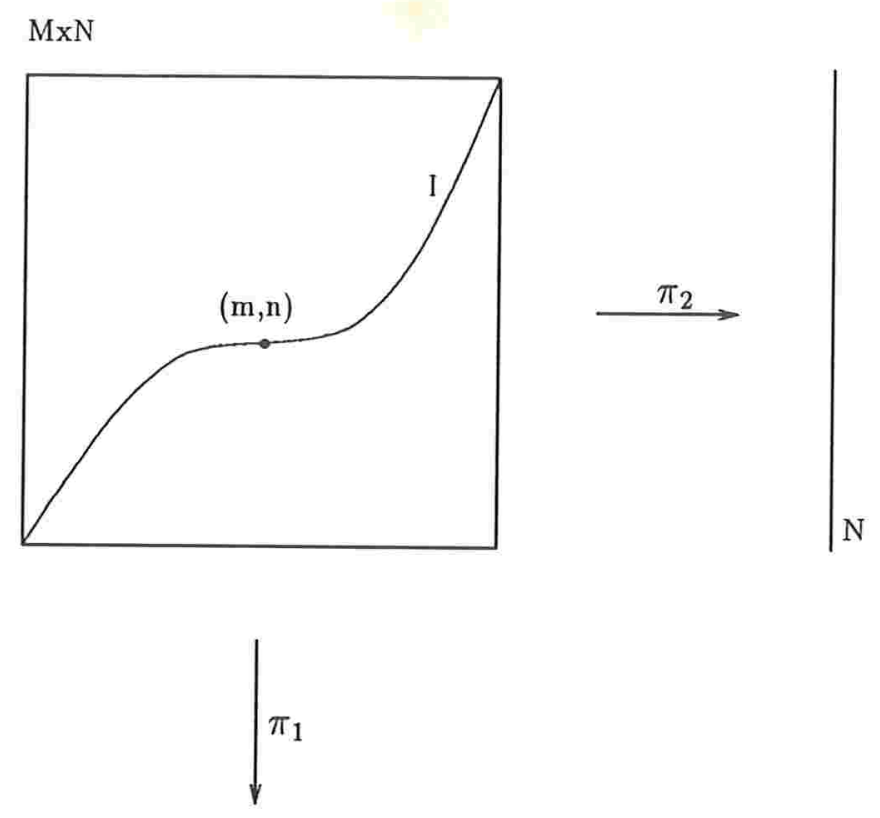

M

Sabemos ainda que $N$ possui um grupo de isometrias $\mathcal{I}$ que age transitivamente sobre $N$, tal que $f^{*} \widetilde{\theta}^{i}=\widetilde{\theta}^{i}, i=1,2,3, \forall f \in \mathcal{I}$. Observe então que, considerando a ação de $\mathcal{I}$ sobre $M \times N$,

$$
\begin{aligned}
f^{*}\left(\eta^{i}\right) & =f^{*}\left(\pi_{1}^{*} \theta^{i}-\pi_{2}^{*} \widetilde{\theta}^{i}\right) \\
& =\left(\pi_{1} \circ f\right)^{*} \theta^{i}-\left(\pi_{2} \circ f\right)^{*} \widetilde{\theta}^{i} \\
& =\pi_{1}^{*} \theta^{i}-\left(f \circ \pi_{2}\right)^{*} \widetilde{\theta}^{i} \\
& =\pi_{1}^{*} \theta^{i}-\pi_{2}^{*} f^{*} \widetilde{\theta}^{i} \\
& =\pi_{1}^{*} \theta^{i}-\pi_{2}^{*} \tilde{\theta}^{i} \\
& =\eta^{i}
\end{aligned}
$$

Note agora que essa última igualdade equivale a dizer que a distribuição $\Lambda$ dada por $\Lambda_{p}=\bigcap_{i=1}^{3} \operatorname{ker}\left(\eta_{p}^{i}\right)$ é invariante pela ação de $\mathcal{I}$ e que o fato de as $\eta^{i}$ satisfazerem (4.5) nos diz que $\Lambda$ é integrável. Além disso, note que $\left.\mathrm{d} \pi_{1(p)}\right|_{\Lambda_{p}}$ é isomorfismo linear. De fato, como $\operatorname{dim} \Lambda_{p}=3$, basta mostrar que $\left.\mathrm{d} \pi_{1(p)}\right|_{\Lambda_{p}}$ é injetora. Para isso, note que, se $\mathrm{d} \pi_{1}(v)=0, v \in \Lambda_{p}$, segue da definição de $\Lambda$ que $\eta^{i}(v)=0, i=1,2,3$, e portanto $\widetilde{\theta}^{i}\left(\mathrm{~d} \pi_{2}(v)\right)=\theta^{i}\left(\mathrm{~d} \pi_{1}(v)\right)=\theta^{i}(0)=0, \forall i=1,2,3$, portanto, $\mathrm{d} \pi_{2}(v)=0$ e então $v=0$. 
Estamos então exatamente nas condições da Proposição 4.3. Temos então que $\left(I,\left.\pi_{1}\right|_{I}\right)$ é recobrimento de $M$. Mas, como $M$ é simplesmente conexa e $I$ é conexa, segue que $\left.\pi_{1}\right|_{I}$ é homeomorfismo e, como $\left.\pi_{1}\right|_{I}$ é difeomorfismo local, segue que $\left.\pi_{1}\right|_{I}: I \rightarrow M$ é um difeormorfismo. Por simetria, temos que $\left.\pi_{2}\right|_{I} \rightarrow N$ é difeomorfismo. Então, $h: M \rightarrow N$ dada por

$$
h=\left.\pi_{2}\right|_{I} \circ\left(\left.\pi_{1}\right|_{I}\right)^{-1}=\left.\pi_{2} \circ \pi_{1}\right|_{I} ^{-1}
$$

é a isometria procurada. De fato, $h$ é difeomorfismo e

$$
\begin{aligned}
h^{*} \widetilde{\theta}^{i} & =\left(\pi_{2} \circ\left(\left.\pi_{1}\right|_{I}\right)^{-1}\right)^{*} \widetilde{\theta}^{i} \\
& =\left(\left.\pi_{1}\right|_{I} ^{-1}\right)^{*} \pi_{2}^{*} \widetilde{\theta}^{i} \\
& =\left(\left.\pi_{1}\right|_{I} ^{-1}\right)^{*} \pi_{1}^{*} \theta^{i} \\
& =\left(\pi_{1} \circ\left(\left.\pi_{1}\right|_{I}\right)^{-1}\right)^{*} \theta^{i} \\
& =I_{d}^{*} \theta^{i} \\
& =\theta^{i},
\end{aligned}
$$

onde utilizamos o fato de que, restrito a $I$,

$$
\pi_{1}^{*} \theta^{i}-\pi_{2}^{*} \tilde{\theta}^{i}=\eta^{i} \equiv 0
$$

Segue de imediato então que d $h$ leva $\mathcal{D}$ em $\mathcal{D}^{\prime}$ e que $h$ preserva a métrica. Portanto, $h$ é isometria.

Antes de darmos a demonstração dos próximos teoremas, enunciaremos dois resultados já conhecidos da geometria Riemanniana que se farão necessários nas demonstrações posteriores.

Proposição 4.5 Seja $M$ uma variedade Riemanniana. Se $M$ admite um grupo transitivo de isometrias, então $M$ é completa.

Proposição 4.6 Seja $M$ uma variedade Riemanniana completa e $f: M \rightarrow N$ um difeomorfismo local sobre uma variedade Riemanniana com a seguinte propriedade:

$$
\forall v \in T M, \quad\|\mathrm{~d} f(v)\| \geq\|v\| .
$$

Então $f$ é aplicação de recobrimento. 
Teorema 4.3 Seja $(M, \mathcal{D},\langle\rangle$,$) uma variedade sub-Riemanniana de contato de di-$ mensão 3, simplesmente conexa, completa (no sentido Riemanniano), com $\tau=0, W_{1}=$ $W_{2}=0$ e curvatura constante igual a $0(K$ ou $-K, K>0)$. Então $M$ é isométrica a $H^{3}$ $\left(S_{r}^{3}\right.$ ou $\widetilde{Q}_{r}^{3}$, respectivamente, onde $\left.r=(4 / K)^{1 / 2}\right)$.

Prova: Observe inicialmente que $\tau=0$ implica necessariamente que $W_{1}=W_{2}=0$ (basta fazer $a=b=0$ nas identidades de Bianchi (2.10)). Denotemos por $N$ a segunda variedade em questão $\left(H^{3}, S^{3}\right.$ ou $\left.\widetilde{Q}^{3}\right)$. Considere as formas $\left(\theta^{i}, \omega\right)$ em $B(M)$ e $\left(\tilde{\theta}^{i}, \widetilde{\omega}\right)$ em $B(N)$. Definamos $\eta^{i}, i=0,1,2,3$, em $B(M) \times B(N)$ como no teorema anterior

$$
\begin{aligned}
\eta^{0} & =\pi_{1}^{*} \omega-\pi_{2}^{*} \widetilde{\omega} \\
\eta^{i} & =\pi_{1}^{*} \theta^{i}-\pi_{2}^{*} \widetilde{\theta}^{i} .
\end{aligned}
$$

Da mesma forma como feito anteriormente, $\left(\eta^{i}\right)_{i=0,1,2,3}$, satisfarão às hipóteses do teorema de Frobenius, i.e.,

$$
\mathrm{d} \eta^{i}=\sum_{j=0}^{n} \alpha_{j}^{i} \eta^{j}
$$

para algumas 1-formas $\left\{\alpha_{j}^{i}\right\}_{i, j=0,1,2,3}$ em $B(M) \times B(N)$.

Sejam então $\left(v_{1}, v_{2}\right) \in B(M) \times B(N)$ e $I$ a única variedade integral a $\left\{\eta^{i}\right\}$, conexa, maximal e que passa por $\left(v_{1}, v_{2}\right)$, dada pelo teorema de Frobenius. De forma análoga ao feito anteriormente, concluímos que $\left.\pi_{1}\right|_{I}: I \rightarrow B(M)$ e $\left.\pi_{2}\right|_{I}: I \rightarrow B(N)$ são difeomorfismos locais e que $\left(I,\left.\pi_{1}\right|_{I}\right)$ é recobrimento de $B(M)$. Observe no entanto que, para obtermos esse último resultado, precisamos utilizar o fato de que $N\left(H^{3}, S^{3}\right.$ ou $\widetilde{Q}^{3}$, conforme o caso) possui um grupo de isometrias que atua transitivamente no fibrado $B(N)$, isto é, que dados $v_{1}, v_{2} \in T B(N)$, existe uma isometria $f$ tal que $\mathrm{d} f\left(v_{1}\right)=v_{2}$, fato que foi demonstrado na seção 4.2 .

Considere agora a seguinte ação de $S^{1}$ sobre $B(M) \times B(N)$ :

$$
\begin{aligned}
S^{1} \times(B(M) \times B(N)) & \rightarrow B(M) \times B(N) \\
R_{t}(v, w) & =\left(R_{t} v, R_{t} w\right) .
\end{aligned}
$$

Note que $\pi_{i} \circ R_{t}=R_{t} \circ \pi_{i}, i=1,2$. Queremos mostrar agora que essa ação de $S^{1}$ está bem definida em $I$, em outras palavras, que $I$ é invariante por esta ação. Para isso, basta 
mostrarmos que, para $(v, w) \in I$,

$$
\left.\frac{\mathrm{d}}{\mathrm{d} t}\left(R_{t}(v, w)\right)\right|_{t=0} \in T_{(v, w)} I
$$

Mas, pela construção de $I$, isso é equivalente a mostrar que

$$
\eta^{i}\left(\left.\frac{\mathrm{d}}{\mathrm{d} t}\left(R_{t}(v, w)\right)\right|_{t=0}\right)=0, i=0,1,2,3 .
$$

Mas,

$$
\begin{aligned}
\eta^{0}\left(\left.\frac{\mathrm{d}}{\mathrm{d} t}\left(R_{t}(v, w)\right)\right|_{t=0}\right) & =\left(\pi_{1}^{*} \omega-\pi_{2}^{*} \widetilde{\omega}\right)\left(\mathrm{d}\left(R_{t}(v, w)\right)\left(\frac{\partial}{\partial t}\right)_{0}\right) \\
& =\omega\left(\mathrm{d}\left(\pi_{1} \circ R_{t}(v, w)\right)\left(\frac{\partial}{\partial t}\right)_{0}\right)-\widetilde{\omega}\left(\mathrm{d}\left(\pi_{2} \circ R_{t}(v, w)\right)\left(\frac{\partial}{\partial t}\right)_{0}\right) \\
& =\omega\left(\mathrm{d}\left(R_{t} \circ \pi_{1}(v, w)\right)\left(\frac{\partial}{\partial t}\right)_{0}\right)-\widetilde{\omega}\left(\mathrm{d}\left(R_{t} \circ \pi_{2}(v, w)\right)\left(\frac{\partial}{\partial t}\right)_{0}\right) \\
& =\omega\left(\mathrm{d}\left(R_{t} \cdot v\right)\left(\frac{\partial}{\partial t}\right)_{0}\right)-\widetilde{\omega}\left(\mathrm{d}\left(R_{t} \cdot w\right)\left(\frac{\partial}{\partial t}\right)_{0}\right) \\
& =\omega\left(V_{v}\right)-\widetilde{\omega}\left(V_{w}\right) \\
& =1-1 \\
& =0
\end{aligned}
$$

e analogamente,

$$
\eta^{i}\left(\left.\frac{\mathrm{d}}{\mathrm{d} t}\left(R_{t}(v, w)\right)\right|_{t=0}\right)=\theta^{i}\left(V_{v}\right)-\theta^{i}\left(V_{w}\right)=0-0=0, \quad i=1,2,3 .
$$

Se denotarmos então por $\pi$, a projeção de $I$ sobre $I / S^{\mathbf{1}}$, e percebermos que $\left.\pi \circ \pi_{1}\right|_{I}: I \rightarrow M$, passa ao quociente (isso é conseqüência direta de $\pi_{1} \circ R_{t}=R_{t} \circ \pi_{1}$ ), obteremos o seguinte diagrama comutativo

$$
\begin{array}{ccc}
I & \stackrel{\left.\pi_{1}\right|_{I}}{\longrightarrow} & B(M) \\
\downarrow \pi & \widetilde{\left.\pi_{1}\right|_{I}} & \downarrow \pi \\
I / S^{1} & \stackrel{M}{\longrightarrow} &
\end{array}
$$

onde $\widetilde{\left.\pi_{1}\right|_{I}}$ é evidentemente a induzida por $\left.\pi_{1}\right|_{I}$. Agora, como $\left(I,\left.\pi_{1}\right|_{I}\right)$ é aplicação de recobrimento, a equivariância de $\pi_{1}$, isto é, o fato de $\pi_{1} \circ R_{t}=R_{t} \circ \pi_{1}$, nos garante que $\left(I / S^{1}, \widetilde{\left.\pi_{1}\right|_{I}}\right)$ recobre $M$. Portanto, $\widetilde{\left.\pi_{1}\right|_{I}}$ é homeomorfismo (pois $M$ é simplesmente conexo). A diferenciabilidade de $\widetilde{\left.\pi_{1}\right|_{I}}$ é decorrência direta da comutatividade do diagrama (e é claro, 
da existência de seções diferenciáveis $c: I / S^{1} \rightarrow I$ que, por sua vez, é decorrência da "estrutura quociente"). Note ainda que $\widetilde{\left.\pi_{1}\right|_{I}}$ é difeomorfismo local, pois $\operatorname{dim}\left(I / S^{1}\right)=3$ e as diferenciais de $\left.\pi_{1}\right|_{I}$ e $\pi: B(M) \rightarrow M$ possuem posto máximo. Concluímos portanto que $\widetilde{\left.\pi_{1}\right|_{I}}$ é um difeomorfismo.

É claro que, por outro lado, também possuímos o diagrama comutativo

$$
\begin{array}{ccc}
I & \stackrel{\left.\pi_{2}\right|_{I}}{\longrightarrow} & B(N) \\
\downarrow \pi & \widetilde{\downarrow} & \downarrow \pi \\
I / S^{1} & \widetilde{\left.\pi_{2}\right|_{I}} & N
\end{array}
$$

Da mesma forma, $\widetilde{\left.\pi_{2}\right|_{I}}$ é difeomorfismo local. A função que procuramos então, vai ser dada por

$$
\begin{aligned}
& \tilde{f}: \quad M \rightarrow N \\
& \tilde{f}=\left.\tilde{\pi}_{2}\right|_{I} \circ\left(\left.\tilde{\pi}_{1}\right|_{I}\right)^{-1} .
\end{aligned}
$$

Observe que $\tilde{f}$ é um difeomorfismo local. Queremos mostrar que $\tilde{f}$ é, localmente, isometria.

De fato, sabemos já que $\left.\pi_{1}\right|_{I} \rightarrow B(M)$ e $\left.\pi_{2}\right|_{I}: I \rightarrow B(N)$ são difeomorfismos locais e que $\left.\pi_{1}\right|_{I}$ é aplicação de recobrimento. Seja então $W$, aberto de $I$, tal que $\left.\pi_{i}\right|_{W}, i=$ 1,2 , sejam difeomorfismos sobre suas imagens. Considere então $f: \pi_{1}(W) \subset B(M) \rightarrow$ $\pi_{2}(W) \subset B(N)$, dada por $f \equiv \pi_{2} \circ\left(\left.\pi_{1}\right|_{W}\right)^{-1}$. Denotemos $U=\pi_{1}(W)$ e $V=\pi(U) \in M$. Podemos supor $U$ suficientemente pequeno tal que $\left.\widetilde{f}\right|_{V}$ seja também um difeomorfismo. Note agora que $f$ é tal que comuta o diagrama abaixo:

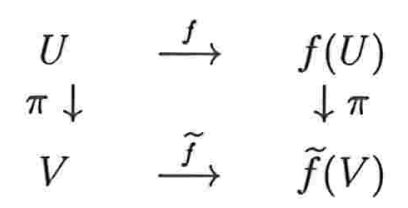

De fato, a comutatividade desse diagrama decorre da comutatividade (já sabida) dos diagramas abaixo:

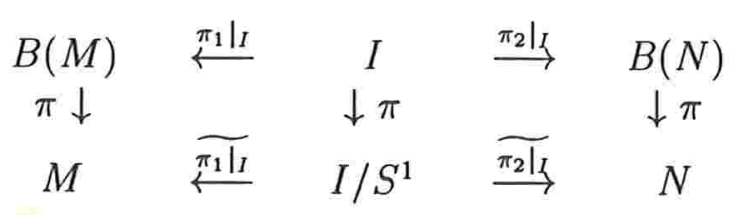


Seja então $c: V \rightarrow U$ uma seção diferenciável e considere $\bar{c}: \tilde{f}(V) \rightarrow f(U)$ a seção definida em $\tilde{f}(V) \subset N$ que comuta o gráfico

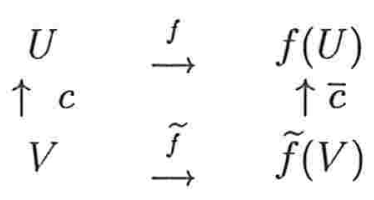

Sejam também $\left(c^{*} \theta^{1}, c^{*} \theta^{2}, c^{*} \theta^{3}\right)$ e $\left(\bar{c}^{*} \theta^{1}, \bar{c}^{*} \theta^{2}, \bar{c}^{*} \theta^{3}\right)$ bases duais a $(c, i c, \xi)$ e $(\bar{c}, i \bar{c}, \bar{\xi})$. Então,

$$
\begin{aligned}
\tilde{f}^{*}\left(\bar{c}^{*} \tilde{\theta}^{i}\right) & =c^{*} f^{*} \widetilde{\theta}^{i} \\
& =c^{*}\left(\left.\pi_{2}\right|_{I} \circ\left(\left.\pi_{1}\right|_{W}\right)^{-1}\right)^{*} \tilde{\theta}^{i} \\
& =c^{*}\left(\left(\left.\pi_{1}\right|_{W}\right)^{-1}\right)^{*}\left(\left.\pi_{2}\right|_{I}\right)^{*} \widetilde{\theta}^{i} \\
& =c^{*}\left(\left(\left.\pi_{1}\right|_{W}\right)^{-1}\right)^{*}\left(\left.\pi_{1}\right|_{I}\right)^{*} \theta^{i} \\
& =c^{*} \theta^{i}
\end{aligned}
$$

Portanto, $\tilde{f}: M \rightarrow N$ é, localmente, isometria e como por hipótese, $M$ é completa, segue da proposição 4.6 que $(M, \widetilde{f})$ é recobrimento de $N$, mas $N$ é sabidamente simplesmente conexa. Portanto, como $M$ é conexa, $\tilde{f}$ é difeomorfismo.

Observe que, devido à proposição 4.5, podemos substituir a hipótese "completa" por "homogênea" no enunciado do teorema 4.3.

\subsection{Classificação das variedades sub-Riemannianas de contato homogêneas de dimensão 3}

Seja agora $G$ um grupo de Lie simplesmente conexo e também uma variedade subRiemanniana de contato homogênea com invariantes $K, \lambda>0$. Sabemos então, pela proposição 4.3 e pelas relações obtidas posteriormente, que existe uma seção $e_{1}$ tal que as equações de estrutura da álgebra de Lie se escrevem

$$
\begin{aligned}
& {\left[e_{1}, e_{2}\right]=-\frac{W_{1}}{2 \lambda} e_{1}+\frac{W_{2}}{2 \lambda} e_{2}-2 e_{3}} \\
& {\left[e_{2}, e_{3}\right]=\Gamma e_{1}+\lambda e_{2}} \\
& {\left[e_{3}, e_{1}\right]=\lambda e_{1}+\Gamma e_{2}}
\end{aligned}
$$


onde $0 \leq W_{1}= \pm W_{2}, K=-2 \Gamma-\left(-\frac{W_{1}}{2 \lambda}\right)^{2}-\left(\frac{W_{2}}{2 \lambda}\right)^{2}=-2\left(\Gamma+\left(\frac{W_{1}}{2 \lambda}\right)^{2}\right)$ e, se $W_{1} \neq 0$, então $\Gamma=-\operatorname{sgn}\left(W_{2}\right) \lambda$.

Supondo então $W_{1}=W_{2}=0$. Teremos então que

$$
\begin{aligned}
& {\left[e_{1}, e_{2}\right]=-2 e_{3}} \\
& {\left[e_{2}, e_{3}\right]=-\frac{K}{2} e_{1}+\lambda e_{2}} \\
& {\left[e_{3}, e_{1}\right]=\lambda e_{1}-\frac{K}{2} e_{2}}
\end{aligned}
$$

Não é difícil verificar então que, de acordo com os valores de $K$ e $\lambda>0$, recairemos nos grupos de Lie clássicos, que descreveremos abaixo. Nessa descrição, definiremos o grupo, forneceremos uma base $\left(f_{1}, f_{2}, f_{3}\right)$ para sua álgebra de Lie e diremos quem deverão ser $\left(e_{1}, e_{2}, e_{3}\right)$ para que se recaia nas equações de estrutura (4.6).

(1) $K>2 \lambda$

$$
G \cong S U(2)=\left\{A \in G L(2, \mathbb{C}): A \cdot A^{*}=I \quad \text { e } \quad \operatorname{det} A=1\right\},
$$

onde $A^{*}=\bar{A}^{t}$.

$$
\begin{array}{cl}
f_{1}=\left[\begin{array}{cc}
i & 0 \\
0 & -i
\end{array}\right] & e_{1}=\frac{1}{2}\left(\sqrt{\frac{K}{2}+\lambda} f_{2}-\sqrt{\frac{K}{2}-\lambda f_{1}}\right) \\
f_{2}=\left[\begin{array}{cc}
0 & 1 \\
-1 & 0
\end{array}\right] & e_{2}=\frac{1}{2}\left(\sqrt{\frac{K}{2}+\lambda f_{2}}+\sqrt{\frac{K}{2}-\lambda f_{1}}\right) \\
f_{3}=\left[\begin{array}{cc}
0 & i \\
i & 0
\end{array}\right] & e_{3}=\frac{1}{4}\left(\sqrt{K^{2}-4 \lambda^{2}}\right) f_{3}
\end{array}
$$

(2) $K=2 \lambda$

$G=\widetilde{E(2)}$, onde o til indica o recobrimento universal e

$$
E(2)=\left\{\left[\begin{array}{lll}
1 & 0 & 0 \\
x & & \\
y & A
\end{array}\right], \text { onde } x, y \in \mathbb{R} \quad \text { e } \quad A \in S O(2, \mathbb{R})\right\}
$$




$$
\begin{array}{ccc}
f_{1}=\left[\begin{array}{lll}
0 & 0 & 0 \\
1 & 0 & 0 \\
0 & 0 & 0
\end{array}\right] & e_{1}=\sqrt{2 \lambda} f_{2}-f_{1} \\
f_{2}=\left[\begin{array}{lll}
0 & 0 & 0 \\
0 & 0 & 1 \\
0 & -1 & 0
\end{array}\right] & e_{2}=\sqrt{2 \lambda} f_{2}+f_{1} \\
f_{3}=\left[\begin{array}{lll}
0 & 0 & 0 \\
0 & 0 & 0 \\
1 & 0 & 0
\end{array}\right] & e_{3}=\sqrt{2 \lambda} f_{3}
\end{array}
$$

(3) $-2 \lambda<K<2 \lambda$,

$G \cong \widetilde{S U(1,1)}$

$$
S U(1,1)=\left\{A \in G L(2, \mathbb{C}): A \mathcal{I} A^{*}=\mathcal{I} \quad \text { e } \quad \operatorname{det} A=1\right\},
$$

onde $\mathcal{I}=\left[\begin{array}{cc}1 & 0 \\ 0 & -1\end{array}\right]$.

$$
\begin{array}{cl}
f_{1}=\left[\begin{array}{cc}
0 & i \\
-i & 0
\end{array}\right] & e_{1}=\frac{1}{2}\left(\sqrt{\lambda+\frac{K}{2}} f_{2}-\sqrt{\lambda-\frac{K}{2}} f_{1}\right) \\
f_{2}=\left[\begin{array}{cc}
i & 0 \\
0 & -i
\end{array}\right] & e_{2}=\frac{1}{2}\left(\sqrt{\lambda+\frac{K}{2}} f_{2}+\sqrt{\lambda-\frac{K}{2}} f_{1}\right) \\
f_{3}=\left[\begin{array}{ll}
0 & 1 \\
1 & 0
\end{array}\right] & e_{3}=\frac{1}{4}\left(\sqrt{4 \lambda^{2}-K^{2}}\right) f_{3}
\end{array}
$$

(4) $K=-2 \lambda$

$$
\begin{gathered}
G \cong E(1,1)=\left\{\left[\begin{array}{lll}
1 & 0 & 0 \\
x & \\
y & A(t)
\end{array}\right]: t, x, y \in \mathbb{R} \quad \text { e } A(t)=\left[\begin{array}{cc}
\cosh t & \operatorname{senh} t \\
\operatorname{senh} t & \cosh t
\end{array}\right]\right\} \\
f_{1}=\left[\begin{array}{lll}
0 & 0 & 0 \\
0 & 0 & 1 \\
0 & 1 & 0
\end{array}\right] \quad e_{1}=f_{2}-\sqrt{2 \lambda} f_{1}
\end{gathered}
$$




$$
\begin{array}{ll}
f_{2}=\left[\begin{array}{lll}
0 & 0 & 0 \\
1 & 0 & 0 \\
0 & 0 & 0
\end{array}\right] & e_{2}=f_{2}-\sqrt{2 \lambda} f_{1} \\
f_{3}=\left[\begin{array}{lll}
0 & 0 & 0 \\
0 & 0 & 0 \\
1 & 0 & 0
\end{array}\right] & e_{3}=\sqrt{2 \lambda} f_{3}
\end{array}
$$

(5) $K<-2 \lambda$

$$
\begin{gathered}
G \cong \widetilde{S U}(1,1),\left(f_{1}, f_{2}, f_{3}\right) \text { como definido em }(3) \mathrm{e} \\
e_{1}=\frac{1}{2}\left(\sqrt{-\left(\lambda+\frac{K}{2}\right)} f_{1}-\sqrt{\lambda-\frac{K}{2}} f_{3}\right) \\
e_{2}=\frac{1}{2}\left(\sqrt{-\left(\lambda+\frac{K}{2}\right)} f_{1}+\sqrt{\lambda-\frac{K}{2}} f_{3}\right) \\
e_{3}=\frac{1}{4} \sqrt{K^{2}-4 \lambda^{2}} f_{2}
\end{gathered}
$$

Analisadas então todas as possibilidads para $W_{1}=0$, passaremos a ver o caso em que $W_{1} \neq 0$. Deixaremos indicado abaixo como foi feita a determinação do grupo e da base $\left(e_{1}, e_{2}, e_{3}\right)$, para o caso em que $W_{2}=W_{1}$. No caso $W_{2}=-W_{1}$, a determinação é análoga.

Suponha $W_{2}=W_{1}>0$. Teremos então que $\Gamma=-\lambda$ e portanto $K=2 \lambda-2\left(\frac{W_{1}}{2 \lambda}\right)^{2}$ e teremos necessariamente que $K<2 \lambda$ e $\frac{W_{1}}{2 \lambda}=\sqrt{\lambda-\frac{K}{2}}$. As equações de estrutura ficam então

$$
\begin{aligned}
& {\left[e_{1}, e_{2}\right]=-\sqrt{\lambda-\frac{K}{2}} e_{1}+\sqrt{\lambda-\frac{K}{2}} e_{2}-2 e_{3}} \\
& {\left[e_{2}, e_{3}\right]=-\lambda e_{1}+\lambda e_{2}} \\
& {\left[e_{3}, e_{1}\right]=\lambda e_{1}-\lambda e_{2}}
\end{aligned}
$$

Se fizermos então

$$
g_{1}=e_{2}-e_{1}, \quad g_{2}=-e_{2}, \quad g_{3}=e_{3}
$$

obteremos

$$
\left[g_{1}, g_{2}\right]=\sqrt{\lambda-\frac{K}{2}} g_{1}-2 g_{3}
$$




$$
\begin{aligned}
& {\left[g_{2}, g_{3}\right]=-\lambda g_{1}} \\
& {\left[g_{3}, g_{1}\right]=0}
\end{aligned}
$$

Observe então que $\left\{g_{1}, g_{3}\right\}$ é base de uma sub-álgebra abeliana que corresponderá a um subgrupo também abeliano $H$ de $G$. Se supusermos ainda $H$ simplesmente conexo, teremos necessariamente que $H \cong \mathbb{R}^{2}$. Seja então $S \cong \mathbb{R}$ o subgrupo correspondente à sub-álgebra gerada por $\left\{g_{2}\right\}$. Se $h$ e $s$ denotam as álgebras de Lie de $H$ e $S$, note que as duas primeiras igualdades acima definem uma aplicação $\sigma: s \rightarrow g l(h)$ dada por $\sigma(X)(Y)=[Y, X]$, onde $X \in s$ e $Y \in h$. A essa aplicação corresponderá uma única $\widetilde{\sigma}: S \cong \mathbb{R} \rightarrow G L(H)$ tal que o diagrama abaixo comuta.

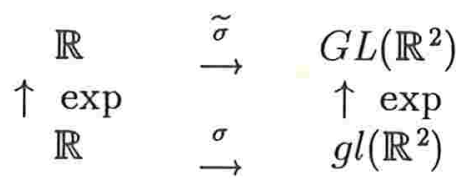

A aplicação exp $: \mathbb{R} \rightarrow \mathbb{R}$ se trata da aplicação identidade (lembre-se que a exponencial aqui é a de grupos de Lie e que o grupo considerado é $(\mathbb{R},+)$ ). Portanto, $\widetilde{\sigma}$ ficará determinada por

$$
\tilde{\sigma}(t)=\exp \left(\sigma\left(t g_{2}\right)\right)=e^{t \cdot\left[\begin{array}{cc}
\sqrt{\lambda-K / 2} & \lambda \\
-2 & 0
\end{array}\right]}
$$

e nosso grupo $G$ então será dado pelo produto semi-direto de $\mathbb{R} \times \mathbb{R}^{2}$, relativo à aplicação $\tilde{\sigma}$.

Devemos então determinar $e^{A}$, onde $A=\left[\begin{array}{ll}a & b \\ c & 0\end{array}\right]$, com $a, b, c$ diferentes de zero. Calculando então as raízes do polinômio característico, obteremos

$$
\lambda_{1}=\frac{a+\sqrt{\Delta}}{2}, \quad \lambda_{2}=\frac{a-\sqrt{\Delta}}{2}
$$

onde $\Delta=a^{2}+4 b c$ e, se $\Delta \neq 0$, então $A$ é diagonalizável sobre $\mathbb{C}$. Uma multiplicação simples nos mostra que, nesse caso,

$$
A=T\left[\begin{array}{cc}
\lambda_{1} & 0 \\
0 & \lambda_{2}
\end{array}\right] T^{-1}
$$

onde $T$ é a matriz dada por

$$
T=\left[\begin{array}{cc}
-b & -b \\
\lambda_{2} & \lambda_{1}
\end{array}\right]
$$


e, da propriedade de exponencial de matrizes, temos que

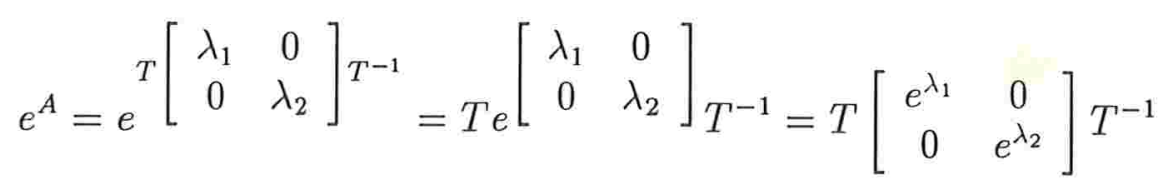

e fazendo os produtos na última expressão, obteremos que

$$
e^{A}=e^{\frac{a}{2}}\left\{\frac{\operatorname{senh}\left(\frac{\sqrt{\Delta}}{2}\right)}{\left(\frac{\sqrt{\Delta}}{2}\right)}\left[\begin{array}{cc}
a / 2 & b \\
c & -a / 2
\end{array}\right]+\cosh \frac{\sqrt{\Delta}}{2} I\right\}
$$

e note que, no caso em que $\Delta<0$,

$$
\begin{aligned}
& \frac{\operatorname{senh}\left(\frac{\sqrt{\Delta}}{2}\right)}{\left(\frac{\sqrt{\Delta}}{2}\right)}=\frac{\operatorname{sen}\left(\frac{\sqrt{-\Delta}}{2}\right)}{\left(\frac{\sqrt{-\Delta}}{2}\right)} \\
& \cosh \left(\frac{\sqrt{\Delta}}{2}\right)=\cos \left(\frac{\sqrt{-\Delta}}{2}\right)
\end{aligned}
$$

Resta-nos somente analisar o caso em que $\Delta=0$. Nesse caso, se tomarmos $T=$ $\left[\begin{array}{cc}-b & -b \\ 0 & \frac{a}{2}\end{array}\right]$, teremos que

$$
A=T D T^{-1}, \quad \text { onde } \quad D=\frac{a}{2}\left[\begin{array}{ll}
1 & 0 \\
1 & 1
\end{array}\right],
$$

cuja exponencial é simples de se calcular pelo desenvolvimento em série

$$
e^{D}=\sum_{n=0}^{\infty} \frac{D^{n}}{n !}=e^{a / 2}\left[\begin{array}{cc}
1 & 0 \\
\frac{a}{2} & 1
\end{array}\right]
$$

Portanto, teremos que

$$
\begin{aligned}
e^{A} & =T e^{D} T^{-1} \\
& =e^{a / 2}\left\{\left[\begin{array}{cc}
\frac{a}{2} & b \\
c & -\frac{a}{2}
\end{array}\right]+I\right\} .
\end{aligned}
$$

Resumindo então, obtivemos

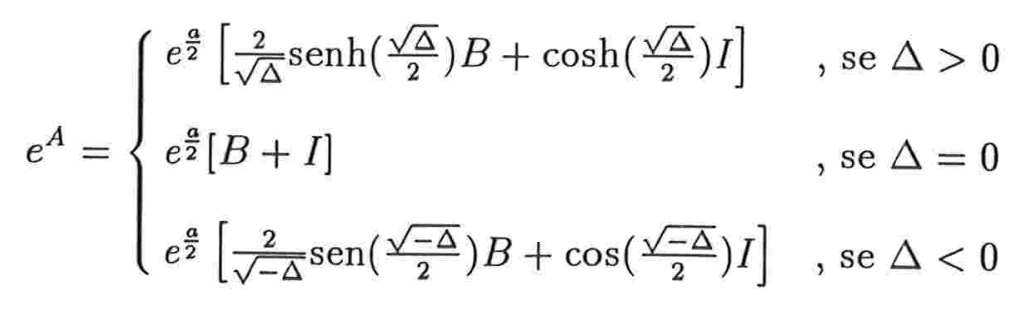


onde $A=\left[\begin{array}{ll}a & b \\ c & 0\end{array}\right], B=\left[\begin{array}{cc}a / 2 & b \\ c & -a / 2\end{array}\right]$ e $\Delta=a^{2}+4 b c$

Voltando então à determinação de $\tilde{\sigma}$, fazendo $a=t \sqrt{\lambda-\frac{K}{2}}, b=\lambda t$ e $c=-2 t$, teremos que

$$
\tilde{\sigma}(t)= \begin{cases}e^{\alpha t}\left[\frac{\operatorname{senh}(\beta t)}{\beta} B+\cosh (\beta t) I\right] & , \text { se } K<-14 \lambda, \\ e^{\alpha t}[t B+I] & , \text { se } K=-14 \lambda, \\ e^{\alpha t}\left[\frac{\operatorname{sen}\left(\beta^{\prime} t\right)}{\beta^{\prime}} B+\cos \left(\beta^{\prime} t\right) I\right] & , \text { se }-14 \lambda<K<2 \lambda,\end{cases}
$$

onde $\alpha=\frac{1}{2} \sqrt{\lambda-\frac{K}{2}}, \beta=\frac{1}{2 \sqrt{2}} \sqrt{-K-14 \lambda}, \beta^{\prime}=\frac{1}{2 \sqrt{2}} \sqrt{K+14 \lambda}$ e $B=\left[\begin{array}{cc}\alpha & \lambda \\ -2 & -\alpha\end{array}\right]$.

É fácil verificar que õ é injetora. Poderemos então utilizar a seguinte representação (fiel) para o nosso produto semi-direto:

$$
G_{1} \cong\left\{\left[\begin{array}{ccc}
0 & 0 & 0 \\
x & \\
y & & \tilde{\sigma}(t)
\end{array}\right]: x, y, t \in \mathbb{R}\right\}
$$

É fácil verificar agora que, em qualquer caso,

$$
g_{1}=-\left[\begin{array}{lll}
0 & 0 & 0 \\
1 & 0 & 0 \\
0 & 0 & 0
\end{array}\right], \quad g_{2}=-\left[\begin{array}{ccc}
0 & 0 & 0 \\
0 & 2 \alpha & \lambda \\
0 & -2 & 0
\end{array}\right], \quad g_{3}=-\left[\begin{array}{ccc}
0 & 0 & 0 \\
0 & 0 & 0 \\
1 & 0 & 0
\end{array}\right]
$$

é base da álgebra de Lie de $G_{1}$ que satisfaz (4.8) e portanto

$$
e_{1}=-\left(g_{1}+g_{2}\right), \quad e_{2}=-g_{2}, \quad e_{3}=g_{3}
$$

é base que satisfaz (4.7).

$\mathrm{O}$ caso em que $-W_{2}=W_{1}>0$, trata-se de forma semelhante e obteremos que, necessariamente, $K<-2 \lambda$ e

$$
G_{2} \cong\left\{\left[\begin{array}{ccc}
1 & 0 & 0 \\
x & \\
y & \varphi(t)
\end{array}\right]: x, y, t \in \mathbb{R}\right\}
$$

onde

$$
\begin{aligned}
& \varphi(t)=e^{\alpha t}\left[\frac{\operatorname{senh} \beta t}{\beta} B+\cosh \alpha t . I\right] \\
& \alpha=\frac{1}{2} \sqrt{-\lambda-\frac{K}{2}}, \quad \beta=\frac{1}{2 \sqrt{2}} \sqrt{14 \lambda-K}, \quad B=\left[\begin{array}{cc}
\alpha & \lambda \\
2 & -\alpha
\end{array}\right]
\end{aligned}
$$


e a base da álgebra de Lie que produzirá as equações de estruturas desejadas será

$$
e_{1}=\left[\begin{array}{ccc}
0 & 0 & 0 \\
1 & -a & -\lambda \\
0 & -2 & 0
\end{array}\right], \quad e_{2}=\left[\begin{array}{ccc}
0 & 0 & 0 \\
0 & a & \lambda \\
0 & 2 & 0
\end{array}\right], \quad e_{3}=-\left[\begin{array}{lll}
0 & 0 & 0 \\
0 & 0 & 0 \\
1 & 0 & 0
\end{array}\right]
$$

Podemos resumir então a nossa classificação na seguinte tabela:

\begin{tabular}{c|c|c|c|c} 
Grupo & $\lambda$ & $K$ & $W_{1}$ & $W_{2}$ \\
\hline$H^{3}$ & & 0 & & 0 \\
$S_{r}^{3}$ & 0 & $K=4 / r^{2}$ & 0 & 0 \\
$\widetilde{Q}_{r}^{3}$ & & $K=-4 / r^{2}$ & & \\
\hline$\frac{S U(2)}{\widetilde{E(2)}}$ & & $K>2 \lambda$ & & \\
$\frac{}{S U(1,1)}$ & $\lambda>0$ & $-2 \lambda<K<2 \lambda$ & & 0 \\
$\frac{E(1,1)}{S U(1,1)}$ & & $K=-2 \lambda$ & 0 & 0 \\
\hline$G_{1}$ & $\lambda>0$ & $K=2 \lambda-\frac{W_{1}^{2}}{2 \lambda^{2}}$ & $W_{1}>0$ & $W_{2}=W_{1}$ \\
$G_{2}$ & & $K=-2 \lambda-\frac{W_{1}^{2}}{2 \lambda^{2}}$ & $W_{1}>0$ & $W_{2}=-W_{1}$ \\
\hline
\end{tabular}

Observe que $S^{3} \cong S U(2)$ e que $Q^{3} \cong S U(1,1)$.

Note agora a seguinte curiosidade: se $M$ é o grupo de Lie, simplesmente conexo, cuja estrutura da álgebra seja dada por

$$
\begin{aligned}
& {\left[e_{1}, e_{2}\right]=\Gamma_{1} e_{1}+\Gamma_{2} e_{2}-2 e_{3}} \\
& {\left[e_{2}, e_{3}\right]=0} \\
& {\left[e_{3}, e_{1}\right]=0}
\end{aligned}
$$

é imediato que a distribuição $\mathcal{D}$ gerada por $\left(e_{1}, e_{2}\right)$ é de contato e que, se $\left(\theta^{1}, \theta^{2}, \theta^{3}\right)$ é dual a $\left(e_{1}, e_{2}, e_{3}\right)$ e tomarmos a métrica em $\mathcal{D}$ que torna $\left(e_{1}, e_{2}\right)$ base ortonormal, então, $\theta^{3}$ é forma de contato associada. Dualizando então as soluções, obteremos as primeiras equações de estrutura:

$$
\mathrm{d} \theta^{1}=-\Gamma_{1} \theta^{1} \wedge \theta^{2}
$$




$$
\begin{aligned}
& \mathrm{d} \theta^{2}=-\Gamma_{2} \theta^{1} \wedge \theta^{2} \\
& \mathrm{~d} \theta^{3}=2 \theta^{1} \wedge \theta^{2}
\end{aligned}
$$

de onde concluímos facilmente que $\tau=0$ e que a forma de conexão sub-Riemanniana será dada por

$$
\omega=-\Gamma_{1} \theta^{1}-\Gamma_{2} \theta^{2}
$$

de onde temos que

$$
\mathrm{d} \omega=\left(\Gamma_{1}^{2}+\Gamma_{2}^{2}\right) \theta^{1} \wedge \theta^{2}
$$

e portanto $K=-\left(\Gamma_{1}^{2}+\Gamma_{2}^{2}\right)$.

Portanto, se $\Gamma_{1}^{2}+\Gamma_{2}^{2} \neq 0$, segue do Teorema 4.2 que $M$ será isométrica ao recobrimento universal de $Q_{r}^{3} \cong S U(1,1)$, onde $r=\left(\frac{4}{\Gamma_{1}^{2}+\Gamma_{2}^{2}}\right)^{1 / 2}$. Perceba no entanto que $M$ não pode ser isomorfa (como grupo de Lie) a $\widehat{S U(1,1)}$, pois $S U(1,1)$ não possui sequer sub-álgebra abeliana. 


\section{Referências}

[1] CAMACHO, C. \& LINS NETO. Teoria geométrica das folheaçôes. Projeto Euclides, IMPA, Rio de Janeiro, 1979.

[2] CARMO, M.P. do. Differentiable curves and surfaces. Prentice-Hall, New Jersey, 1976.

[3] CARMO, M.P. do. Geometria Riemanniana. Projeto Euclides, IMPA, Rio de Janeiro, 1988.

[4] CHERN, S.S. \& HAMILTON, R.S. On Riemannian metrics adapted to three-dimensional contact-manifolds. Lect. Not. Math. 1111, 279-305.

[5] FALBEL, E. \& GORODSKI, C. Sub-Riemannian homogeneous spaces in dimensions 3 and 4. IME-USP (preprint), São Paulo, 1994: a ser publicada pela Geometriae Dedicata em 1996.

[6] FALBEL, E.; VELOSO, J.M. \& VERDERESI, J.A. The equivalence problem in sub-Riemannian geometry. IME-USP, São Paulo, 1993.

[7] KOBAYASHI, S. \& NOMIZU, K. Foundation of differential geometry. v.1. Interscience (Wiley), New York, 1963.

[8] SINGER, I.M. \& THORPE, J.A. Lectures notes on elementary topology and geometry. Scott, Foresman, Glenview, 1967.

[9] STRICHARTZ, R.S. Sub-Riemannian geometry. J. Diff. Geometry, 24:221-263, 1986.

[10] STRICHARTZ, R.S. Corrections to sub-Riemannian geometry. J. Diff. Geometry, 30:595-596, 1989.

[11] WARNER, F.W. Foundations of differential manifolds and Lie groups. Scott, Foresman, Glenview, 1971. 